\title{
Fit kids, healthy kids ?! : associations between overweight, insulin resistance and physical fitness in Dutch adolescents
}

Citation for published version (APA):

Slinger, J. D. (2008). Fit kids, healthy kids ?! : associations between overweight, insulin resistance and physical fitness in Dutch adolescents. [Doctoral Thesis, Maastricht University]. Universitaire Pers Maastricht. https://doi.org/10.26481/dis.20080125js

Document status and date:

Published: 01/01/2008

DOI:

10.26481/dis.20080125js

Document Version:

Publisher's PDF, also known as Version of record

Please check the document version of this publication:

- A submitted manuscript is the version of the article upon submission and before peer-review. There can be important differences between the submitted version and the official published version of record.

People interested in the research are advised to contact the author for the final version of the publication, or visit the DOI to the publisher's website.

- The final author version and the galley proof are versions of the publication after peer review.

- The final published version features the final layout of the paper including the volume, issue and page numbers.

Link to publication

\footnotetext{
General rights rights.

- You may freely distribute the URL identifying the publication in the public portal. please follow below link for the End User Agreement:

www.umlib.nl/taverne-license

Take down policy

If you believe that this document breaches copyright please contact us at:

repository@maastrichtuniversity.nl

providing details and we will investigate your claim.
}

Copyright and moral rights for the publications made accessible in the public portal are retained by the authors and/or other copyright owners and it is a condition of accessing publications that users recognise and abide by the legal requirements associated with these

- Users may download and print one copy of any publication from the public portal for the purpose of private study or research.

- You may not further distribute the material or use it for any profit-making activity or commercial gain

If the publication is distributed under the terms of Article $25 \mathrm{fa}$ of the Dutch Copyright Act, indicated by the "Taverne" license above, 


\title{
FIT KIDS, HEALTHY KIDS?!
}

\author{
Associations between overweight, \\ insulin resistance and physical fitness \\ in Dutch adolescents
}

JANTINE SLINGER 


\section{itutin}

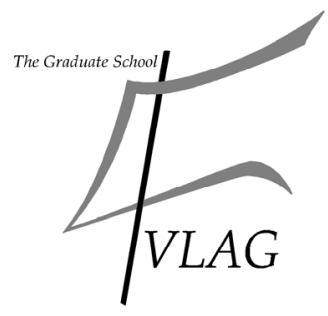

The studies presented in this thesis were performed within the Nutrition and Toxicology Research Institute Maastricht (NUTRIM) which participates in the Graduate School VLAG (Food Technology, Agrobiotechnology, Nutrition and Health Sciences), accredited by the Royal Netherlands Academy of Arts and Sciences.

Cover picture: Jantine Slinger

Cover statue: Jos Hermans

Coverdesign: Jantine Slinger \& Bilbo Schickenberg

Layout: Jantine Slinger

Printed by: Datawyse / Universitaire Pers Maastricht

( ) Jantine Slinger, Maastricht 2008

ISBN 978-90-5278-685-8 


\title{
FIT KIDS, HEALTHY KIDS?!
}

\author{
Associations between overweight, \\ insulin resistance and physical fitness \\ in Dutch adolescents
}

\author{
Proefschrift \\ ter verkrijging van de graad van doctor \\ aan de Universiteit Maastricht \\ op gezag van de Rector Magnificus, \\ Prof. mr. G.P.M.F. Mols \\ volgens het besluit van het College van Decanen, \\ in het openbaar te verdedigen \\ op vrijdag 25 januari 2008 om 12.00 uur \\ door \\ Jantine Slinger \\ geboren te Dirksland op 7 mei 1980
}

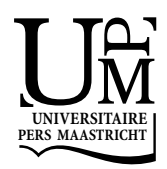




\section{Promotor}

Prof. Dr. H. Kuipers

\section{Copromotor}

Dr. E. van Breda

\section{Beoordelingscommissie}

Prof. Dr. R.A. de Bie (voorzitter)

Prof. Dr. M.A. van Baak

Dr. W.J.M. Gerver

Prof. Dr. R.A. HiraSing (Vrije Universiteit Amsterdam)

Prof. Dr. N.K. de Vries

Financial support by the Netherlands Heart Foundation and the Dutch Diabetes Research Foundation for the publication of this thesis is gratefully acknowledged. 


\section{CONTENTS}

$\begin{array}{lll}\text { Chapter } 1 & \text { General Introduction } & 7\end{array}$

Chapter 2 Insulin resistance, physical fitness, body composition and leptin concentration in 7-8 year old children

Chapter 3 Development of insulin resistance and related variables between 7 and 13 years of age: a longitudinal study

Chapter $4 \quad$ Elevated glucose concentration and physical parameters in adolescents

Chapter 5 The effect of body build and BMI on aerobic test performance in school children (10-15 years)

Chapter 6 Recent performance capacity data for Dutch adolescents

Chapter $7 \quad$ Effects of a 12 week practice based lifestyle program to counteract overweight in adolescents: an intervention study

Chapter 8 General Discussion

References

Summary

Samenvatting

Appendix

Dankwoord 


\section{CHAPTER 1}

\section{General Introduction}




\section{Background}

The prevalence of overweight and obesity is increasing worldwide (216). This increase is not only present in adults, but in children and adolescents as well (113). In the Netherlands, prevalence numbers of overweight in adults approximate $50 \%$ (201) whereas it reaches $15-20 \%$ in children and adolescents (96). These numbers are comparable to those of international studies in Western countries (180). The increased prevalence in youth is especially alarming, because also in this group overweight has been associated with certain health risks (137). For example, the prevalence of type 2 Diabetes Mellitus (type 2 DM) has been increasing in youth simultaneously with the increased prevalence of overweight. In fact, the prevalence of type $2 \mathrm{DM}$, which used to be a disease of the elderly, has been increasing in children and adolescents during recent years. While in 1982 only four percent of all pediatric diabetic cases in the US were classified as type 2, in 1994 of all diabetic cases 33\% were identified as type 2 in the group of subjects aged 0 - 19 (79). Sinha et al. (2002) pointed out that one out of every four severely obese children had an impaired glucose tolerance (172). Impaired glucose tolerance and insulin resistance are both features of type 2 DM which each independently proceed into the full blown disease. Besides body composition, decreases in physical activity and physical fitness are assumed to be associated with the increased prevalence of insulin resistance and type 2 DM in adolescents as well (26, $98,134)$. It has often been suggested that due to increasing number of hours that youngsters spend on playing computer games and watching television programs, the level of physical activity has been decreasing $(106,148)$. Such a decreased activity level is supposed to be associated with an increased prevalence of obesity on the one hand, and with a decreased level of physical fitness among the youth on the other. All together, these findings may increase the risk for insulin resistance and type 2 DM.

The increased prevalence of overweight in both children and adolescents (10-20 years of age) in combination with the global shift in the diabetic population towards a younger age formed the background for this thesis. In apparently healthy children the effects of potential risk and potential protective factors that underlie insulin resistance were investigated. This information may assist to reverse the ongoing increase in prevalence of insulin resistance in youth. In this chapter an introduction regarding the background of overweight, obesity, insulin resistance, type 2 DM mellitus and physical fitness will be given. 


\section{Overweight and obesity}

The origin of excessive fat storage can be traced back to our prehistoric ancestors. The concept of survival of the fittest led to better survival chances in individuals who stored energy efficiently during the inevitable periods of fast and famine that alternated with times of plenty (89). Nowadays, excessive fat storage is called overweight or obesity. Historically, obesity was praised in some cultures, because it indicated status and wealth (89). However, the recognition of obesity as a medical problem is only of recent date (89) and the severity of the situation concerning the upcoming obesity pandemic has just began to be fully recognized.

\section{Definition of overweight and obesity}

The World Health Organization (WHO) defined overweight and obesity as abnormal or excessive fat accumulation (216). Although from nature's point of view fat storage has a positive functional role, nowadays long-term excessive fat accumulation is classified as pathological. Body mass index (BMI) is a simple index of body weight normalized to height and is commonly used to classify overweight and obesity. It is defined as the weight in kilograms divided by the squared height $(\mathrm{kg} / \mathrm{m} 2)$. The WHO defines "overweight" as a BMI of $25 \mathrm{~kg} / \mathrm{m} 2$ or above, "obesity" as a BMI of $30 \mathrm{~kg} / \mathrm{m} 2$ or above and "severe obesity" as a BMI of $40 \mathrm{~kg} / \mathrm{m} 2$ or more. Although BMI provides an easy measure for overweight and obesity, it should only be considered and used as a rough indication because it may not accurately reflect the degree of fatness in an individual (210). For instance, when assessing body composition in people with a large muscle mass the BMI may overestimate the fat content.

Furthermore, assessing overweight and obesity in children and adolescents is not as simple as it may appear, because body mass index in childhood varies substantially with age (40). At birth the median BMI is as low as $13 \mathrm{~kg} / \mathrm{m}^{2}$, and increases to $17 \mathrm{~kg} / \mathrm{m}^{2}$ at age 1 , but decreases to $15.5 \mathrm{~kg} / \mathrm{m}^{2}$ at age 6 , then increases again to $21 \mathrm{~kg} / \mathrm{m}^{2}$ at age 20. Cole et al. (2000) established BMI cut-off points adjusted for age and gender to define pediatric overweight and obesity (41). These cut-off points are derived from the before mentioned adult cut-off points, that are related to increased health risks (141). Although BMI is an easily applicable measure, it does not give accurate information about the percentage of fat in the body of the youth either, therefore, alternative methods to evaluate body composition have been introduced. In Chapter 5 an approach using the fat mass index 
and the fat free mass index will be presented and be applied to the current population.

\section{Prevalence of overweight and obesity}

The prevalence of overweight and obesity is increasing worldwide. In 2005, there were over one billion overweight adults $\left(B M I \geq 25 \mathrm{~kg} / \mathrm{m}^{2}\right)$, from whom at least 300 million were obese $\left(B M I \geq 30 \mathrm{~kg} / \mathrm{m}^{2}\right)$ (216). Also in the Netherlands the prevalence of overweight has been increasing at an undesirable speed. In 2007, about $50 \%$ of the Dutch adult population were overweight and about $10 \%$ could be classified as obese (201). It is expected that the prevalence of obesity will increase to a higher level in the next decades (17).

Childhood obesity has been estimated to be present in one out of every ten children worldwide and over 22 million children under the age of five are servery obese (113). However, this global average includes a wide range of different prevalence numbers, with an overweight prevalence in Africa and Asia well below $10 \%$ but $20 \%$ and higher in the Americas and Europe. In comparison to the most recent surveys from the late 1990s up to 2003, it is expected that the proportion of affected school age children will be doubled by 2010 (113). In the European Union, without any preventive interventions, the number of overweight children is expected to increase by 1.3 million children each year, of which more than 300.000 will become obese (113). The increase in the prevalence of overweight and obesity is even of more concern than the prevalence of adult obesity alone because the majority of obese children remain obese as adults (137), and it has been shown that children with the highest degree of overweight also have the highest risk of becoming obese as adults (147).

In the Netherlands, some major growth studies have been executed and those studies have yielded estimates of the prevalence of overweight and obesity in all age categories. The graphic representation of this development of overweight (see figure 1a) and obesity (see figure 1b) is based on the third (1980) and fourth National Growth Study (1997), and on a recent study (2002-2003) $(91,96,195)$. Since adolescence is a sensitive period in life, between the $10^{\text {th }}$ and $20^{\text {th }}$ year of life, in which individuals start to develop their own lifestyle, in the current thesis the focus will be on the adolescent population. In 2003, the prevalence of overweight in this studied group, aged 12 - 16, was in both genders three times as high as in 1980. In 2003, the prevalence of obesity in girls was about 7 times as high as in 1980, in boys the prevalence was in 2003 about 20 times as high compared to 1980 
(91, 96, 195). Without any effective prevention strategies this increase in prevalence is expected to continue.
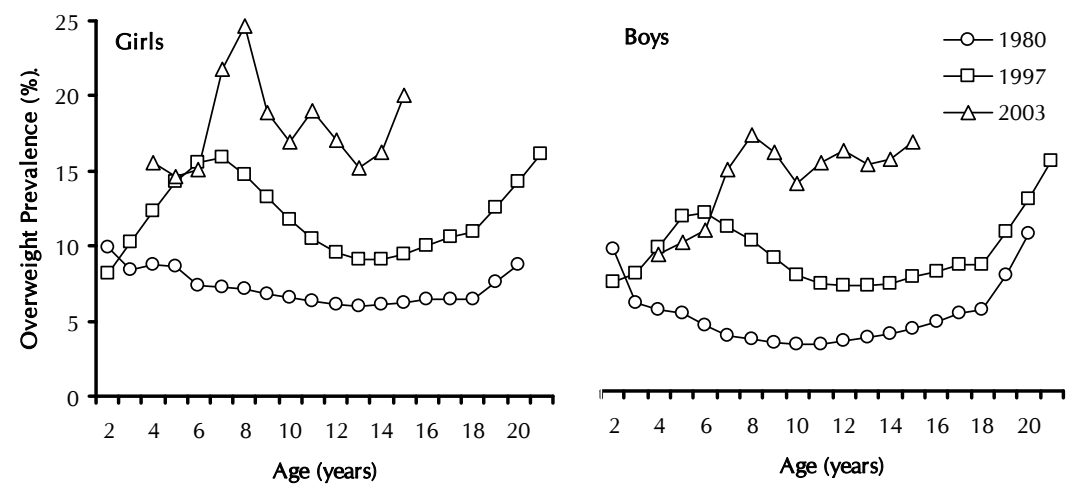

Figure 1a Prvalence of overweight in Dutch adolescents in 1980 (91), 1997 (91) and $2003(96,195)$.
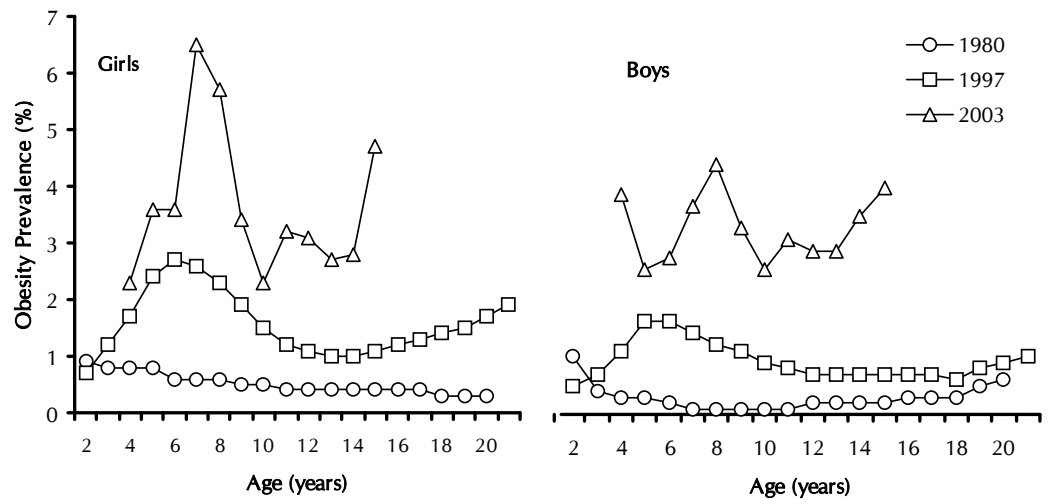

Figure 1b Prevalence of obesity in Dutch adolescents in 1980 (91), 1997 (91) and $2003(96,195)$. 


\section{Health consequences of overweight and obesity}

Overweight and obesity do not only affect the appearance of an individual, which may influence the persons self-esteem, but also has several detrimental effects on bodily functions (35). Obesity for example is the most prominent feature of the metabolic syndrome (MetS), which will be further introduced in the next paragraph. Additionally, there are other negative health consequences of obesity. Obese children have an approximately threefold higher risk for hypertension than non-obese children (177). Furthermore, obese people are more likely to develop obstructive sleep apnea syndrome (153), which is a significant problem in children and has adverse effects for growth and development (153). Another possible health problem is polycystic ovary syndrome (PCOS), which may lead to infertility $(35,53)$. In addition, overweight children and adolescents have more musculoskeletal disorders and are more likely to encounter fractures than their normal-weight counterparts $(35,43)$. In line with the latter findings, the increased mechanical joint loading that occurs in obesity may cause osteoarthritis (68), which is more prevalent in obese adolescents compared to normal weight individuals (183). All together these ailments result in a unfavorable effect on longevity and will blunt the continuous rise in life expectancy that mankind experienced since 1850 (140).

\section{Diabetes mellitus, insulin resistance and metabolic syndrome}

Diabetes Mellitus is a condition known to doctors since 1552 BC, when the Ebers papyrus referred to 'excessive urination' and 'a medicine to drive away the passing of too much urine' (89). In Hindu writings, thousands of years ago, it was stated that black ants were used to detect diabetes. Records describe a mysterious, deadly disease causing intense thirst, enormous urine output and wasting of the body. A diagnostic sign was the attraction of ants and flies to the victims' urine (89). In the $2^{\text {nd }}$ century $A D$, the Greek physician Areataeus from Capadocia gave a detailed description of diabetes:

'Diabetes is a wonderful affection, not very frequent among men, being a melting down of the flesh and limbs into urine. Its cause is of a cold and humid nature as in dropsy. The course is the common one, namely, the kidney and bladder; for the patients never stop making water, but the flow is incessant, as from the opening of aqueducts. The nature of the disease, then, is chronic, and it takes a long period to form; but the patient is short lived, if 
the constitution of the disease be completely established; for the melting is rapid' (89). $^{1}$

\section{Disease definitions}

Nowadays, the World Health Organization recognizes three main forms of diabetes: type 1, type 2 and gestational diabetes, that all have similar signs, symptoms and consequences, but have different causes and prevalence numbers (217). Type $1 \mathrm{DM}$ is usually due to autoimmune destruction of the pancreatic beta cells that produce insulin. Type 2 is characterized by insulin resistance and in some cases a progressive loss of beta cell function. Gestational diabetes is similar to type $2 \mathrm{DM}$, and involves insulin resistance. The hormone levels during pregnancy may cause insulin resistance in genetically predisposed women.

In 2006, the WHO estimated the global number of people with diabetes to be 171 million in the year 2000 and predicted the prevalence to be 366 million in the year 2030 (217). In Europe, the prevalence was 33.3 million in 2000 and is expected to increase to 48 million in 2030. Globally diabetes causes about five percent of all deaths each year and without urgent action the number of diabetes deaths is likely to increase by more than $50 \%$ during the next 10 years (216). Unfortunately there are no recent prevalence data for the Dutch population. The most recent prevalence data for the Dutch population date from 2003 and are based on registrations from general practitioners. These data suggest that more than 600.000 persons were diabetic (either type 1 or type 2) (14). In this group, $90 \%$ were diagnosed as type 2 diabetic (14). This prevalence is likely to be even higher when taking into account that it has been suggested that for every two persons diagnosed one person is still not recognized. The Dutch diabetes foundation estimated that the current prevalence in the Netherlands reaches 850.000.

In order to diagnose diabetes or one of its early stages, several methods are used. Relatively easy applicable methods are the oral glucose tolerance test and the homeostasis model assessment (HOMA) (123), whereas more sophisticated methods include the hyperinsulinemic euglycemic clamp. The most frequently used method for large scale studies is the measurement of blood glucose concentration. This can either by measured in the fasted, or during the 2-hours postprandial state. Generally used cut-off values belonging to the latter are summarized in table 1 (74).

\footnotetext{
${ }^{1}$ Translated by Francis Adams, London, 1856
} 
Table 1 Cut-off values for impaired g/ucose concentration and diabetes in fasting and 2-hour blood g/ucose concentrations

\begin{tabular}{lll}
\hline & Test \\
\hline & Fasting blood glucose & 2-hours blood glucose \\
\hline Normal & $<5.6 \mathrm{mmol} / \mathrm{l}$ & $<7.8 \mathrm{mmol} / \mathrm{l}$ \\
Impaired glucose concentration & $5.6-6.9 \mathrm{mmol} / \mathrm{l}$ & $7.8-11.0 \mathrm{mmol} / \mathrm{l}$ \\
Diabetes* & $7.0 \mathrm{mmol} / \mathrm{l}$ & $11.1 \mathrm{mmol} / \mathrm{l}$ \\
\hline
\end{tabular}

*The diagnosis of diabetes needs to be confirmed on a separate day

When insulin resistance is accompanied by other health characteristics like overweight and high blood pressure one may speak of the metabolic syndrome (MetS). This syndrome not only has been associated with type 2 DM but also with cardiovascular diseases. The MetS consists of a cluster of characteristics like elevated blood pressure, dys-lipidemia, insulin resistance and overweight and may occur in adults as well as in children and adolescents $(94,164)$. The MetS has been shown to be present in nearly half of all morbid obese children and increases proportionally with body mass index (208).

Although several intervention programs have been developed to improve overall health of overweight youngsters, only one lifestyle intervention program has been studied regarding the MetS (128). This hospital based study suggests that such a lifestyle intervention program may have beneficial effects on some of the pathological parameters of the MetS. In chapter 7 the effects of a Dutch practice based program on characteristics of the MetS in a group of overweight adolescents are presented.

Insulin resistance is one of the most 'silent' co-morbidities of the MS. It develops slowly and at first without any clinical noticeable effects. Since insulin resistance is probably 'the' most prominent risk factor for type $2 \mathrm{DM}$, in the current thesis mainly focus on insulin resistance (190). In the first stage of insulin resistance glucose concentrations can still be maintained within the physiological range. In the second stage, glucose concentration increases above the physiological range, in particular postprandial. In the next stage both increased fasting and postprandial glucose levels occur. This stage may ultimately develop into type 2 DM (190).

Age at diabetic onset

Type 2 DM was formerly known as 'diabetes for the elderly'. Pinhas-Miel et al. (1996) were the first to report about the upcoming epidemic in adolescents (145). Prior to the findings of Phinhas-Hamiel et al. (1996) it was generally accepted that type 2 DM was mainly restricted to older age groups and did not affect children. Recent incidence data suggests a global 
increase of type $2 \mathrm{DM}$ in children (146), based on data from studies in the U.S. (83, 122), Asia (120, 149), Africa (102), Australia (29) and Japan (194). European data come from Germany $(165)$, Austria $(150,165)$ and the UK (87).

A Dutch survey among Dutch pediatricians revealed that $2.4 \%$ of the diabetic children were type 2 diabetic $(14,158)$. Almost all of these patients were overweight and nearly all patients were girls. It was stated that this estimate may only reflect a fraction of the total number of patients, as it usually takes some time before diabetes is diagnosed, which results in a large number of undiagnosed patients (14). Since information about insulin resistance in the average Dutch adolescent population is lacking, the study described in Chapter 4 investigated the prevalence of an elevated blood glucose concentration in Dutch adolescents. In Chapter 3 data on the tracking of insulin resistance from 7 to 13 years of age is presented to evaluate whether insulin resistance at the age of 7 is predictive for insulin resistance at the age of 13 . Altogether this may provide more information regarding the age at which secondary prevention activities (i.e. screening) should be initiated.

The emergence of type $2 \mathrm{DM}$ in adolescents has important implications for both the health of the individual and health service resources. Compliance to type 2 DM treatment $(105,118)$ is poor, especially in children. Several studies have described a higher risk of nephropathy (186) and retinopathy (220) in type 2 diabetic patients compared to pediatric type 1 diabetic patients. This is probably because in type 1 diabetes glucose homeostasis is generally better controlled than in type 2 DM patients. Additionally, recent data show early signs of cardiovascular disease in youth with type 2 DM (85). In the only longitudinal study available 79 children were contacted 15 year after type 2 DM was diagnosed. In this group nine percent died and six percent were on dialysis (48).

The increased prevalence of insulin resistance and type 2 DM in youth in combination with its detrimental effects on health, stress the importance of effective prevention programs. For adequate prevention of type $2 \mathrm{DM}$ in children it is important to identify the key risk factors. Besides obesity some other risk factors for pediatric diabetes have been identified, such as a positive family history for diabetes, ethnic minority, puberty and female gender (8). In this thesis these and other risk factors, as well as potential protective factors will be investigated (Chapter 2, 3 and 4). 


\section{Physical fitness}

Two of the most often suggested protective factors for type 2 DM have been physical fitness and physical activity. A possible association between physical fitness and health has already been proposed in the ancient Greece society, but even in earlier civilizations (124). Physical fitness has been appreciated in different ways and therefore over time, several definitions have been proposed.

\section{Definition}

In 1979, the American Academy of Physical Education adopted the following definition:

'Physical fitness is the ability to carry out daily tasks with vigor and alertness, without undue fatigue and with ample energy to engage in leisure time pursuits and to meet the above-average physical stresses encountered in emergency situations' ${ }^{\prime}(37)$.

In 1994 the health-related fitness concept was redefined by Bouchard and Shepard (21), who proposed a scientific conceptual approach to the relationship between physical activity, physical fitness and health. The health-related fitness concept of Bouchard and Shephard (21) implies that physical activity interacts both with health as well as with disease. Healthrelated fitness refers to physical and physiological characteristics that determine the risk for premature development of diseases or health risks associated with a sedentary lifestyle. The health-related fitness of a person includes five components: 1) a morphological component (body mass for height, body composition, subcutaneous fat distribution, abdominal visceral fat, bone density and flexibility); 2) a muscular component (power or explosive strength, isometric strength, muscular endurance; 3) a motor component (agility, balance, co-ordination, speed of movement); 4) a cardio respiratory component (endurance or sub maximal exercise capacity, maximal aerobic power, heart function, lung function, blood pressure); and 5) a metabolic component (glucose tolerance, insulin sensitivity, lipid and lipoprotein metabolism, substrate oxidation characteristics) (21). In the current thesis all of these components of physical fitness will be addressed. 


\section{Fitness, physical activity and health}

The suggested, complex relationships between physical activity, fitness and health, at least in adults, are schematically depicted in figure 2 . In children and adolescents such relationships are expected to be present as well, although the quantity and quality of international research remains scarce and inconsistent (188). Cross-sectional studies have shown that in general, physically active young people are healthier in comparison with non-active young people at the level of physical and psychosocial health resources, risk factors, complaints, subjective health status and tobacco consumption (188). Additionally, physical activity early in life seems to contribute to health in adulthood (88). There is substantial evidence that physical activity tracks moderately from adolescence to adulthood, and physical activity in adolescents appears to provide long-term benefits on bone health and breast cancer risk reduction in adulthood (188). Additionally, self-esteem and mental health seem to be positively affected by physical activity (88). Finally, it has been suggested that physical activity is negatively associated with the MetS (104).

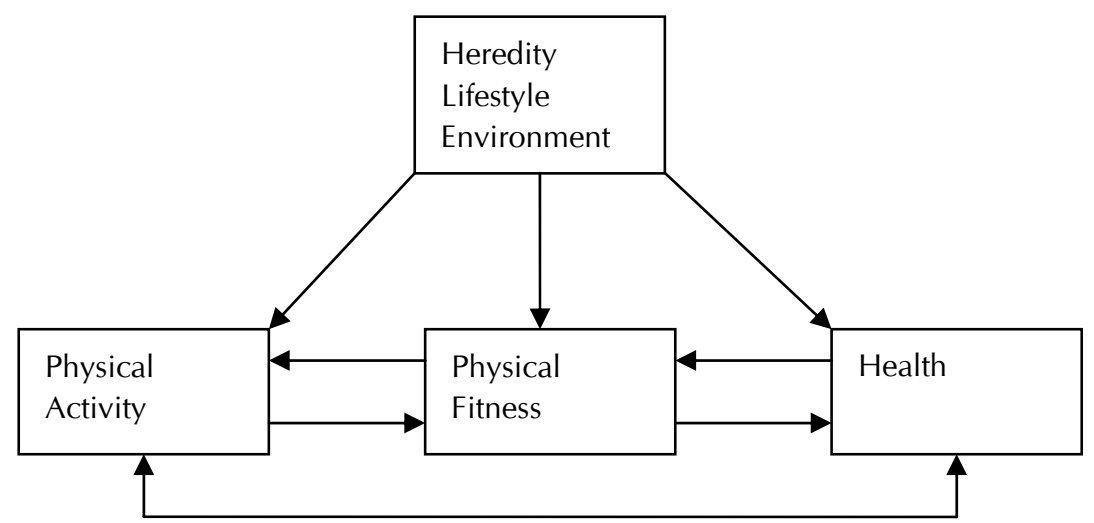

Figure 2 A model describing the complex associations between physical activity, fitness and health in schematic manner (Adapted from Bouchard et al.) (22).

Besides low physical activity levels, also a low cardio respiratory fitness has been linked to a clustering of metabolic dysfunction in young people (27). Since physical activity and fitness are closely connected, it is hard to 
disentangle specific associations. Although in children the causal relationship between physical activity and fitness has long been questioned, it has recently been shown that physical activity has a positive influence on both fitness and the reduction of fatness in both children and adolescents $(86,155)$, also when they are overweight (112). This suggests that physical activity intervention programs are likely to improve the overall health and fitness level. Rizzo et al. (2007) recently showed that fitness itself is more stringently correlated with metabolic risks like insulin resistance and obesity, than with physical activity (155). In Chapter 2, 3 and 4 the relationship between physical fitness and insulin resistance in two different age groups will be investigated.

On a global scale, in children as well as in adolescents and adults the increase in the prevalence of overweight, obesity and insulin resistance and diabetes has clearly been shown $(18,216,217)$. However, the development in the levels of physical fitness and activity are still subject of scientific discussion. The study described in Chapter 6 evaluates the current fitness level of adolescents, and compare this with previous data.

\section{Prevention and intervention}

To date, primary, secondary and tertiary prevention has become an important issue in medicine. This thesis mainly aims to provide data that may facilitate primary and secondary prevention. These types of prevention focus on preventing a disease or try to detect a disease in an early stage and subsequently attempt to reverse the course of a disease. It has previously been stated that the progression from impaired glucose tolerance to beta-cell failure and prevent type 2 diabetes in obese adolescents may be reversed with early treatment (13). To address specific factors in prevention strategies, risk factors for a specific disease have to be known. Additionally, in order to know which groups should be targeted at in prevention activities, groups with an elevated disease risk should be identified. In both chapter 2 as chapter 3 and 4 one of the aims is to identify risk factors for insulin resistance and classify target groups for prevention activities.

In most studies described in this thesis the study population consists of adolescents. Although prevention activities should start as early in life as possible, adolescence should be considered as an important period to study prevention activities. Adolescence is a crucial period in which children turn into adults and start to make their own lifestyle choices (176). With regard to the subject of this thesis, it is furthermore remarkable that in this period of life physical activity decreases (219). The word "adolescence" originates 
from the Latin 'adolescere', which means growing up. The age range belonging to adolescence definition varies between cultures, although the WHO defines adolescence as the period between the 10th and 20th year of age.

Although overweight and insulin resistance prevention activities aiming at this age group are still limited, several intervention programs have been developed, because the increasing prevalence of obesity and diabetes not only concerns the affected individuals, but also impacts society as a whole. At present, the direct costs of overweight in the Netherlands are estimated to be three to five percent of all the resources spent on health care (203). This has stimulated the design of community based intervention programs to counteract the anticipated obesity "explosion". Since much resources are spent on intervention programs, even though many of them are not evidence based, the effect of one specific practice based program (Realfit) aiming to reduce obesity in childhood was studied in Chapter 7. The result of this study gives information about the effectiveness of these types of programs, and may be helpful in improving the current program.

\section{Aim and outline of the thesis}

The decrease in overall physical activity in combination with the increase in the number of adolescents with overweight and obesity, have been suggested to play a dominant role in the development of insulin resistance, the MetS and type 2 DM. Scientific results studying risk factors for insulin resistance are, however, generally based on studies in specifically selected, mostly overweight, populations. For prevention activities, it is important to have more in depth knowledge concerning risk factors in an early, predisease state. Therefore, in the studies described in the current thesis the association between physical fitness, body composition and insulin resistance has been investigated in "healthy" Dutch adolescents.

The main aim of this thesis is to investigate possible associations between body composition (fat mass and fat free mass), aerobic fitness and insulin resistance in Dutch adolescents living in the province of Limburg.

In the different chapters, specific components of the association between body composition, fitness and health have been studied. In the first two studies the prevalence of insulin resistance has been investigated in two different age groups in a subpopulation of a birth cohort. In Chapter 2 insulin resistance and potential predictive parameters of insulin resistance at 
the age of 7 to 8 years have been investigated. In Chapter 3 follow up data are presented which were derived from the same group as described in chapter 2, only now at the age of 13 . In both chapters 2 and 3 specific factors are investigated as predictors for insulin resistance (as estimated by HOMA). The importance of studying these hypotheses in a cohort population is that the development and tracking of insulin resistance can be studied as well. Tracking provides information about the predictability of insulin resistance at the age of 7 for the insulin resistance at the age of 13 and this may give an indication at which age prevention measures are most likely to be effective. Chapter 4 presents the prevalence of an elevated glucose concentration in a non selected group of healthy Dutch adolescents and tries to unravel the associations with several potential risk factors. This prevalence is compared to international studies and should be regarded as baseline data for The Netherlands.

To counteract obesity and insulin resistance, physical fitness has been suggested to be one of the predictive factors (60). Chapter 5 describes the association between body build and aerobic test performance in a non selected group of healthy adolescents. In this study body build/composition has been expressed by the fat mass index and the fat free mass index, to overcome certain limitations of BMI, as described before (210). A decreased fitness level could be one of the factors associated with the upcoming prevalence of insulin resistance. As there is a lack of recent data about physical fitness, the aerobic test performance level of Dutch adolescents is described in Chapter 6 and compared to previous data.

In Chapter 7 the effectiveness of a practice based program aiming to improve overall health in a group of overweight adolescents, has been studied. There are several practice based programs that try to counteract the obesity and insulin resistance epidemic, but it is unknown whether these programs are effective. This was the reason to evaluate one of them (Realfit). Finally, in Chapter 8 the results of the thesis are integrated and discussed in the light of present literature regarding body composition, insulin resistance and fitness in adolescents. 


\section{CHAPTER 2}

Insulin resistance, physical fitness, body composition and leptin concentration in 7-8 year old children

Jantine D. Slinger, Eric van Breda, Hans Keizer, Patrick Rump, Gerard Hornstra, and Harm Kuipers

Journal of Science and Medicine in Sport. 2007 In press 


\section{ABSTRACT}

Objective The aim of this study was to investigate the association between insulin resistance and physical fitness, leptin concentration, body composition and family history for diabetes in non selected young children.

Study design Physical fitness, fasting plasma glucose, insulin and leptin concentrations, anthropometric characteristics and medical history were available in 257 7-year-old Dutch children. Correlations with the homeostasis model assessment (HOMA) index for insulin resistance were studied. A multiple regression model was calculated for HOMA.

Results The differences between children with or without a family history for diabetes were not significant. Boys scored higher on glucose concentration and aerobic fitness and lower on sum of skin folds and leptin concentration $(p<0.05)$. After adjustment for sum of skin folds, HOMA was significantly associated with leptin in both genders (boys $r=.184 p=.031$; girls $r=.430 p=.000$ ). The association between physical fitness and HOMA was mediated by the sum of skin folds. The associations were stronger in girls than in boys. In the regression model $(R 2=.205)$ the leptin concentration was the only significant predictor.

Conclusions The influence of family history for diabetes on insulin resistance is shown as a trend at this age. The findings suggest that plasma leptin concentration is independently associated with the development of insulin resistance in a nonselected pre-pubertal population. The association of physical fitness with insulin resistance seems to be mediated by the sum of skin folds. 


\section{INTRODUCTION}

To date, scientific reports show an alarming increase in the incidence of type 2 diabetes mellitus (type 2 DM) in adolescents and (young) children in the USA $(8,65,119,172)$. Until recently only $1-2 \%$ of young diabetic patients were considered to suffer from type $2 \mathrm{DM}$. Recent epidemiological studies, however, indicate that, depending on ethnicity, up to $45 \%$ of children with newly diagnosed diabetes mellitus suffer from type 2 DM (67). In the USA the prevalence of type $2 \mathrm{DM}$ in children and adolescents has been estimated to range in various populations between 2 and 50 cases per 1000 people, representing a 10-fold increase during the past two decades (187). In the Netherlands, type 2 DM in children has, thus far, rarely been diagnosed. However, the prevalence of obesity, a major risk factor for the development of type $2 \mathrm{DM}$, has increased more than two-fold from 3-5\% in 1980 to $7-12 \%$ in 1997 in 5 to 11 -year-old boys (91). Whether this trend is associated with an increase in insulin resistance and type 2 DM in adolescents in the Netherlands remains to be established. Since the onset of type 2 DM appears to shift to early life, it is important to study the risk factors for the disease in childhood.

Focussing on body composition as an important risk factor for type 2 DM, it has been shown that body fat, or more specifically visceral fat, was associated with insulin sensitivity in early pubertal children (80). Besides obesity, several other risk factors for the development of insulin resistance have been suggested in youth (60). For instance, it has been shown that the family history for insulin resistance is an important risk factor (47). In line with body composition (60), leptin has also been shown to be related to insulin resistance (157), even when adjusted for adiposity (179). Furthermore, aerobic capacity has been suggested to be a risk factor for diabetes (115), however in youth the association of insulin resistance with aerobic capacity has only been studied in groups of overweight children. These studies showed that the aerobic capacity was not independently related to insulin sensitivity $(15,167,168)$. A limitation of the majority of the studies is that they have been performed in children who are post pubertal or have already entered the puberty phase (179).

Only a few studies have been performed in pre-pubertal children in this field of research. $\mathrm{Ku}(2000)$ et al. showed in a group of 68 pre-pubertal children that insulin resistance was associated with physical activity, but not with physical fitness (114). Roemmich (1998) showed in 28 pre-pubertal children that adiposity is associated with the leptin concentration, and that energy expenditure may be negatively related to leptin levels (157). The studies in pre-pubertal children are relatively small, and none of them combined insulin resistance with physical fitness, body composition and 
leptin concentration. Therefore, the major aim in this study was to elucidate the associations between insulin resistance on the one hand and aerobic capacity, body composition and leptin concentration on the other hand in a substantial group of 7 and 8 year old Dutch children who were not preselected for obesity.

\section{MATERIAL AND METHODS}

In total, 257 children (141 boys and 116 girls) from a previously described non-selected birth-cohort $(n=752)$ were included (163). All children were born between December 1990 and January 1994 in the province of Limburg, the Netherlands. The data described in this study were collected between 1997 and 2000. In the present study the focus is on the association of physical fitness, body composition and leptin concentration with homeostasis model assessment insulin resistance measured at the age of 7 . Both the children and their parents were fully informed of the nature and purpose of the present project, and written informed consent was obtained. The experimental protocols were approved by the Medical Ethics Committee of Maastricht University and the Academic Hospital of Maastricht.

At approximately seven years of age, all children visited the outpatient clinic. To estimate fitness, the children performed a physical exercise test on a motor-driven treadmill, which was programmed according to the Bruce protocol $(32,45)$. In the Bruce protocol every three minutes the speed is increased by $1.3 \mathrm{~km} / \mathrm{h}$ and the grade with $2 \%$, starting with $2.8 \mathrm{~km} / \mathrm{h}$ and a grade of $10 \%$ (32). Prior to the test, each child was allowed to practise at various speeds and gradients for approximately five minutes. Subsequently, the test procedure was explained. The children were instructed to walk and run on the treadmill as long as possible. The test was terminated when the child was unable to continue despite strong verbal encouragement. Heart rate was monitored continuously during the test with a telemetric Polar system (Polar Electro OY, Finland) and recorded automatically every 15 seconds. If the heart rate exceeded the age predicted maximal heart rate minus two standard deviations or levelled off prior to the end of the test, or when the subject showed clinical signs of intense effort, the performance was accepted as maximal or at least as near maximal (161). Non maximal data were excluded from the analyses. Endurance time (the time in minutes between start and termination of the exercise test) was used as a functional measure of physical fitness. 
As described in detail before (163), fasting blood samples were collected in EDTA-treated vacuum tubes. Plasma was separated from blood cells by centrifugation. Next, the samples were stored in small aliquots at $-80^{\circ} \mathrm{C}$ until analysis. Glucose was determined enzymatically (Glucose HK-method, Hoffmann-La Roch, Basel Switzerland) and specific insulin was measured as described by Ruige at al (160). Plasma leptin concentration was determined using a human leptin radioimmunoassay (Linco Research, St. Charles, MO, USA). An estimate of relative insulin resistance was calculated from the fasting insulin and glucose concentrations using the homeostatic model assessment equation for insulin resistance (HOMA IR = fasting glucose $(\mathrm{mmol} /$ liter $) \times$ fasting insulin $(\mu \mathrm{U} / \mathrm{ml}) / 22.5)(123)$.

Body weight (BW) was determined to the nearest 100 gram on a digital scale (Seca 770, Hamburg, Germany), bare footed in light underwear. Height was measured to the nearest millimeter using a wall-mounted stadiometer (Holtain LTD, Crymych, UK). Body mass index (BMI) was calculated as body mass $(\mathrm{kg})$ divided by squared height $\left(\mathrm{m}^{2}\right)$. Biceps, triceps, sub scapular and crista skin fold thickness was measured in duplicate to the nearest $10^{\text {th }}$ of a milimeter on the left side of the body, using a skin fold caliper (Holtain Ltd., Crymych, U.K.). Anthropometric measurements were performed by one physician (PR). The family history for diabetes was obtained through a family disease history interview with (one of) the parents.

Statistical analyses were performed using SPSS software (SPSS Inc. Chicago, IL 60606, USA). Group characteristics are expressed as means ( \pm SD). Gender differences were tested with an independent samples t-test. Pearson correlation coefficients were calculated to characterize the associations among the variables of interest. To adjust for other variables partial correlations were calculated. Backward multiple regression analysis was performed to investigate which model could be predictive for the insulin resistance. The variables tested in this analysis were; family history for diabetes, leptin concentration, endurance time, age, gender and anthropometry defined as skin fold thickness, BMI and trunk/extremity ratio in three different analyses. Since the residues of the HOMA insulin resistance variable were not normally distributed the variable was log transformed before executing regression analyses. The collinearity between the predictors was tested by calculating the variance inflating factors (VIF). Additionally, the differences between the children with a positive and negative family history of diabetes will be investigated with the independent samples t-test. The statistical level of significance was set at $p<0.05$. 


\section{RESULTS}

In this study 141 boys and 116 girls with an average age of 7.25 years $( \pm$ 0.26) were included. The average HOMA index was 1.10, which was similar for boys and girls. The boys scored significantly higher $(p<0.05)$ on glucose concentration and endurance time, whereas girls scored significantly higher $(p<0.05)$ on skin fold thickness and leptin concentration. No gender differences were found in weight, BMI, insulin concentration (table 1). Twenty-two of the 257 participants $(8.6 \%)$ were overweight based on the reference values of Cole (2000) (41). Among 83 children $(32 \%)$ there was a first or second degree family member suffering from diabetes (type 1 or 2 ).

Table 1 Population characteristics and results

\begin{tabular}{lllll}
\hline & Total $(\mathrm{N}=257)$ & Boys $(\mathrm{N}=140)$ & Girls $(\mathrm{N}=117)$ & P-value \\
\hline Age (years) & $7.26(0.26)$ & $7.18(0.24)$ & $7.31(0.22)$ & .898 \\
Weight $(\mathrm{kg})$ & $25.17(4.11)$ & $25.40(4.04)$ & $24.90(4.21)$ & .340 \\
BMI $\left(\mathrm{kg} / \mathrm{m}^{2}\right)$ & $15.51(1.71)$ & $15.49(1.75)$ & $15.53(1.67)$ & .858 \\
Sum of skin folds $(\mathrm{mm})$ & $27.20(9.98)$ & $25.02(8.98)$ & $29.81(10.30)$ & .000 \\
Trunk/extremity ratio & $0.76(0.16)$ & $0.75(0.14)$ & $0.77(0.18)$ & .200 \\
Endurance time (min) & $10.35(1.54)$ & $10.80(1.53)$ & $9.81(1.37)$ & .000 \\
Glucose $(\mathrm{mmol} / \mathrm{l})$ & $4.68(0.35)$ & $4.72(.40)$ & $4.63(0.29)$ & .033 \\
Insulin $(\mathrm{pmol} / \mathrm{l})$ & $37.53(16.80)$ & $35.72(15.22)$ & $39.70(18.36)$ & .059 \\
Leptin $(\mu \mathrm{g} / \mathrm{l})$ & $3.70(2.87)$ & $3.04(2.30)$ & $4.49(3.27)$ & .000 \\
HOMA IR & $1.10(0.53)$ & $1.07(0.53)$ & $1.14(0.26)$ & .236 \\
\hline
\end{tabular}

Characteristics and results for the total population $(N=257)$ as well as split for boys $(N=140)$ and girls $(N=117)$. Significance levels are shown for the unpaired t-tests are given for the comparison between boys and girls. BMI = Body Mass Index; HOMA-IR = Homeostatic Model Assessment Index for Insulin Resistance.

No significant differences $(p<0.05)$ in the relevant measurements were observed among the children with or without a family history of diabetes, although the higher values for insulin concentrations and insulin resistance in the group with a positive family history reached near significance $(p=0.067$ and 0.073 , respectively). In the correlation analyses (table 2) it was showns that for boys as well as for girls, HOMA correlated significantly with endurance time, leptin concentration, BMI and sum of skin folds, also after correction for family history of diabetes. Applying correlation analysis with an additional correction for sum of skin folds, only leptin concentration 
remained significantly correlated with HOMA in both genders (boys $r=.184$ $\mathrm{p}=.031$; girls $\mathrm{r}=.430 \mathrm{p}=.000$ ).

Table 2 Correlation coefficients for Insulin Resistance with relevant parameters

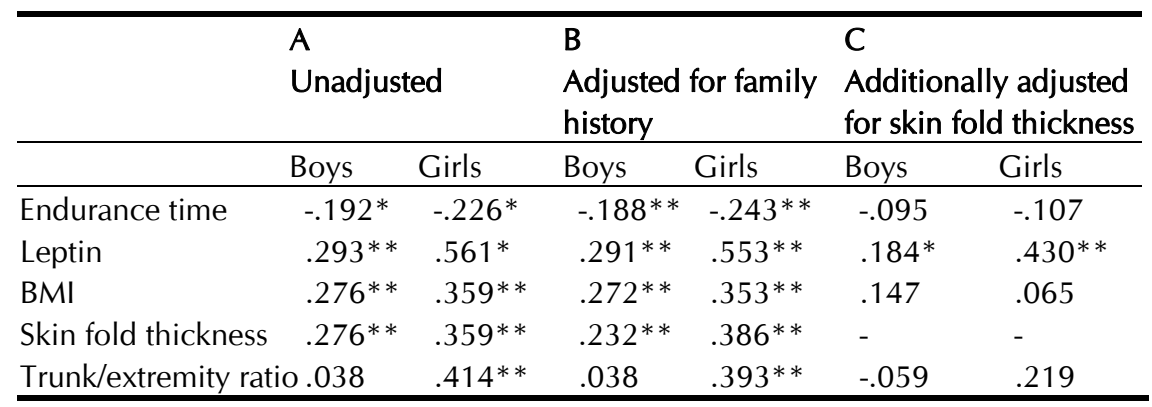

Pearson correlation coefficients for HOMA insulin resistance with endurance time, plasma leptin concentration, BMI, and skin fold thickness. First the uncorrected correlations are shown (A), in the next column the partial correlation is shown with correction for family history of diabetes $(B)$, in the last column the correlations are additionally corrected for skin fold thickness (C). BMI = Body Mass Index; HOMA$I R=$ Homeostatic Model Assessment Index for Insulin Resistance.

The association of HOMA with leptin is more pronounced in girls than in boys. In three different regression analyses (table 3) with family history for diabetes, leptin concentration, endurance time, age, gender and anthropometry consecutively defined as skin fold thickness $\left(R^{2}=.205\right)$, BMI $\left(\mathrm{R}^{2}=.202\right)$ and trunk/extremity ratio $\left(\mathrm{R}^{2}=.202\right)$ as tested predictors, the leptin concentration was shown to be the only significant predictor for the HOMA Insulin Resistance. The VIF showed that the colinearity was acceptable. 
Table 3 Multivariate linear regression analysis HOMA insulin

\begin{tabular}{|c|c|c|c|}
\hline $\begin{array}{l}\text { 3.1Dependent } \\
\text { variable }\end{array}$ & Independent variables & $\mathrm{B} \pm \mathrm{SEE}$ & $\mathrm{p}$ \\
\hline HOMA IR & Gender & $-.050 \pm .064$ & .431 \\
\hline \multirow[t]{4}{*}{$\mathrm{R} 2=.205$} & Family history & $.057 \pm .032$ & .077 \\
\hline & Skin fold thickness & $-.005 \pm .005$ & .336 \\
\hline & Endurance time & $-.020 \pm .023$ & .387 \\
\hline & Leptin concentration & $.090 \pm .018$ & .000 \\
\hline $\begin{array}{l}\text { 3.2Dependent } \\
\text { variable }\end{array}$ & Independent variables & $\mathrm{B} \pm \mathrm{SEE}$ & $\mathrm{p}$ \\
\hline HOMA IR & Gender & $-.047 \pm .066$ & .471 \\
\hline \multirow[t]{4}{*}{$\mathrm{R} 2=.202$} & Family history & $.055 \pm .032$ & .088 \\
\hline & $\mathrm{BMI}$ & $.006 \pm .024$ & .794 \\
\hline & Endurance time & $-.015 \pm .022$ & .496 \\
\hline & Leptin concentration & $.075 \pm .016$ & .000 \\
\hline $\begin{array}{l}\text { 3.3Dependent } \\
\text { variable }\end{array}$ & Independent variables & $\mathrm{B} \pm \mathrm{SEE}$ & $\mathrm{p}$ \\
\hline HOMA IR & Gender & $-.051 \pm .064$ & .427 \\
\hline \multirow[t]{4}{*}{$\mathrm{R} 2=.202$} & Family history & $.054 \pm .032$ & .092 \\
\hline & Trunk/extremity ratio & $.063 \pm .219$ & .773 \\
\hline & Endurance time & $-.016 \pm .022$ & .477 \\
\hline & Leptin concentration & $.075 \pm .013$ & .000 \\
\hline
\end{tabular}

Multivariate linear regression analysis for HOMA insulin resistance with gender, family history, anthropometry (skin fold thickness (3.1), BMI (3.2), trunklextremity ratio (3.3,), physical fitness (as measured by the endurance time) and leptin concentration as possible predictors. BMI = Body Mass Index; HOMA-IR = Homeostatic Model Assessment Index for Insulin Resistance.

\section{DISCUSSION}

The aim of the present study was to investigate the association between insulin resistance as measured by the HOMA index and family history for diabetes mellitus, physical fitness as measured by endurance time, leptin concentration and body composition in a non-selected group of young children. The results suggest that, even after correction for sum of skin folds, leptin is associated with insulin resistance at this young age. This was especially true for the female group. The aerobic capacity (measured by 
endurance time) appears to be indirectly associated with the HOMA for insulin resistance through sum of skin folds.

Although it is often stated that a positive family history for diabetes is an important risk factor for the development of insulin resistance and diabetes, only a trend was observed for differences in insulin concentrations and in HOMA index among the children with either a positive or negative family history of type $2 \mathrm{DM}$. The results of other studies in pre-pubertal children are inconsistent as far as such statement is concerned. For instance, Danadian et al. (1999) have indicated that a positive family history of type 2 DM is an important risk factor for the development of insulin resistance at this age as well (47). However, this was a study in only 23 ten-year-old children. In contrast with these findings, Goran et al. (2003) showed that a positive family history for diabetes does not have a significant association with insulin resistance in young children, which is in line with the current results (82). It is, however, feasible that at an older age family history may appear to have a significant relationship with insulin resistance. For these reasons, the analysis was adjusted for family history in the partial correlation analysis.

The HOMA insulin resistance is significantly correlated with endurance time and this association was independent of the family history for diabetes in both genders. It can be expected that this association was mediated by the anthropometry, because the Bruce protocol used to investigate the aerobic capacity is a weight bearing activity and insulin resistance has been shown to be associated with the anthropometry as well. This mediating role for anthropometry was shown in a regression analysis with adjustment for the sum of skin folds where the association between endurance time and HOMA lost its significance. This is indicative that in this age group the association between physical fitness and insulin resistance is mediated through the sum of skin folds in both genders. These findings are in agreement with the result of a study in a selected group of 11 year old (early pubertal) overweight children (15). In the current study a group of relatively lean subjects was examined and thus the results of the present study show, for the first time, that there is no independent association between physical fitness and insulin resistance in a non-selected population of young children with a relatively lean body composition. It must be realized, however, that physical fitness was defined as endurance time in an incremental exercise test. Since aerobic capacity is also determined by genetic predisposition, endurance time as measured in the present study reflects the amount of daily physical activity only to a certain extent. Since the amount of physical activity was not measured in this study, it would be interesting to study the effect of physical activity in future research. Since it was shown that cardiovascular risk factor profile is less favourable in less active children (6), 
it could be true that the relation between daily physical activity and HOMA index is stronger than with the aerobic fitness as measured by endurance time.

In the correlation analysis the plasma leptin concentration was shown to be the only factor that was significantly associated with HOMA after adjustment for sum of skin folds. Leptin is known as a satiety hormone and is involved in the regulation of appetite and energy balance (19). Since leptin is produced by adipose tissue, leptin levels are usually elevated in obese children and obese adults (179). Furthermore, it has been suggested that hyperleptinemia may be related to insulin resistance in obese individuals, through an increase in the activity of insulin receptor substrate kinase, which inhibits insulin signalling (38). The positive correlation between plasma leptin concentration and insulin resistance as reported in the present study is in line with the findings of Chu et al. (2003) in a group of 13 year old children (36). They stated that leptin may play an important role in the development of hyperinsulinemia and insulin resistance by modulating the activity of insulin receptor substrate in children as well (36, 38). Steinberger et al. (2003) also showed significant associations between leptin and insulin resistance in 13 year old children, even after adjustment for adiposity (179). The obtained correlation in the present study suggests a role for leptin in the development of insulin resistance and diabetes at this young age as well, even adjusted for sum of skin folds. In addition, the importance of the plasma leptin concentration is emphasized by regression analysis for the HOMA index, which showed leptin to be the only significant predictor in the model for insulin resistance. This result was independent of indicators of total body fat and fat distribution. At present no other studies in pre-pubertal non-obese children studying the association between insulin resistance and leptin concentration are known.

As expected, girls score significantly higher on sum of skin folds and leptin concentration and lower on endurance time. The association between leptin and HOMA insulin resistance adjusted for family history and sum of skin folds is stronger in girls than in boys. In a previous study where girls had a higher sum of skin folds and leptin concentration as well, the association between leptin concentration and components of insulin resistance syndrome was not influenced by gender (179). A feasible explanation for the differences in strength of the association between the genders is lacking. The results of this study have some implications for future research and for prevention programs. Although in the investigated age group the association between a positive family history and insulin resistance did not reach the level of statistical significance, the shown trend suggests extra attention should be paid to children with a positive family history already at a young age. Additionally, the prevention focus in this age group should not be on 
physical fitness itself, but probably more on physical activity. It has been shown in pre-pubertal children that physical activity has a positive effect on the body composition (114) and also on leptin concentration (157). In the present study, both these variables were shown to be associated with insulin resistance in pre-pubertal children. The focus of future research in this age group should therefore probably be more on physical activity than on physical fitness.

In conclusion, the results suggest that leptin is associated with insulin resistance already at a young age. The influence of the family history on the insulin resistance is shown as a trend at a young age. Physical activity, and not physical fitness, may play an indirect protective role in the development of insulin resistance in young children by modulating body composition and leptin synthesis.

\section{ACKNOWLEDGMENTS}

The study was financially supported by the Dutch Organization for Scientific Research and by the University Hospital of Maastricht. 


\section{CHAPTER 3}

\section{Development of insulin resistance and related variables between 7 and 13 years of age: a longitudinal study}

Jantine D. Slinger, Eric van Breda, Patrick Rump, Gerard Hornstra, and Harm Kuipers

Submitted for publication 


\section{ABSTRACT}

Aim The main aim of this study was to explore the development of insulin resistance and related variables in a cohort of children between their $7^{\text {th }}$ and $13^{\text {th }}$ year of age.

Methods At 13 years of age, insulin resistance by homeostasis model assessment (HOMA-IR), anthropometry, aerobic test performance (cycle ergometer) and fasting plasma leptin and adiponectin concentrations, were determined in 98 children belonging to a birth-cohort, measured previously at the age of 7 .

Results From 7 to 13 years, insulin and leptin concentrations, insulin resistance and anthropometric parameters increased significantly, whereas the average blood glucose concentration did not change significantly. Adjusted for gender, tracking (calculated by means of linear regression analysis) of anthropometric parameters was moderately high, for HOMA-IR and insulin concentration tracking was moderate, for leptin concentration and aerobic capacity it was low but significant, whereas for glucose concentration tracking was not significant. From multiple regression analysis it appeared that positive family history of diabetes, age and fat mass index were the only significant predictors for insulin resistance at the age of 13 .

Conclusions This longitudinal study demonstrates that, although insulin resistance increased in children between 7 and 13 years of age, glucose concentrations remained stable, possibly due to compensatory elevated insulin concentrations. The insulin resistance at the age of 7 partly explains insulin resistance at the age of 13 . Based on the regression analysis it is suggested that prevention activities at this age should be focused on decreasing fat mass index especially in children with a positive family history. 


\section{INTRODUCTION}

Scientific data show a worldwide, alarming increase in the incidence of type 2 diabetes mellitus (type 2 DM) in (young) children and adolescents (8, 119). In addition, the prevalence of overweight and obesity, major risk factors for the development of type 2 DM, have already reached epidemic levels and are expected to be doubled by 2010 compared with the most recently available surveys from the late 1990s up to 2003 (113). Two recent studies have shown that in school-aged children from urban areas in two different North American countries, obesity was associated with increases in risk factors for the metabolic syndrome $(30,144)$.

Although overweight is one of the prominent risk factors for insulin resistance early in life, several other risk factors have been suggested as well (8). Membership of an ethnic minority, female gender, either small or large for gestational age at birth, a positive family history of diabetes, and elevated leptin concentrations have been identified as risk markers for insulin resistance $(8,173,222)$. Apart from certain risk factors, protective factors have been acknowledged as well. For example, low fat diet and breast feeding have been found to lower the risk of developing insulin resistance $(97,222)$. Recently, also adiponectin has been suggested to be an important protective factor in the development of insulin resistance (169, 178). Furthermore, it has been shown that physical activity and aerobic capacity are important protective factors of insulin resistance and type 2 DM (103, 134). As reviewed by Hussain et al. (2007), the preventive effect of physical activity is mediated by a direct effect on insulin resistance and an indirect effect on weight reduction and better weight management (97). Consequently, the development of insulin resistance and type $2 \mathrm{DM}$ is a complex process in which a combination of genetics (142), risk factors and protective factors determines whether a person will develop type 2 DM or not.

The global increase in the prevalence of insulin resistance and the alarming increase in overweight in different countries makes it essential to study the longitudinal development of insulin resistance (69). To enable the identification of risk factors for insulin resistance early in life, longitudinal studies should start as early as possible, preferably from birth on. At present, there are only two longitudinal studies evaluating the development of insulin resistance from the pre-pubertal age onwards $(16,81)$. These studies showed that insulin sensitivity decreased during puberty and, interestingly, recovered by the end of puberty. Only one of these papers included leptin concentrations and neither of them included the potential influence of aerobic performance capacity on insulin resistance. Besides this, tracking 
(association between ages) of insulin resistance from childhood to adolescence has not been reported so far.

Therefore, the purpose of the present study is to describe the longitudinal development and tracking of anthropometric parameters, insulin resistance, serum glucose, insulin and leptin concentrations and aerobic test performance at the age of 13 with previous data obtained at the age of 7 (173). Furthermore, the predictive value of potential risk and protective factors for insulin resistance at 13 years of age was investigated. Finally, it is investigated whether a correlation between birth weight and insulin resistance is present at the age of 13 . The results of this study may provide guidelines for early prevention activities.

\section{MATERIAL AND METHODS}

\section{Population and study design}

Children belonging to a previously described non-selected birth-cohort $(n=257)$ visited our outpatient clinic for the last time at the age of approximately seven years $(163,173)$. In the present study, 98 of these children (58 boys and 40 girls) consented to participate. There were no significant differences in base-line measurements at 7 years of age between the participants in the present study and the non-participants, which suggest the absence of selection bias. Both the children and their parents were fully informed about the nature and purpose of the present project, and written informed consent was obtained. The experimental protocols were approved by the Central Committee on Research involving Human Subjects.

\section{Biochemical analyses}

The measurements performed at the age of 7 have been described previously $(161,163,173)$. In the present study the focus is on insulin sensitivity at the average age of 13 , as often expressed as insulin resistance by the homeostasis model assessment for insulin resistance (HOMA-IR)(20) and calculated as (123):

Fasting Insulin concentration $(\mu \mathrm{U} / \mathrm{mL}) \times$ Fasting Glucose concentration $(\mathrm{mmol} / \mathrm{L}) / 22.5$

Blood samples were collected after an overnight fast. After clotting, serum was separated from blood cells by centrifugation, and stored in small 
aliquots at $-20^{\circ} \mathrm{C}$ until analysis. Plasma glucose concentrations were determined using the hexokinase method (Glucose HK 125 kit; ABX diagnostics, Montepellier, France). Insulin concentrations were measured using a radioimmunoassay kit (Insulin RIA-100; Pharmacia, Uppsala, Sweden). Serum leptin and adiponectin concentrations were measured with a double-antibody, sandwich-type enzyme-linked immunosorbent assay that used a monoclonal antibody specific for human leptin (DSL, Webster TX, USA) or adiponectin (Mediagnost, Reutlingen, Germany), respectively. The lower limit of detection for leptin is $0.5 \mu \mathrm{g} / \mathrm{L}$ and the upper limit is $50 \mu \mathrm{g} / \mathrm{L}$. The intra essay coefficient of variation ranged from $4.4 \%$ at $4.7 \mathrm{ng} / \mathrm{mL}$ to $1.5 \%$ at $46.3 \mathrm{ng} / \mathrm{mL}$ and the inter essay coefficient of variation ranged from $4.9 \%$ at $4.7 \mathrm{ng} / \mathrm{mL}$ to $4.2 \%$ at $37.9 \mathrm{ng} / \mathrm{mL}$. The limits of detection for adiponectin are $1-100 \mathrm{ng} / \mathrm{mL}$. The intra essay coefficient of variation ranged from $2.35 \%$ at $5.87 \mu \mathrm{g} / \mathrm{mL}$ to $4.66 \%$ at $14.36 \mu \mathrm{g} / \mathrm{mL}$. The inter essay coefficient of variation ranged from $5.88 \%$ at $4.76 \mu \mathrm{g} / \mathrm{mL}$ to $5.9 \%$ at 11.57 $\mu \mathrm{g} / \mathrm{mL}$.

\section{Anthropometry}

Body weight (BW), height, and percentage fat were determined to assess body composition. Body mass index (BMI) was calculated as the ratio of body weight $(\mathrm{kg})$ and squared height $(\mathrm{m})$. Skin fold measurements to determine percentage fat were done by one observer as previously described (75). The percentage fat was estimated from the sum of the four skin folds (214). Fat mass (FM) was calculated as Percentage Fat $\times$ BW. Fat free mass (FFM) was calculated as BW-FM. FM and FFM were normalized to stature by dividing them by squared length $(\mathrm{m})$, to get the fat mass index (FMI) and the fat free mass index (FFMI) (210). Birth weight has been measured as described previously (162).

\section{Aerobic test performance}

Aerobic test performance was measured with an incremental exercise test on a cycle ergometer (Jaeger ER800, Germany), aiming at maximal performance. The incremental test started at 50 watt and resistance was elevated by 25 watt every 2.5 minute. The test was terminated when the child was unable to continue despite strong verbal encouragement. Heart rate was monitored continuously during the test with a telemetric Polar system (Polar Electro OY, Finland). When the heart rate exceeded 185 beats per minute or leveled off and the subject showed clinical signs of intense 
effort, the performance was accepted as peak or at least as near maximal (161). The maximal achieved watt load was considered as outcome measure. The aerobic capacity was expressed as Wmax/kg.

\section{Family history of diabetes}

In a structured interview with the child and one or both parents, information was collected concerning the family history of diabetes. A positive family history of diabetes was considered to be present when a parent or grandparent of the child had diabetes.

\section{Data handling and statistical analysis}

Differences between variables at the age of 7 and 13 were tested with dependent samples t-tests. Additionally, tracking coefficients between the ages of 7 and 13 years were calculated for glucose, insulin and leptin concentrations, HOMA-IR, body composition and aerobic test performance. The tracking coefficient is a measure of association between two different time points for a functional outcome. Tracking was calculated by means of linear regression analysis, with the normalized values at the age of 13 as dependent variable and the normalized values at the age of 7 as independent variables. Gender was included as a covariable in all analyses. For the biochemical parameters, BMI at the age of 7 was included as an additional covariate. In this method, the regression coefficients $(R)$ can be interpreted as tracking coefficients. The tracking coefficients give information about the predictability of parameters at the age of 7 for the parameters at the age of 13 . The following is a suggested guide to the interpretation of these coefficients: $R<0.30$ : low tracking, $0.30<R<0.60$ : moderate tracking, $0.60<R<0.90$ moderately high tracking, $R>0.90$ high tracking (193). Backward multiple linear regression analysis was performed to explore relations between insulin resistance at the age of 13 and family history of diabetes, leptin concentration, adiponectin concentration, aerobic performance, age, gender and anthropometry defined as fat mass index $(\mathrm{FMI})$ and fat free mass index (FFMI). Family history of diabetes (positive $=$ 1 , negative $=0$ ) and gender (female $=1$, male $=0$ ) were included as dummy's. Normality of the HOMA-IR distribution was judged based on a histogram. Statistical analyses were performed using SPSS software. The statistical level of significance was set at $p<0.05$. 


\section{RESULTS}

In this study, 58 boys and 40 girls with an average age of 12.8 years $( \pm 1.1)$ were included. In six boys and three girls the cycle test was judged as sub maximal. The data of these participants were included in all analyses except for the analyses for aerobic test performance. The characteristics of the group at the age of 13 and differences between boys and girls are shown in table 1.

Table 1 Group characteristics

\begin{tabular}{|c|c|c|c|}
\hline Parameter & Boys $(n=58)$ & Girls $(n=40)$ & $\mathrm{P}^{*}$ \\
\hline Age & $12.73(1.12)$ & $12.79(1.10)$ & .816 \\
\hline \multicolumn{4}{|l|}{ Biochemical Characteristics } \\
\hline Glucose (mmol/L) & $4.60(0.34)$ & $4.59(0.41)$ & .958 \\
\hline Insulin (mU/L) & $7.41(3.34)$ & $10.08(4.19)$ & .001 \\
\hline Leptin $(\mu \mathrm{g} / \mathrm{L})$ & $5.43(5.07)$ & $10.59(7.28)$ & .000 \\
\hline Adiponectin (mg/L) & $23.92(10.52)$ & $27.32(13.02)$ & .156 \\
\hline HOMA Insulin resistance & $1.53(0.72)$ & $2.08(0.93)$ & .001 \\
\hline \multicolumn{4}{|l|}{ Anthropometry } \\
\hline Height (m) & $1.60(0.10)$ & $1.58(0.10)$ & .421 \\
\hline Body weight $(\mathrm{kg})$ & $47.61(10.31)$ & $48.32(9.61)$ & .730 \\
\hline BMI $\left(\mathrm{kg} / \mathrm{m}^{2}\right)$ & $18.45(2.46)$ & $19.18(2.69)$ & .165 \\
\hline Skin fold thickness (mm) & $38.82(22.71)$ & $50.55(21.12)$ & .011 \\
\hline Fat percentage $(\%)$ & $18.21(6.40)$ & $26.40(6.04)$ & .000 \\
\hline Fat mass (kg) & $8.92(4.38)$ & $13.06(4.96)$ & .000 \\
\hline Fat free mass $(\mathrm{kg})$ & $38.68(7.77)$ & $35.26(5.96)$ & .021 \\
\hline FMI $\left(\mathrm{kg} / \mathrm{m}^{2}\right)$ & $14.97(1.32)$ & $14.00(1.24)$ & .000 \\
\hline FFMI $\left(\mathrm{kg} / \mathrm{m}^{2}\right)$ & $3.48(1.67)$ & $5.19(1.86)$ & .000 \\
\hline \multicolumn{4}{|l|}{ Aerobic test performance } \\
\hline Aerobic capacity (Wmax/kg) & $3.36(0.55)$ & $2.88(0.35)$ & .000 \\
\hline
\end{tabular}

Mean and standard deviation (between brackets) are shown for the results of boys and girls at the age of 13. *Student t-test for the difference between boys and girls.

The average HOMA-IR was significantly higher for girls $(2.08 \pm 0.93)$ than for boys $(1.53 \pm 0.72)$. Girls also had a significantly higher percentage fat, fat mass, FMI, FFMI and serum insulin and leptin concentrations. On the other hand, boys had a significantly larger fat free mass and higher aerobic capacity. No gender differences were found concerning age, height, BW, $\mathrm{BMI}$, and serum glucose and adiponectin concentrations. 
For 89 children data were available at 7 and 13 years of age. Table 2 illustrates the comparison of these two measuring moments for the glucose, insulin and leptin concentrations and for HOMA-IR and anthropometric parameters.

Table 2 demonstrates that in this longitudinal comparison both boys and girls scored higher on insulin and leptin concentration and on the HOMA-IR at the age of 13 compared to the age of 7 , whereas the glucose concentration was comparable at both measuring moments. All anthropometric variables increased significantly over time. In the same table the tracking coefficients (association between moments) are shown. The tracking of leptin concentration and aerobic capacity adjusted for gender and baseline BMI, although significant, was low. HOMA-IR and insulin concentration tracked moderately, also adjusted for gender and baseline BMI. The anthropometric parameters tracked moderately high.

Backward multiple regression analysis was used to explore possible predictors for insulin resistance at the age of 13. HOMA-IR was log transformed (In) to normalize its distribution. Age, FMI and family history of diabetes were shown to be the only significant predictors for HOMA-IR in this age group in both regression analyses. This model accounted for $26.1 \%$ of the variance in insulin resistance $(p=0.000)$. The Variance Inflation Factor showed that the multicolinearity of the predictors was within acceptable limits. The results of the regression analysis are shown in table 3.

Table 3 Multivariate regression model for HOMA-IR as dependent variable

\begin{tabular}{lllll}
\hline Dependent variable Independent variable & B (S.E.) & t-statistic & P-value \\
\hline HOMA IR & Constant & -1.267 & -2.162 & .033 \\
$\mathrm{R}^{2}=.261$ & Family history of diabetes & $.218(.096)$ & 2.260 & .026 \\
& Age & $.108(.045)$ & 2.418 & .018 \\
& FMI & $.081(.027)$ & 2.943 & .004 \\
\hline
\end{tabular}

Family history of diabetes, age and FMI were tested as predictors for insulin resistance as measured by HOMA. Leptin concentration, adiponectin concentration, aerobic performance, gender and fat free mass index (FFMI) have been tested as independent variables as well, but did not show significance. 


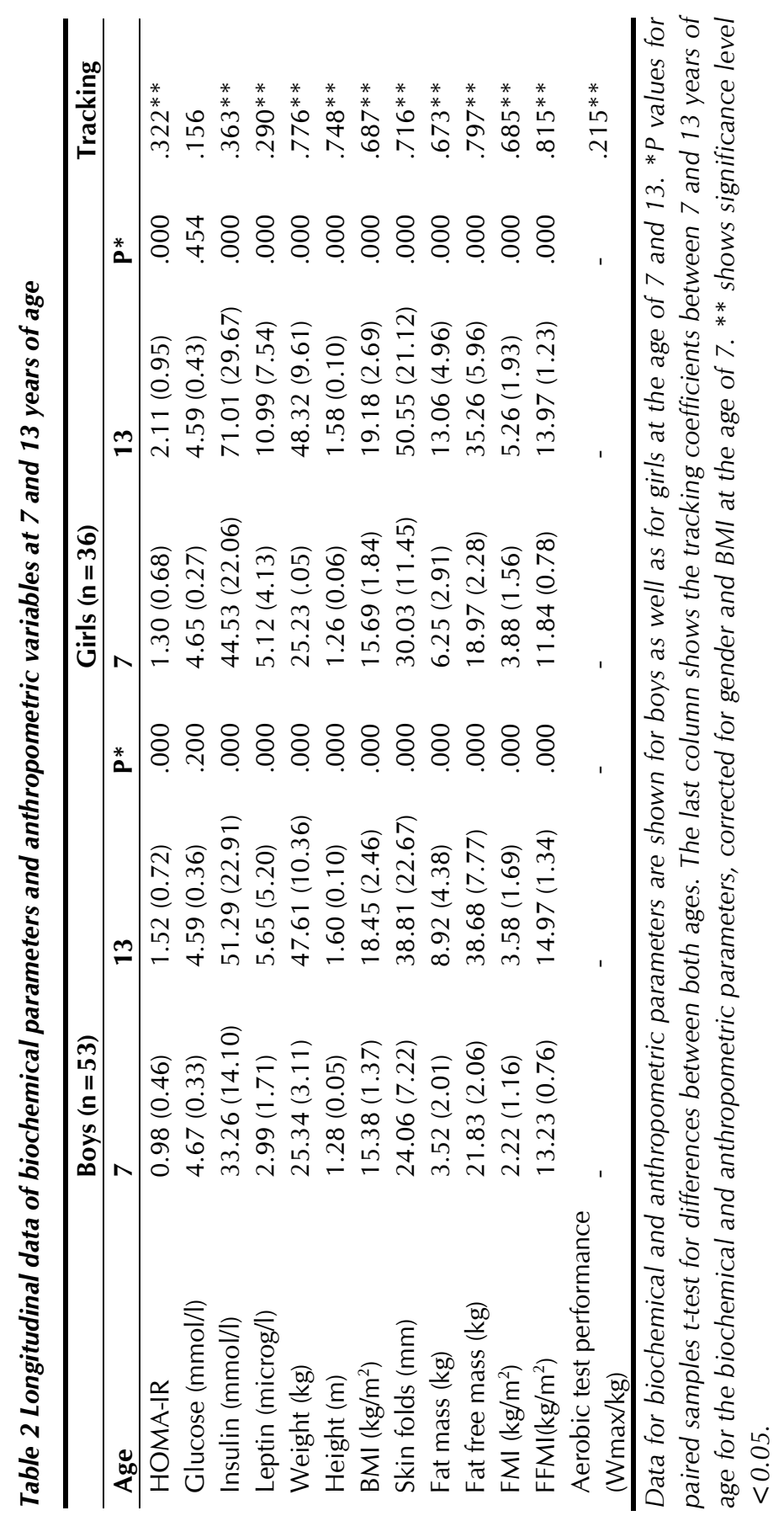




\section{DISCUSSION}

A novel finding of this study is the significant association between ages ('tracking') of HOMA-IR and insulin concentration after adjustment for gender and baseline BMI in Dutch children from 7 to 13 years of age. The tracking of leptin concentration and anthropometric parameters adjusted for gender was shown to be statistically significant. Whereas the glucose concentration remained stable, HOMA-IR, insulin, glucose and leptin concentrations increased over time.

The anthropometric, aerobic and biochemical parameters were within the expected range for this population $(6,28,84,93)$. The average HOMA-IR was 1.52 in boys and 2.11 in girls. These numbers are comparable with a relatively large study by Allard et al. (2003) in 13 year old Canadian children (3). They found a HOMA-IR of 1.66 for boys and a HOMA-IR of 1.90 for girls. The higher HOMA-IR in girls in both studies may be explained by differences in body composition; the current results confirmed that at this age girls have a higher fat mass and a lower fat free mass in comparison with boys.

Taking the previously published data for this cohort (173) into account, it was demonstrated that the HOMA-IR and insulin concentration are rising with increasing age (from 7 to 13 years). Since blood glucose concentration remained stable and insulin resistance increased, it is tempting to speculate that the increased insulin concentration is compensatory and maintains normal blood glucose concentrations. Such a development has also been shown in two earlier longitudinal studies $(16,81)$. Ball et al. (2006) showed in a longitudinal approach that insulin sensitivity decreased during puberty and subsequently recovered by the end of puberty (16). A major drawback of their study was that, due to a high dropout rate, the study population differed considerably between the various stages of the study. The longitudinal study of Goran and Gower (2001) demonstrated in a short-term follow-up period of two years in a group of 30 children, that pubertal transition from Tanner stage I to Tanner stage III was associated with a $32 \%$ reduction in insulin sensitivity (81). They additionally showed increases in plasma glucose and insulin concentrations during pubertal development (81). This is in line with the Dutch population in which insulin resistance as estimated by HOMA-IR was increased by $32 \%$ in boys and by $39 \%$ in girls between the age of 7 and 13. Although, the present study was limited by not measuring pubertal stage by Tanner scores in the 13 year old population, based on their age and average oestradiol $(250 \pm 399 \mathrm{pmol} / \mathrm{l})$ and testosterone levels $(8.10 \pm 8.10 \mathrm{nmol} / \mathrm{l})$ they are expected to fall in the first part of puberty (Tanner stage I, II or III) (154). Thus, the present study proposes that an increase in insulin resistance during the first part of puberty 
is also present in a larger group of European (Dutch) children. Oestradiol and testosterone concentrations were additionally tested as covariates in the tracking statistics, but did not show up as significant covariates for insulin resistance. This suggests that the development of insulin resistance from 7 to 13 years of age was not influenced by pubertal development as measured by these hormones.

Furthermore, it was shown that leptin concentration increased significantly between the age of 7 and 13, with a stronger increase in girls than in boys. Unfortunately, the development over time of adiponectin could not be shown, because this cytokine was not measured at the time the participants were 7 years of age. The increase in leptin concentration may be caused by the increase in fat mass in the population. Until now, causal relations between pubertal development, elevated leptin concentrations and insulin resistance have not yet been established. However, elevated leptin levels have been suggested to be a link between pubertal development and insulin resistance (170). The development of leptin concentrations in this age category has been investigated previously in a cross sectional study (34) and in one smaller $(n=40)$ longitudinal study (1). These studies showed an increase in leptin concentration during puberty in girls, but a stable or even slightly decreased leptin concentration in boys. The latter findings are in contrast with the results of the current study in which the leptin concentrations increased both in both boys and girls. Although in the former studies leptin was not or only weakly associated to the fat mass in boys, in the current study a rather strong positive association between leptin concentration and fat mass in both genders was found $(r=0.66$ and $r=0.78$ for boys and girls, respectively). This association is explained by the fact that leptin is mainly produced in fat tissue (33). Therefore, the normal pubertal increase in fat mass, as found in this study, seems to be a feasible explanation for the increased leptin concentration in boys in the current study.

Adjusted for gender, anthropometric parameters tracked well, whereas insulin concentrations and HOMA-IR tracked moderately, adjusted for gender and BMI. The significant tracking observed for anthropometric parameters confirms earlier studies (205), but to our knowledge tracking of insulin resistance has not been shown before from childhood to adolescence. Although this latter tracking is significant, its strength is only moderate, which suggests that insulin resistance at the age of 7 is only partially predictive for insulin resistance at the age of 13. Additional longitudinal research with larger study populations is needed to shed more light on the predictability of insulin resistance on different ages for the development of type 2 DM. 
At the age of 7 and 13, different aerobic tests were performed. During previous measurements the children were too young to participate in a cycle test measuring aerobic fitness, so an incremental treadmill test was chosen. When the participants were $10+$, they were able to participate in an incremental cycle ergometer exercise test. For this reason the development over time can not be shown, but tracking could be tested. The tracking $(0.215)$ found in the current population was low but significant. This level of tracking is below the tracking coefficient presented by McMurray et al. (2003), which was about 0.500 (126). To the best of our knowledge, until now, this was the only study presenting tracking data for fitness from childhood to adolescence. The lower tracking level may be explained by the different methods used on both moments inthe presentstudy.

In addition to the longitudinal part of this study the cross sectional data were investigated as well and showed that family history of diabetes, age and FMI were significant predictors for insulin resistance in the current 13 year old group (Table 3 ). A positive family history of type 2 DM has clearly been shown to be a risk factor for pediatric diabetes before (8) and therefore, screening should primarily be focused on children with a positive family history. To the best of our knowledge, FMI has not yet been identified as a predictor for insulin resistance and the current finding implies that preventive activities should be aimed at decreasing the FMI in children.

In contrast to the results at the age of 7 , leptin concentration was no significant predictor for insulin resistance at the age of 13 . Furthermore, the adiponectin concentration was not associated with insulin resistance either. So, although these adipokines have been suggested to be associated with insulin resistance (95), the data do not support this hypothesis in the studied age group. In addition, whereas it has been suggested that aerobic capacity is an important protective factor for insulin resistance and type 2 DM (103, 134), in the present study aerobic test performance was no significant predictor for insulin resistance. Finally, birth weight has been suggested to be a predictor for insulin resistance in adolescents (109). In the present study, however, birth weight was not correlated with insulin resistance, neither at the age of 7 , nor at 13 years of age (173). Also after correction for potential confounders such as duration of pregnancy or current body weight, no significant correlation was found. An explanation for the missing associations of insulin resistance with the measured adipokines, aerobic capacity and birth weight $(95,109,134)$, may be the limited degree of insulin resistance in the presently studied group. In this phase of limited insulin resistance the family history for diabetes, age and FMI seem to be more important predictors than the adipokines, aerobic capacity and birth weight. 
In conclusion, in this longitudinal study it was shown that between 7 and 13 years of age the plasma glucose concentration was stable, while HOMA-IR, and insulin, glucose and leptin concentrations increased over this period of time. Furthermore, this study is the first to demonstrate that tracking exists between childhood and adolescence for HOMA-IR and plasma insulin concentrations $(p<0.05)$. Further, tracking was low but significant for plasma leptin concentrations and for aerobic capacity, but not for plasma glucose concentrations $(p>0.05)$. Finally, family history of diabetes, age and FMI appeared to be significant predictors for HOMA-IR at the age of 13 . 


\section{CHAPTER 4}

\section{Elevated glucose concentration and physical characteristics in adolescents}

Jantine D. Slinger, Eric van Breda, Martien van Dongen and Harm Kuipers 


\section{ABSTRACT}

Purpose The aim of this cross-sectional study is to investigate the prevalence of elevated fasting blood glucose concentration in adolescents $(>5.6 \mathrm{mmol} / \mathrm{l})$.

Methods Fasting capillary whole blood glucose concentration was measured in 571 12-15 year old adolescents who volunteered out of a group of 2108 participants in sports tests. In addition, with t-tests and multiple logistic regression analysis body weight and height, aerobic capacity, blood pressure, shuttle run score, sports participation, educational level and food intake were investigated as possible predictors for an elevated glucose concentration.

Results In $15.2 \%$ (S.E. 2.97; C.I. $12.23-18.17 \%$ ) of the adolescents an elevated fasted glucose concentration $(>5.6 \mathrm{mmol} / \mathrm{l})$ was detected in a single measurement. About $3 \%$ showed a confirmed elevated level after repeat measurement. In multivariate logistic regression for elevated glucose concentration an Odds Ratio (OR) of 2.47 (C.I. 1.34 - 4.56) was shown for pre vocational level (lower level) compared with pre university level (higher level), which is a proxy measure of socio economic status. The OR for BMI was 1.08 (C.I. 1.01 - 1.17) and for age the OR was 0.66 (C.I. 0.49 - 0.91). Other parameters and interactions were no significant predictors.

Conclusion In the total population $15 \%$ showed an elevated fasting glucose concentration at the first measurement, whereas in about $3 \%$ an elevated level was confirmed. To prevent type 2 DM in adolescence programs should focus on the adolescents at prevocational educational level and on overweight individuals. Shuttle run score and sports participation did not seem to be associated with glucose concentration. 


\section{INTRODUCTION}

Scientific reports indicate, also in adolescents and (young) children, an alarming increase in the incidence of type 2 diabetes mellitus (type 2 DM) (66). Type $2 \mathrm{DM}$ is a slowly developing disease with a gradual transition from a normal glucose tolerance into the full blown diabetes state. The first stage towards type $2 \mathrm{DM}$ is featured by an increased postprandial glucose concentration, followed by a second stage in which a pronounced elevated fasting glucose level can be found (190). This finally develops into type 2 DM, featured by severe insulin resistance.

To be able to counteract an upcoming pandemic, more information about the current prevalence of the different stages and possible risk factors is needed. The most practical way of screening for (pre)diabetes is by measuring fasting glucose concentrations in large population samples. The American Diabetes Association defined a fasting glucose concentration of $5.6 \mathrm{mmol} / \mathrm{l}$ or above as elevated and a concentration exceeding $7.0 \mathrm{mmol} / \mathrm{l}$ as diabetic (5). In the current study the prevalence of an elevated fasting glucose concentration (second stage) in adolescents is investigated.

In this age group the prevalence of elevated glucose concentration has only been studied in an American population. In the National Health and Nutrition Examination Survey (NHANES) it was shown that in 12 to 19 year old American adolescents $11 \%$ had elevated fasting glucose levels (using $5.6 \mathrm{mmol} / \mathrm{l}$ as cut-off level) (55). Additionally, Allard (2003), showed that in 13 and 16 year old adolescents, boys tended to have higher glucose concentrations than girls (3).

Since prevalence data for an elevated blood glucose concentration among Dutch adolescents are lacking, the first aim of this study was to determine the prevalence of an elevated fasting glucose concentration $(>5.6 \mathrm{mmol} / \mathrm{l}$ (74)) in Dutch adolescents between 12 and 15 years of age. Since body composition is considered as a major risk factor for elevated glucose levels, it is hypothesized that the Dutch prevalence will be below the $11 \%$ found in the NHANES study, because the body composition in Dutch adolescents is supposed to be healthier than the American counterparts (91). Several risk factors for diabetes in children have been identified, such as family history of diabetes, belonging to an ethnic minority, age and female gender (8, 119). Furthermore, physical characteristics like obesity, and blood pressure have also been suggested to be associated with insulin resistance (8). In addition, regular physical exercise and physical fitness do contribute to maintain adequate glucose homeostasis (161). So, besides measuring the prevalence of an elevated glucose concentration, it is additionally investigated whether educational level, BMI, physical fitness, blood pressure, health behavior and family history for diabetes may predict an 
elevated glucose concentration in adolescents as well. Such information may provide possible clues for prevention of type $2 \mathrm{DM}$.

\section{MATERIALS AND METHODS}

\section{Design and study population}

The cross-sectional study described here has been executed in three different secondary schools located in the southern part of the Netherlands. The school teachers were involved in recruiting the students. The study population was a subpopulation of the adolescents who participated in a fitness test organized by the municipality sports department and was conducted in 2005 and 2006. A total number of 2108 adolescents participated in the fitness related measurements. A subpopulation of 571 adolescents volunteered for glucose measurements. Individuals who showed an elevated glucose level during the first measurement, were invited for a confirmation measurement (74). The participants were $12-15$ years-of-age and attended pre-vocational or pre-university secondary education. Students following pre-vocational education are prepared for practical jobs and, on average, have a lower intelligence level compared to students attending pre university education, who are prepared for academic education. The level of education is considered as a proxy measure for socio economic status. The present study was approved by the Central Committee on Research Involving Human Subjects (The Netherlands, The Hague). Prior to participation, the children and their parents or guardians were fully informed about the study and a signed informed consent was obtained both from the participants and their parents or guardians.

\section{Measurements}

Glucose concentration. After an overnight fast between 8.30 and 9.30 a.m., capillary whole blood was taken in which glucose concentration was measured in duplicate with the Life Scan Ultra (LifeScan, Milpitas, California) This device has been found accurate, also in children between 3 and 17 years-of-age $(2,49,70,207)$. Verification of the fasting period was confirmed by asking prior to the measurements by the research assistants. A fasting capillary glucose concentration of $5.6 \mathrm{mmol} / \mathrm{l}$ or above is considered as an impaired fasting glycaemia (IFG), according to the American Diabetes Association criteria (5) was used. A capillary glucose concentration of 7.0 
$\mathrm{mmol} / \mathrm{l}$ or above is an indication for diabetes and a concentration below 3.5 $\mathrm{mmol} / \mathrm{l}$ is considered as hypoglycemic.

Anthropometry. Body mass index (BMI) was calculated as body weight divided by squared height. Height (Seca 206 body meter measuring tape, Hamburg, Germany) and body weight (BW) (Seca 770, Hamburg, Germany) were determined bare footed and in light clothes. Biceps, triceps, sub scapular and crista skin fold thicknesses (Holtain Ltd., Crymych, U.K.) were measured in triplicate. Fat percentage was assessed from the sum of the four skin folds, according to Weststrate and Deurenberg (214). Fat-free mass (FFM) was calculated as '(1-fraction of FM)*BW'.

Aerobic fitness. The adolescents participated in a maximal multistage 20-m shuttle run test (117). Subjects were required to run back and forth on a 20$\mathrm{m}$ course and touch the 20-m line at the same time that a beep was emitted from a tape recorder. The Queen's University of Belfast protocol was used in which the frequency of the sound signals increases in such a way that running speed started at $8.0 \mathrm{~km} \cdot \mathrm{h}^{-1}$ and increased by $0.5 \mathrm{~km} \cdot \mathrm{h}^{-1}$ every minute (191). The outcome of the shuttle run test is the number of completed minutes (with an accuracy of a half minute).

Blood pressure. Blood pressure was measured non-invasively in a seated position on the right arm with the Omron HEM-711AC Blood Pressure Monitor with an appropriate cuff (9). The participants were asked to sit quietly for five minutes prior to the actual measurement.

Health behavior. The children completed a questionnaire regarding physical activity, food intake and (family) history for diabetes. To get an indication of physical activity the participants were asked to indicate how many hours a week they participate in sports (121) as well as the transfer means from home to school. Concerning food intake the daily intake of fruit, vegetable and soft drinks was registered.

Diabetes in family. The adolescents were asked whether they had family members who are diabetic, either type 1 or type 2 .

\section{Statistical analysis}

The frequency distributions of all dependent and independent variables were tested for normality. Differences in physical characteristics and health behavior between students who participated or did not participate in the glucose measurement were tested with independent t-tests (continuous variables) or chi-square test (categorical variables). Values for physical characteristics, health behavior and personal characteristics were compared for participants with an elevated glucose concentration and those with a normal glucose concentration by means of t-tests for independent samples 
(continuous variables) or chi-square tests (categorical variables). The results of these tests were used to select possible predictors $(p<0.10)$ for an elevated glucose concentration. Gender and age were also tested as possible predictors. All predictors were tested in multiple logistic regression analyses with an elevated glucose concentration as dependent variable (Odds Ratio (OR), 95\% Cl). Since BMI and aerobic capacity is gender dependent, interactions for gender and $\mathrm{BMI}$, for gender and shuttle run score were tested. Additionally, the interaction between BMI and shuttle run test result were tested to evaluate the combined influence of fitness and body composition on glucose concentration. The SPSS statistical package, was used and $p<0.05$ was considered statistically significant.

\section{RESULTS}

In table 1 the participants were compared to the non-participants using a ttest. From the total group of adolescents $(n=2108) 571$ individuals participated in the glucose measurement. Those who participated were younger, less tall, had on average a higher educational level, and a lower systolic blood pressure, participated in sports more frequently, consumed less soft drink and diabetes in the family is more prevalent compared to the non participants. In the group of participants $48.3 \%$ were male, 333 followed pre-vocational education and 238 followed pre-university education. The average glucose concentration was $5.3 \mathrm{mmol} / \mathrm{l}$. In $15.2 \%$ $(95 \% \mathrm{Cl} 12.2-18.2)$ of the children a glucose level of $5.6 \mathrm{mmol} / \mathrm{l}$ or above was found. In the normal weight participants this prevalence wat about $13 \%$, whereas in the overweight group this prevalence was about $25 \%$. In none of the subjects a diabetic or hypoglycemic state was found (table 2).

Eventually, $58 \%$ of the subjects with an elevated blood glucose level during the first measurement $(n=56)$ decided to participate in the second measurement and 11 out of these 56 adolescents showed an elevated glucose concentration again. Extrapolating the confirmed prevalence to the present population, it is estimated that about $3 \%$ of the healthy adolescent population has a confirmed elevated fasting glucose concentration. Based on international cut-off values $13 \%$ were overweight and an additional $4 \%$ was obese (41), which is comparable to previous Dutch studies (96). The male adolescents with an elevated glucose concentration had a higher BMI and scored less on the shuttle run test $(p<0.05)$. Adolescent girls with an elevated glucose concentration had lower educational levels and had a lower diastolic blood pressure $(p<0.05)$. There were no differences in other 
measured parameters between adolescents with an elevated or normal glucose concentration.

Table 1 Differences between participants and non participants of the g/ucose measurements

\begin{tabular}{llll}
\hline & $\begin{array}{l}\text { Participants } \\
(\mathrm{N}=571)\end{array}$ & \multicolumn{2}{l}{ Non-participants P-value } \\
& 52 & 44 & \\
\hline Gender (\%girls) & $13.6(1.1)$ & $14.64(1.4)$ & .001 \\
Age (years) & 42 & 17 & .000 \\
Educational level * & $1.62(0.14)$ & $1.64(0.16)$ & .000 \\
Stature (m) & $52.9(13.1)$ & $57.1(12.9)$ & .108 \\
Body weight (kg) & $20.1(3.7)$ & $20.9(3.8)$ & .322 \\
BMI (kg/m $\left.{ }^{2}\right)$ & $117.2(14.2)$ & $122.3(15.3)$ & .001 \\
Systolic Blood pressure $(\mathrm{mmHg})$ & $68.0(9.4)$ & $69.1(9.4)$ & .113 \\
Diastolic Blood pressure $(\mathrm{mmHg})$ & 77 & 64 & .008 \\
Sport participation (\%) & $1.5(0.8)$ & $1.5(0.8)$ & .864 \\
Fruit (pieces) & $4.2(2.6)$ & $4.1(2.7)$ & .969 \\
Vegetables (spoons) & $2.4(2.1)$ & $3.0(2.3)$ & .001 \\
Soft drinks (glasses) & 38 & 33 & .003 \\
Diabetes in family $(\%)$ & & & \\
\hline
\end{tabular}

The table compares the characteristics and fitness parameters of participants in the glucose measurement with adolescents who did not participate in the glucose measurement. Al/ adolescents did participate in the fitness tests. *\%pre-university educational level

Table 2 Prevalence $(95 \%$ Cl) normal and elevated glucose concentrations during the first measurements

\begin{tabular}{ll}
\hline Glucose concentration range & Prevalence $(95 \% \mathrm{Cl})$ \\
\hline $3.5-5.59 \mathrm{mmol} / \mathrm{l}$ & $84.8 \%(81.8-87.8 \%)$ \\
$5.6-7.0 \mathrm{mmol} / \mathrm{l}$ & $15.2 \%(12.2-18.2 \%)$ \\
$>7.0 \mathrm{mmol} / \mathrm{l}$ & $0 \%$ \\
\hline
\end{tabular}

Due to the limited participation rate in the second measurement, further analyses are based on the results of the first measurement. A multiple logistic regression analysis was performed with an elevated glucose concentration (elevated vs. normal during the first measurement) as dependent variable and educational level, BMI, shuttle-run, blood pressure, gender and age as independent variables. When all variables were 
combined in a multiple analysis, educational level, BMI and age were found to be significant contributors to an elevated glucose concentration. These results suggest that the adjusted OR for an elevated glucose concentration is 2.47 for pre vocational level compared with pre university level. An increase of BMI with one unit on the BMI-scale resulted in an $8 \%$ increase in the probability of elevated fasting glucose concentration. Elevations in age with one year within the studied age range decreased the probability of elevated fasting glucose concentration to $66 \%$. Interaction for gender and $\mathrm{BMI}$, gender and shuttle run score, and BMI and shuttle run were not significant, so they were disregarded for analysis. Shuttle run score and blood pressure had no predictive value for elevated glucose concentrations.

Table 3 Multiple logistic regression analysis for glucose concentration (elevated vs. normal)

\begin{tabular}{llll}
\hline & $\mathrm{B}(\mathrm{SE})$ & $\mathrm{OR}$ & $95 \% \mathrm{Cl}$ \\
\hline Educational level & $-.904(.313)$ & 2.470 & $1.34-4.56$ \\
BMI & $.080(.037)$ & 1.083 & $1.01-1.17$ \\
Shuttle run & $-.079(.065)$ & .924 & $0.81-1.05$ \\
Blood pressure & $-.023(.014)$ & .978 & $0.95-1.00$ \\
Gender & $.487(.280)$ & 1.628 & $0.94-2.82$ \\
Age & $-.407(.159)$ & .666 & $0.49-0.91$ \\
\hline
\end{tabular}

Educational level, BMI, shuttle run result, blood pressure, gender and age were tested as predictors for an elevated g/ucose concentration. Significant $O R^{\prime} s(p<0.05)$ are shown in bold. Categorical variables: Educational level $(O=$ pre-university level; $1=$ pre-vocational level); Gender ( $0=$ boys; $1=$ girls)

\section{DISCUSSION}

In this study the prevalence of an elevated fasting glucose concentration (5.6 $\mathrm{mmol} / \mathrm{l}$ or above) was presented for the first time in a group of Dutch adolescents between 12 and 15 years of age. Those with elevated glucose levels may have an increased risk to become type 2 diabetic later in life. The odds for an elevated glucose concentration was significantly associated with $\mathrm{BMI}$, educational level and gender.

In the first measurement $15 \%(\mathrm{Cl}: 12.2-18.2 \mathrm{mmol} / \mathrm{l})$ was found to have elevated glucose levels. Only a limited number (58\%) of individuals with an elevated glucose concentration during the first measurement participated in the second measurement. Therefore the extrapolated prevalence of an elevated glucose concentration of $3 \%$ for the total population should be 
interpreted with caution. Since in other studies only a single measurement was done, the prevalence data found in the first measurement can be compared to other studies.

The WHO recently decreased the cut-off-level for elevated fasting glucose concentration from $6.1 \mathrm{mmol} / \mathrm{l}$ to $5.6 \mathrm{mmol} / \mathrm{l}$ (5). Therefore, the prevalence of $15 \%$ as found in this study was compared to studies which also used the cut-off level of $5.6 \mathrm{mmol} / \mathrm{l}$. To the best of our knowledge the NHANES study is the only study where the new cut-off level has been used. In that study the prevalence among 12 to 19 year old US adolescents was estimated to be $11 \%(55)$, which is slightly lower than the estimation in the current study. A difference between both studies is that in the NHANES study plasma glucose levels have been used, whereas in the current study capillary samples were obtained. The WHO states that the glucose concentration in venous samples is higher than in capillary samples (39). However, the comparability between capillary and venous glucose concentration has hardly been studied in youth. In an unpublished study in adolescents in our laboratory it was found that the mean glucose concentration in capillary whole blood was $0.1 \mathrm{mmol} / \mathrm{l}$ higher compared to the results in venous whole blood. Based on these findings it is concluded that fasting capillary values can reliably be compared to fasting venous glucose values.

Logistic regression analysis showed that a lower educational level, a higher $\mathrm{BMI}$ and a higher age increased the odds for an elevated glucose concentration during a single measurement. Whereas $\mathrm{BMI}$ has been identified as a risk factor before (8), educational level is a new identified risk factor. Educational level is a feature of socio-economic status (24). Although it has been shown previously that socio-economic status is associated with type 2 DM (64) and body composition (73), the influence of educational level has not been studied before. In the current study it has been shown for the first time, that children attending pre-vocational education have a higher odds for elevated glucose concentrations compared to adolescents attending pre university education. This finding suggests that preventive measures should focus more on adolescents attending pre vocational education. When evaluating gender, comparable glucose levels were found in the two gender groups. Therefore it is suggested that diabetes risk is comparable in both genders. In contrast, some studies reported that boys had slightly higher glucose concentrations than girls $(3,55)$, whereas other studies identified female gender as risk factor for type 2 DM (8). Since no differences between both genders were found, it is suggested that interventions to prevent type $2 \mathrm{DM}$ (and associated health risks) should be focused on both boys and girls with a lower educational level and with a high BMI (55). 
Physical fitness has been shown to be associated with insulin resistance (134). In the present study it was found that the shuttle run score was lower in the group of boys with an elevated glucose concentration compared to the group with a normal glucose concentration. However, the shuttle run score, which is a fitness related parameter, was no significant predictor for elevated glucose concentrations. The present results suggest that in the studied population educational level and BMI are stronger predictors for glucose concentrations than fitness. Although it has to be acknowledged that shuttle run results may be converted to estimated VO2 values, in the current study the unconverted shuttle run data were presented in order to stay as close as possible to the original data.

One of the limitations that need to be addressed here it that the glucose measurement may have selection bias because not all children volunteered for glucose measurements. Therefore, health characteristics of the participants were compared with the non participants in the glucose measurement to evaluate possible selection bias. This comparison showed that in general participants were younger, followed a higher level of education, had a lower systolic blood pressure, participated more in sports, drank less soft drinks and had more diabetic family members compared to children who did not participate in the glucose measurement. This suggests that participants in general pay more attention to a healthy life style than non participants. Based on this it is likely that when the total group would have participated, the prevalence of an elevated glucose concentration could have been higher.

Therefore, it is concluded that in a single measurement, $15.2 \%$ of the Dutch adolescents showed elevated glucose concentration levels, whereas about $3 \%$ of the total population showed a confirmed elevated level. These adolescents may be linked to a state of pre-diabetes and are at a higher risk for developing diabetes later in life (5). Data suggest that the glucose concentration is related to age, educational level and BMI, not to shuttle run score and sport participation. This indicates that in the studied age groups attention should be given to the children who are in pre-vocational education (low socio-economic status) and to children who have a higher BMI.

\section{ACKNOWLEDGEMENTS}

We would like to acknowledge Hilde Geraedts and Nadine Costongs for their assistance in collecting the data. 


\title{
CHAPTER 5
}

The effect of body build and BMI on aerobic test performance in school

\author{
children (10-15 years)
}

Jantine D. Slinger, Frans Verstappen, Eric van Breda and Harm Kuipers

Journal of Sports Science and Medicine. 2006 5:699-706 


\section{ABSTRACT}

Objectives Body Mass Index (BMI) has often questionably been used to define body build. The aim of the present study was to investigate the association between aerobic test performance and body build defined as solid, average or slender in 10 to 15 year old children.

Methods Five-hundred-and-two children (53\% boys) aged 10 to 15 years of age were included in the study. Aerobic test performance was estimated with an incremental cycle ergometer protocol and a shuttle run test. BMI and percentage fat (by skin folds) were determined to calculate FMI and FFMI. In the present study body build was defined more specifically using fat free mass index (FFMI $=$ fat free mass normalized to the stature) and fat mass index (FMI = fat mass normalized to stature). The body build of an individual is 'solid' in individuals with a high FFMI for their FMI and is 'slender' in individuals with a low FFMI relative to their FMI.

Results After adjustment for differences in age, gender and body mass the solid group achieved a significantly higher maximal power output $(\mathrm{W})$ and power output normalized for body mass $(\mathrm{W} / \mathrm{kg})$ during the cycle test $(\mathrm{p}<0.05)$ and a higher shuttle-run score $(p<0.05)$ compared to the slender group. The power output normalized for fat free mass (W/kg FFM) was comparable $(p>0.05)$ between different body build groups.

Conclusions This study showed that body build is an important determinant of the aerobic test performance. In contrast, there were no differences in aerobic test performance per kilogramme fat free mass over the body build groups. This suggests that the body build may be determined by genetic predisposition. 


\section{INTRODUCTION}

Data from several recent studies indicate a progressive decrease in physical activity and a parallel decrease in aerobic performance in children and adolescents $(31,100)$. Besides, the body fat content and the prevalence of obesity in children has increased (91). For this reason, the interest in studies aimed at the association between aerobic capacity and body composition in children has increased (59). Westerstahl (2003), for example, who stated that the aerobic fitness in adolescents was decreased in Sweden between 1974 and 1995 (213), suggests that this change was partly due to the increased BMI. Goran (2000) specifies this statement by suggesting that the major influence of body mass on VO2max is explained by FFM. Fatness and excess body mass do not necessarily imply a reduced ability to maximally consume oxygen (78). Hattori (1997) also states that the amount of body fat does not influence the aerobic capacity (90). In contrast, others suggest that body fat itself is associated with VO2max $(62,131,206)$. Overall, the results of these studies are inconsistent.

The methods which are generally accepted to express body composition in both adults and children in these studies are the body-mass-index (BMI) and the percentage fat. The BMI, however, is a crude measure (210). Although $\mathrm{BMI}$ is a general measure of body mass standardised for stature, it fails to account for differences in the two main components of body mass; fat mass and fat free mass. This implies that a high BMI can be caused either by a high fat mass or by a high fat free mass. Besides BMI, the percentage of fat is frequently used as an additional measure for body composition. Hence, percentage of fat itself ignores subject variation in fat free mass, thus individuals may differ in percentage fat either by an identical fat-free mass but different fat mass, or by an identical fat mass but different fat-free mass (210). Thus, both BMI as well as percentage fat may yield a distorted representation of body composition. To overcome this problem, the variables fat free mass index (FFMI) and the fat mass index (FMI) have been introduced $(71,199,211)$. The FFMI is the fat free mass normalized to stature, whereas the FMI is the fat mass normalized to stature (199). In order to combine both measures, Hattori et al. (1997) combined FMI and FFMI in a statistical chart with the FFMI on the x-axis and the FMI on the y-axis (90). In addition, previously the chart has been transformed into a regression model in which the FFMI could be predicted from the FMI (196). Based on the proportion of the FFMI and FMI, the body build of the individuals can be described as 'solid' in individuals with a high FFMI for their FMI and 'slender' in individuals with a low FFMI for their FMI (196). The description of body build based on the combination of FFMI and FMI is a more advanced and integrated way to describe the body build than by only BMI 
and/or percentage fat. Although, there are some more sophisticated measures to investigate body build, like Magnetic Resonance Imaging (MRI) or Dual Energy X-ray Absorptiometry (DEXA), it is not feasible to use these measures in large population based studies.

Since studies on body build are lacking and because some type of selection was introduced in most previous studies, in the present study the impact of body build on aerobic test performance was investigated in a representative cross sectional study population of school children. The aim of this study was to investigate the association between aerobic test performance measured by the maximal power output and the shuttle-run test and body build defined as solid, average or slender in 10 to 15 year old children. The absolute exercise capacity and the capacity normalized for body mass and fat free mass were evaluated.

\section{MATERIALS AND METHODS}

\section{Subjects}

Five-hundred-and-two school children (268 boys and 234 girls) aged between 10 and 15 years from primary and secondary schools in the province of Limburg, the Netherlands were included in the study. For the analyses the group was divided into six subgroups, based on age (10-11, 1213, 14-15) and sex. Data have been collected between 2001 and 2005. Data used for this study were collected within the framework of the physical education classes. Within this framework selection bias is reduced, because all children in a class participate.

All children and their parents were fully informed about the nature and purpose of the study. They had the opportunity to inform the investigator of relevant health problems. Both had, according to the medical ethics committee legal procedures, the right to withdraw from the study at any time.

\section{Anthropometry}

To calculate the BMI, stature and body mass (BM) were determined bare footed in light clothes. The percentage fat was estimated from the average sum of the four skin folds (Holtain Ltd., Crymych, U.K.) because this is valid in a large sample $(200,214)$. Biceps, triceps, sub scapular and iliac crest skin fold thickness were measured in triplicate. Fat-free mass (FFM) was calculated as '( 1 -fraction of FM)*BW'. FM and FFM were normalized to 
stature by dividing them by squared stature, to get the fat mass index (FMI) and the fat free mass index (FFMI) (210). Plotting the FMI on the x-axis and the FFMI on the $y$-axis, subjects with different body compositions could be distinguished $(72,210,211)$. A FFMI-FMl-chart was plotted for the six different age and sex groups, and a regression line with one standard deviation was drawn (figure 1), as has been shown previously by Van Etten et al. (196). Subjects with a deviation of the regression line within one standard deviation were considered to have an average body build. The subjects beyond this prediction interval were assigned to either the slender group (actual FFMI<predicted FFMI) or the solid group (actual FFMI > predicted FFMI) (196). In brief, the subjects in the slender group had a relatively small amount of fat free mass, whereas the solid subjects had a large amount of fat free mass, compared to their fat mass and stature.

\section{Aerobic test performance}

The aerobic test performance was measured with an incremental cycle protocol, in which the participants started with a five minute warm-up at 50 $\mathrm{W}$ for girls and $75 \mathrm{~W}$ for boys (Ergoline, Ergometrics 800, CE 0124). Heart rate was monitored by Polar heart rate monitors (Polar S610). In primary school children the work rate was increased by $25 \mathrm{~W}$ every 2.5 minute. When the heart rate reached 180 beats per minute, the work rate was increased by $10 \mathrm{~W}$ every 2.5 minute, to be able to reach the maximal test performance more precise. In secondary school children work rate was increased by $50 \mathrm{~W}$ every 2.5 minute and by $25 \mathrm{~W}$ when heart rate reached 180 beats per minute. The pedalling rate was $60-80 \mathrm{rpm}$. The result of the cycle test is presented as the maximal achieved work load ('power output').

Additionally, the school children participated in a maximal multistage 20-m shuttle run test (117). Subjects were required to run back and forth on a 20$\mathrm{m}$ course and touch the 20-m line with their foot at the same time a beep was emitted from a tape recorder. The Queen's University of Belfast protocol was used in which the frequency of the sound signals increases in such a way that running speed started at $8.0 \mathrm{~km} \cdot \mathrm{h}^{1}$ and increased by 0.5 $\mathrm{km} \cdot \mathrm{h}^{1}$ every minute (191). The result of the test was expressed in number of stages, which reflected the number of minutes the participants preceded the test.

In both the cycle as the shuttle run test the heart rate measurements were used to evaluate whether the subjects performed maximally. A peak heart rate of $>185$ beats/minute was considered as maximal aerobic performance. Only the data of the subjects who performed maximally were included in the study. During both test verbal encouragements were used to 
stimulate the participants in performing maximally. Both tests were separately completed, with at least one day in between.

\section{Data analysis}

The results are shown as means and standard deviations. To test differences between subjects with an average, solid or slender body build, the total group was divided into six groups based on sex and age categories (10-11, 12-13, 14-15 years of age). Subjects were assigned to one of the defined groups for body build by comparison with their own age and sex group. To test the differences in anthropometric measures between children with a solid, average or slender body build two-way analysis of variance (ANOVA) was used. Post hoc analyses were executed with the Bonferoni test. To test the differences in aerobic test performance analysis of covariance (ANCOVA) was used, with body mass, age and gender as covariates. For statistical analysis SPSS 11.0 was used. A p-value $<0.05$ was considered statistically significant.

\section{RESULTS}

In total 502 school children participated in the study. The cycle ergometer test was performed by 462 subjects whereas the shuttle-run test was performed by 479 subjects. Non-participation in the exercise tests was due to injuries or absence on the day of the test. Stature, body mass and percentage fat were determined in all subjects.

Table 1 shows the anthropometric data for different age groups, for boys and girls separately. An increase in BMI (from 17.3 to $19.5 \mathrm{~kg} / \mathrm{m}^{2}$ in boys and from 17.7 to $20.5 \mathrm{~kg} / \mathrm{m}^{2}$ in girls) and a decrease in percentage fat (from 17.2 to $15.6 \%$ in boys and from 24.9 to $22.9 \%$ in girls) has been shown with an increase in age from 11 till 15 years of age.

Table 2 shows the results of the cycle ergometer test and the shuttle run test for the different age and sex categories.

The FFMI-FMI scatter plots for the six different age/sex groups are shown in figure 1. This figure also presents regression lines for the FFMI predicted from the FMI and the BMI cut-off points for overweight and obesity. Based on the deviation from the regression line the body build of the subjects was classified as solid $(\mathrm{N}=76)$, average $(\mathrm{N}=348)$ or slender $(\mathrm{N}=78)$.

To evaluate the differences between the body build groups, well known measures for body composition are depicted in table 3 for the three groups 

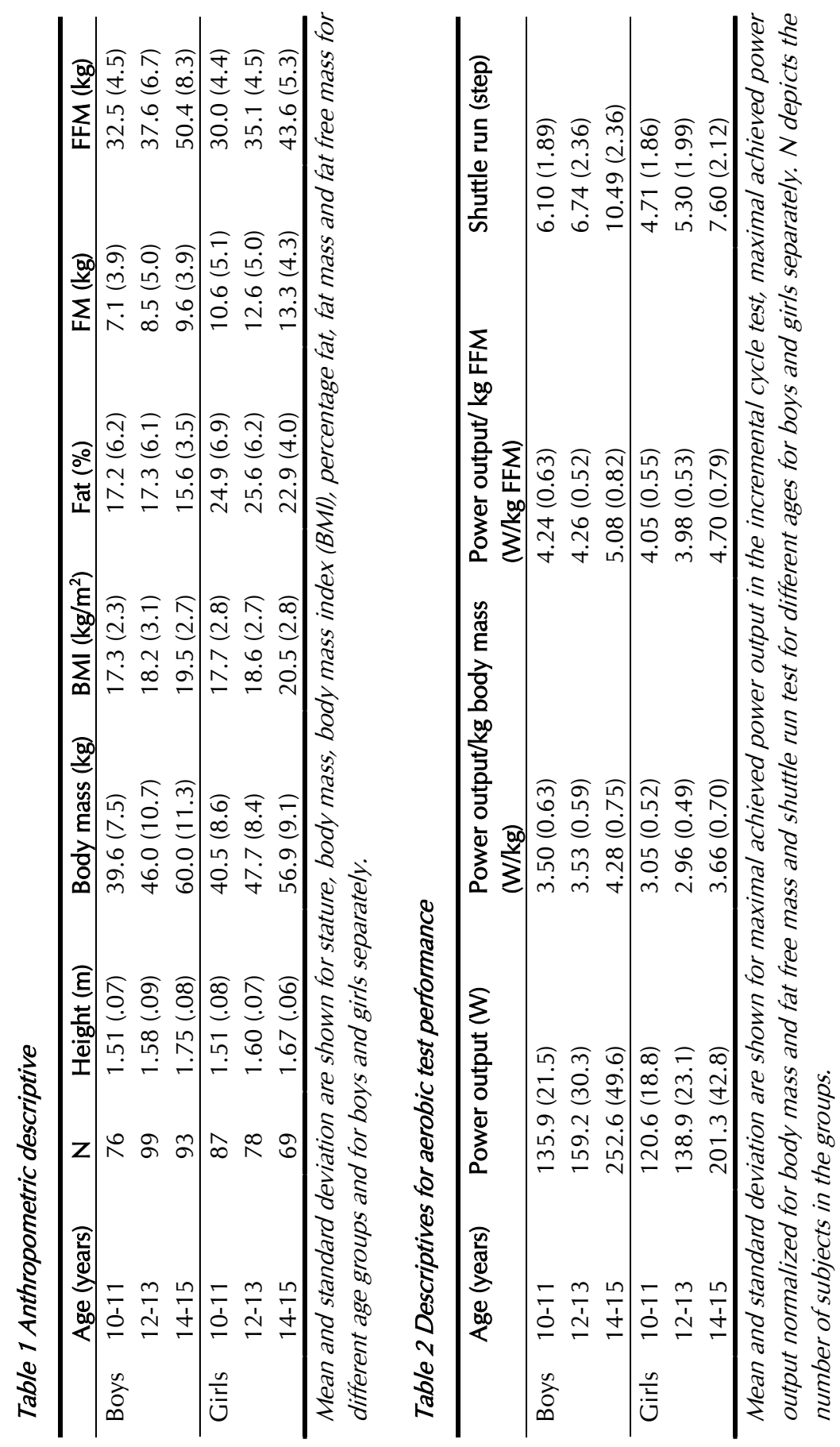
of body build. Table 4 shows the differences in aerobic test performance in the incremental cycle ergometer test and the shuttle run test for the different groups of body build adjusted for age, gender and body mass.

As a result of the methods, the solid group had a higher fat free mass $(p<0.05)$. ANCOVA controlling for age, gender and body mass also revealed group differences $(p<0.05)$ in power output, power output normalized for body mass and shuttle run in favor of the solid group. There were no significant differences between the groups regarding the power output normalized for fat free mass $(p>0.05)$.

Table 3 Differences in anthropometry in body build groups

\begin{tabular}{lllll}
\hline & $\begin{array}{l}\text { Slender } \\
(\mathrm{N}=78)\end{array}$ & $\begin{array}{l}\text { Average } \\
(\mathrm{N}=348)\end{array}$ & $\begin{array}{l}\text { Solid } \\
(\mathrm{N}=76)\end{array}$ & P value \\
\hline Body mass $(\mathrm{kg})$ & $43.8(10.6)^{2,3}$ & $48.2(12.0)^{1,3}$ & $54.5(12.0)^{1,2}$ & .000 \\
\hline Stature $(\mathrm{m})$ & $1.58(0.11)^{3}$ & $1.60(0.12)$ & $1.63(0.11)^{1}$ & .015 \\
\hline BMl $\left(\mathrm{kg} / \mathrm{m}^{2}\right)$ & $17.4(2.7)^{2,3}$ & $18.5(2.8)^{1,3}$ & $20.3(2.9)^{1,2}$ & .000 \\
\hline Fat percentage $(\%)$ & $22.8(7.8)^{2,3}$ & $20.2(6.8)^{1}$ & $18.4(6.1)^{1}$ & .000 \\
\hline Fat mass $(\mathrm{kg})$ & $10.4(5.2)$ & $10.1(5.0)$ & $10.2(4.7)$ & .857 \\
\hline Fat free mass $(\mathrm{kg})$ & $33.4(7.1)^{2,3}$ & $38.1(8.9)^{1,3}$ & $44.3(9.4)^{1,2}$ & .000 \\
\hline
\end{tabular}

Means and standard deviations are shown in measures for body composition for groups based on body build defined by FFMI and FMI. The differences between the values are evaluated with the depicted $P$-value from the ANOVA analysis.

${ }_{1,2,3}$ Represents a significant difference with the value in another column (1=slender, $2=$ average, $3=$ solid) tested by a Bonferroni test.

Table 4 Differences in aerobic test performance in body build groups

\begin{tabular}{lllll}
\hline & $\begin{array}{l}\text { Slender } \\
(\mathrm{N}=78)\end{array}$ & $\begin{array}{l}\text { Average } \\
(\mathrm{N}=348)\end{array}$ & $\begin{array}{l}\text { Solid } \\
(\mathrm{N}=76)\end{array}$ & P value \\
\hline Watt load (Watt) & $156.2(4.0)^{3}$ & $164.9(1.8)^{3}$ & $178.6(4.1)^{1,2}$ & .001 \\
\hline $\begin{array}{l}\text { Power output/ kg } \\
\text { body mass (W/kg) }\end{array}$ & $3.30(.08)^{3}$ & $3.48(.04)^{3}$ & $3.70(.08)^{1,2}$ & .002 \\
\hline $\begin{array}{l}\text { Power output/ kg } \\
\text { FFM (W/kg FFM) }\end{array}$ & $4.37(.08)$ & $4.36(.04)$ & $4.37(.09)$ & .962 \\
\hline Shuttle run (step) & $6.20(.29)^{3}$ & $6.85(.13)$ & $7.50(.29)^{1}$ & .008 \\
\hline
\end{tabular}

Body mass, age and gender adjusted means and standard deviations are shown for aerobic test performance for groups based on body build defined by FFMI and FMI. The differences between the values are evaluated with the depicted $P$-value from the ANCOVA analysis. 1,2,3 Represents a significant difference with the value in another group $(1=$ slender, $2=$ average, $3=$ solid $)$. 

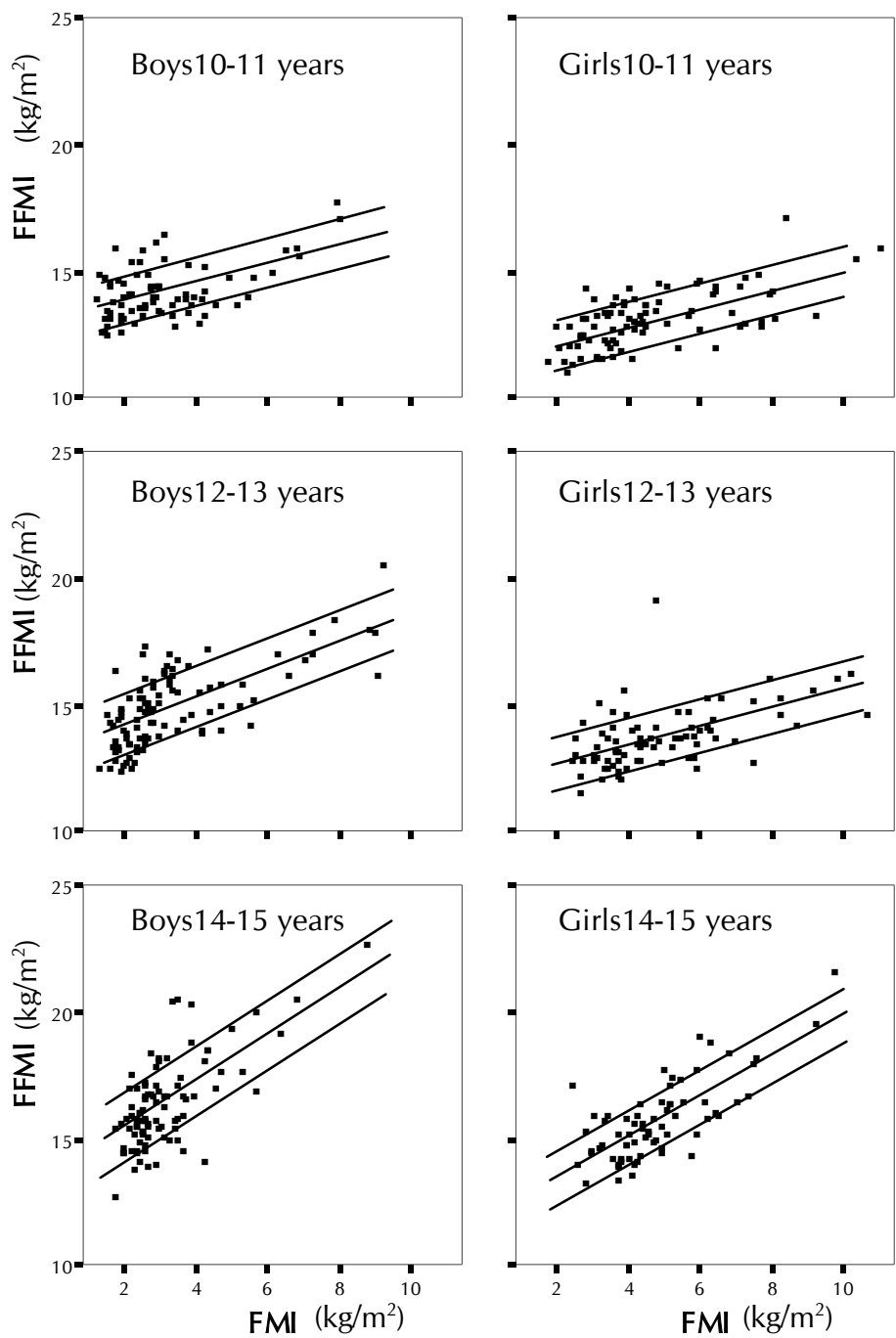

Figure 1 Allocation to body build groups

The figure shows the scatter plots with the FMI on the $x$-axis and the FFMI on the $y$ axis for the specific age and sex groups. The lines represent the regression line for the FFMI predicted from the FMI, with one standard deviation above and beneath. 


\section{DISCUSSION}

The aim of the present study was to investigate the association between aerobic test performance and body build measured by FFMI and FMI in 11 to 15 year old school children. An important finding of this study was that children with a solid body build, thus a high FFMI compared to their FMI, scored significantly higher on the exercise tests, even after controlling for age, gender and body mass. On the contrary, the power output normalized for fat free mass was comparable over the groups.

Since complete school classes were examined in this study selection bias was minimized and a valid representative cross-sectional sample is expected. When the BMI values found in this study were compared to previous found Dutch data in a similar population an increase was found $(75,91)$. This is, however, in line with previous assumptions that the BMI in children is increasing (91).

In this study it was shown that the subjects with a solid body build, who have a higher amount of fat free mass, achieved a higher aerobic test performance as measured by a cycle ergometer test and the shuttle-run test. The results suggest that in the solid group the fat mass appears to be compensated by the amount of fat free mass. Although the total body mass was also higher in the solid group compared to the slender group, the differences in aerobic test performance can not be explained by the differences in body mass because the analysis was corrected for body mass. Therefore, the body build itself seem to be a predictor for the aerobic capacity in adolescents independent of age, gender and body mass.

In addition, the maximal achieved power output normalized for fat free mass was evaluated and there were no significant differences between the groups based on body build. The total fat free mass can be considered as the main functional mass which plays a key role during physical activity (78). The maximal power output normalized for fat free mass is associated with the physical exercise level. This is confirmed by a study of Nikolic et al. (1992) with 15-year-old boys, where it was shown that the maximal aerobic power expressed per kilogramme lean body mass was $20.6 \%$ higher in the trained than in the untrained group (136). From the similar functional capacity of the fat free mass for subjects with a solid, average or slender body build in this study, suggests a similar physical exercise level in the groups. Previously, estimates have been found for the level of sports participation for the different groups of body build (unpublished data). The hypothesis of similar physical exercise level is strengthened by the finding that the sport participation of the solid, average and slender parts of the previous study was comparable between the three groups. This may indicate that the body build is independent of the physical exercise level in 
this age category (11-15 years of age). When the body build is not influenced by physical exercise, it may be determined by genetic factors.

In this study, the generally recognized BMI cut-off points were compared to the FFMI-FMI-charts with regression lines which distinguish between solid, average or slender body build. Children who are overweight based on their $\mathrm{BMI}$ can be classified in the solid, slender or average group. The overweight group consisted of 56 children, in which 13 have a slender, 38 an average and 5 a solid body build. All these children have a high BMI for their age and sex, while the solid children have a relatively high fat free mass compared to their fat mass and stature, whereas the slender children have a relatively low fat free mass (196). It is hypothesized that the slender overweight children are more disadvantaged by their body mass in daily life compared to the solid overweight children who have more fat free mass.

Unfortunately, the group of overweight adolescents was too small to evaluate the impact of body build on aerobic test performance in obesity children. It would be interesting to test this hypothesis in a larger population of overweight children. Besides this, an interesting research question is whether health status in children with a solid body build may even be better than the health status in slender children.

There are some limitations in this study to be acknowledged. Firstly, the used measures have certain limitations. The percentage fat for example could have been measured with a more laboratory based measurement like hydro densitometry weighing, and the aerobic test performance with direct oxygen measurements. However, considering the aim of the study the prime aspect of the study design was to recruit a large random sample of the population. This requires that complete classes of the cooperating schools should be able to participate in the study. Therefore, laboratory measurement of all interesting physiological variables was not feasible. Another limitation is that no information was collected about the maturation status of the participants. Differences in the maturation status may partly explain the differences in exercise performance between the body build groups (7).

In conclusion, in this study it was shown that in the studied age group the aerobic test performance is better in children with a solid body build compared to children with a slender body build. The power output normalized for fat free mass is comparable over the three groups. Since the capacity per fat free mass is associated with exercise training, the results suggest that body build status is determined by genetics, rather than by physical exercise at this age. In future research it is relevant to investigate possible differences in aerobic test performance between overweight children with a solid, average or slender body build, and health differences between different body build groups. 


\section{ACKNOWLEDGEMENTS}

We would like to acknowledge Mirjam Jaarsma, Inge Jansen, Mandy Bruggink, Richard Staats, and Danielle Cluijtmans for collecting the data and the participating school children and teachers for providing the data. 


\section{CHAPTER 6}

Recent performance capacity data for Dutch adolescents

Jantine Slinger, Eric van Breda and Harm Kuipers

Submitted for publication 


\section{ABSTRACT}

Background The aim of this study is to report the current performance capacity in 11 to 15 year old Dutch adolescents.

Methods A total of 509 adolescents participated in an incremental cycle test and 1198 adolescents participated in a shuttle run test. The level of physical effort was assessed by heart rate measurement. In all children height, body weight (BW) and percentage fat (by means of skin folds) were measured.

Results Performance capacity measured on a cycle ergometer increased significantly with age (11-14 years) in boys (140 watt - 280 watt) and girls (123 watt to 171 watt). A similar trend was shown after normalization to body weight $(3.53 \mathrm{~W} / \mathrm{kg}-4.43$ $\mathrm{W} / \mathrm{kg}$ and $3.00 \mathrm{~W} / \mathrm{kg}-3.15 \mathrm{~W} / \mathrm{kg}$, respectively). Shuttle run results increased significantly with age ( $11-15$ years) in boys (7.27 - 9.49 steps) but not in girls (5.72 - 6.17 steps). Differences between boys and girls were significant.

Conclusion Compared to previous data, the absolute performance capacity in both tests remained rather stable during the last 15 years, whereas the performance capacity normalized to $\mathrm{kg}$ body weight was $13 \%$ lower compared to the former data. In spite of a decrease in Watt per kg body weight a weight bearing activity like running was not yet affected by an increased fat mass. Due to differences between countries, timing and methods it is hard to compare studies focussing on performance capacity. For future studies, standardisation in testing and methods should be applied, to be able to validly compare data over time and between countries. 


\section{INTRODUCTION}

Overweight in children and adolescents is a growing problem worldwide (215). It has been reported that the majority of the overweight children will remain overweight in adulthood as well (137). In addition, this secular increase in the prevalence of obesity is associated with major health problems, like type 2 diabetes (type $2 \mathrm{DM}$ ). The rise in prevalence of obesity has been suggested to be at least partly, attributable to a decline in physical activity in adolescents $(181,182)$. To date, it is difficult to accurately describe trends in physical activity in the adolescent population because of the lack of suitable baseline data. Still, there is increasing evidence that physical activity, in particular in clearly defined and more specific contexts such as biking or walking to school, school physical education and organized sport, is diminishing (54). Most of these assumptions are based on Australian and American studies (54). There have been a few studies in European children. A study in Norwegian adolescents showed that there seems to be a downward trend in the level of physical activity from 1950 to 1997 (61). In addition, a decline in the time allocated to physical education and active transportation to school has been reported in the UK (54).

Based on the increase in obesity and a decrease in physical activity, a decline in aerobic fitness of adolescents can be anticipated (58). An appropriate method to determine the level of aerobic fitness is an incremental exercise test, in which the highest achieved exercise intensity is a parameter of functional aerobic capacity. The gold standard to determine aerobic capacity is direct measurement of $\mathrm{VO} 2$ max during an incremental exercise test. A previous study indicated that between the mid- $20^{\text {th }}$ century and 2000, peak VO2 measured with a cycle ergometer test have remained fairly stable in US boys and girls, either presented as absolute $(\mathrm{ml} / \mathrm{min})$ or normalized to body weight $(\mathrm{ml} / \mathrm{min} / \mathrm{kg})$ (58). For large groups direct measurement of VO2max is most of the times not feasible. In larger studies a Wmax cycle tests or a shuttle run tests are applied. In contrast to the finding in US adolescents, Tomkinson et al. (2003) showed that the secular test performance of children and adolescents, measured by a shuttle run test, is declining parallel with an increase in fat mass and a decrease in physical activity (191). Thus, previous international studies show conflicting results, albeit using different methods.

In the Dutch population, an increase in overweight or obesity has also been shown both in children and adolescents (91). Unfortunately, there are no specific recent data for trends in physical activity levels in Dutch adolescents. When extrapolating the data from other countries, it is reasonable to expect a decrease in physical activity in Dutch children and 
adolescents as well. The most recent data regarding exercise tests in Dutch children originate from 1987 and 1985, for shuttle run test and maximal cycle ergometer test, respectively $(84,198)$. Since recent data about performance capacity for the Dutch population are lacking, the primary aim was to present recent results of maximal incremental cycle ergometer tests and shuttle run test as well as body composition data in healthy 11 to 15 year old Dutch adolescents are presented. A secondary aim is to compare the recent Dutch data with data collected in a similar population 15 years before and with comparable populations in other countries.

\section{MATERIALS AND METHODS}

\section{Subjects}

The participants of this study were recruited through primary and secondary schools in the province of Limburg, the Netherlands between 2002 and 2005. All schools located in the city of Maastricht (120.000 inhabitants) were invited to participate in the study; the schools that reacted within the available time frame were included. Two different performance capacity tests were executed. To decrease the burden on participating schools, the children were invited to participate in one of the tests. In order to minimize selection bias, complete school classes were invited for this study. All children and their parents were fully informed about the nature and purpose of the study. They were asked to inform the investigator of possible relevant health problems. Both the parents and the children had, according to the medical ethics committee legal procedures, the right to withdraw from the study at any time. Since the exercise tests were included in physical education lessons none of the children refused to participate or withdrew from the study. The group who participated in the cycle ergo meter test consisted of five-hundred-nine children (274 boys and 235 girls) aged 11 to 14 year of age. The group that participated in shuttle run test consisted of 1176 children (684 boys and 492 girls), aged 11 to 15 .

\section{Anthropometry}

To calculate body mass index (BMI), body height and body weight (BW) were determined bare footed in light clothing. BW was determined on a digital scale (Seca 770 Alpha, Hamburg, Germany). Biceps, triceps, subscapular and crista iliaca skin fold thickness was measured in triplicate (Holtain Ltd, Crymych, U.K.). Fat mass (FM) was calculated from the sum of 
the four skinfolds, according to Weststrate and Deurenberg (214). Fat-free mass (FFM) was calculated as '(1-fraction of FM) $\times$ BW'.

\section{Performance capacity}

Two different maximal exercise protocols were performed for the two groups. During the exercise tests the children wore a Polar heart rate monitor (Polar S610, Finland) to register maximal heart rate during each test. A peak heart rate of 185 or below was considered as sub-maximal and was excluded from analysis.

Cycle protocol. The cycle protocols used were adapted from Arts and Kuipers (1994) (10). The cycle test consisted of a five minute warm-up at 50 watt for girls and 75 watt for boys. In children of primary schools the work rate was increased by 25 watt every 2.5 minute. When heart rate reached 180 beats per minute, work rate was further increased with 10 watt every 2.5 minute. In children from secondary schools the warm-up was similar as described for primary school children. Next, work rate was increased by 50 watt every 2.5 minute and with 25 watt when heart rate had reached 180 beats per minute. Wmax, Wmax/kg and Wmax/FFM were used as outcome parameters. Wmax/FFM was considered to reflect the quality of the fat free mass.

Shuttle run test. A maximal multistage 20-m shuttle run test was used (197). The Queen's University of Belfast protocol was used as described before (191). The highest stage reached in the shuttle run test was used as outcome parameter.

\section{Data analysis}

The results are presented as mean \pm standard deviation for different ages in boys as well as in girls. Differences between boys and girls were tested with an independent t-test. Statistical analysis was performed with the SPSS 13.0 statistical package. A p-value $p<0.05$ was considered statistically significant. 


\section{RESULTS}

The cycle ergometer test was performed by 509 children ( 274 boys and 235 girls). In table 1 (next page) anthropometric data and the results of the cycle test are shown for the different sex and age groups. Twenty six out of 509 children $(4.7 \%)$ reached a sub-maximal heart rate $(<185 \mathrm{bpm})$ on the cycle ergometer test and were excluded from analysis. Eventually, 483 children (261 boys and 222 girls) were included.

Average maximal power output (Wmax) in the study population increased in boys from 140 watt at 11 years of age up to 280 watt at 14 years of age. The Wmax normalized for BW increased from $3.53 \mathrm{Watt} / \mathrm{kg}$ to $4.43 \mathrm{watt} / \mathrm{kg}$ in this age group. There was also an increase in Wmax normalized for FFM (4.28 watt/FFM vs. 5.21 watt/FFM, respectively). In girls the Wmax, Wmax/kg and Wmax/FFM were all significantly $(p<0.05)$ below the values for boys.

The shuttle run test was done by 1176 children between 11 and 15 years of age (table 2 ). Based on the peak heart rate $13.9 \%$ performed sub maximally (heart rate $<185 \mathrm{bpm}$ ) and their results were excluded from analysis. The shuttle run scores are increasing with age in boys, however not in girls. Boys scored significantly higher $(p<0.05)$ compared to girls in all age categories.

Table 2 Results for shuttle run test

\begin{tabular}{llllllll}
\hline & & $\mathrm{N}$ & $\mathrm{BW}(\mathrm{kg})$ & $\mathrm{BMI}\left(\mathrm{kg} / \mathrm{m}^{2}\right)$ & $\%$ fat $(\%)$ & FFM $(\mathrm{kg})$ & SR (steps) \\
\hline Boys & 11 & 27 & $40.6(6.4)$ & $17.3(1.7)$ & $18.0(4.5)$ & $33.2(5.0)$ & $7.27(1.6)$ \\
& 12 & 307 & $49.4(10.0)$ & $18.8(2.5)$ & $17.6(4.9)$ & $40.5(7.1)$ & $8.01(6.3)$ \\
& 13 & 118 & $60.4(13.8)$ & $20.6(3.9)$ & $18.1(5.4)$ & $48.9(8.9)$ & $8.66(2.5)$ \\
& 14 & 86 & $63.7(13.1)$ & $21.2(3.4)$ & $17.2(5.0)$ & $54.3(8.5)$ & $9.55(2.0)$ \\
& 15 & 70 & $67.7(13.7)$ & $22.3(3.9)$ & $17.5(4.9)$ & $55.4(8.4)$ & $9.49(2.4)$ \\
\hline Girls & 11 & 27 & $42.9(8.6)$ & $18.5(2.7)$ & $27.9(6.7)$ & $30.5(4.3)$ & $5.72(1.9)$ \\
& 12 & 227 & $49.8(9.7)$ & $19.4(3.3)$ & $24.5(5.8)$ & $37.3(5.8)$ & $6.25(1.6)$ \\
& 13 & 62 & $55.8(9.7)$ & $20.9(3.3)$ & $25.2(5.3)$ & $41.3(5.2)$ & $6.69(1.8)$ \\
& 14 & 42 & $58.8(6.4)$ & $21.9(2.7)$ & $26.2(4.2)$ & $43.3(4.1)$ & $6.02(1.3)$ \\
& 15 & 48 & $60.9(9.8)$ & $22.8(4.1)$ & $26.6(4.5)$ & $44.5(5.6)$ & $6.17(2.3)$ \\
\hline
\end{tabular}

The table shows the mean results $( \pm S D)$ of $B W, B M I$, fat percentage, FFM and the shuttle run test for boys and girls separately, for the adolescents who participated in the shuttle run test. $N$ is the number of subjects. 


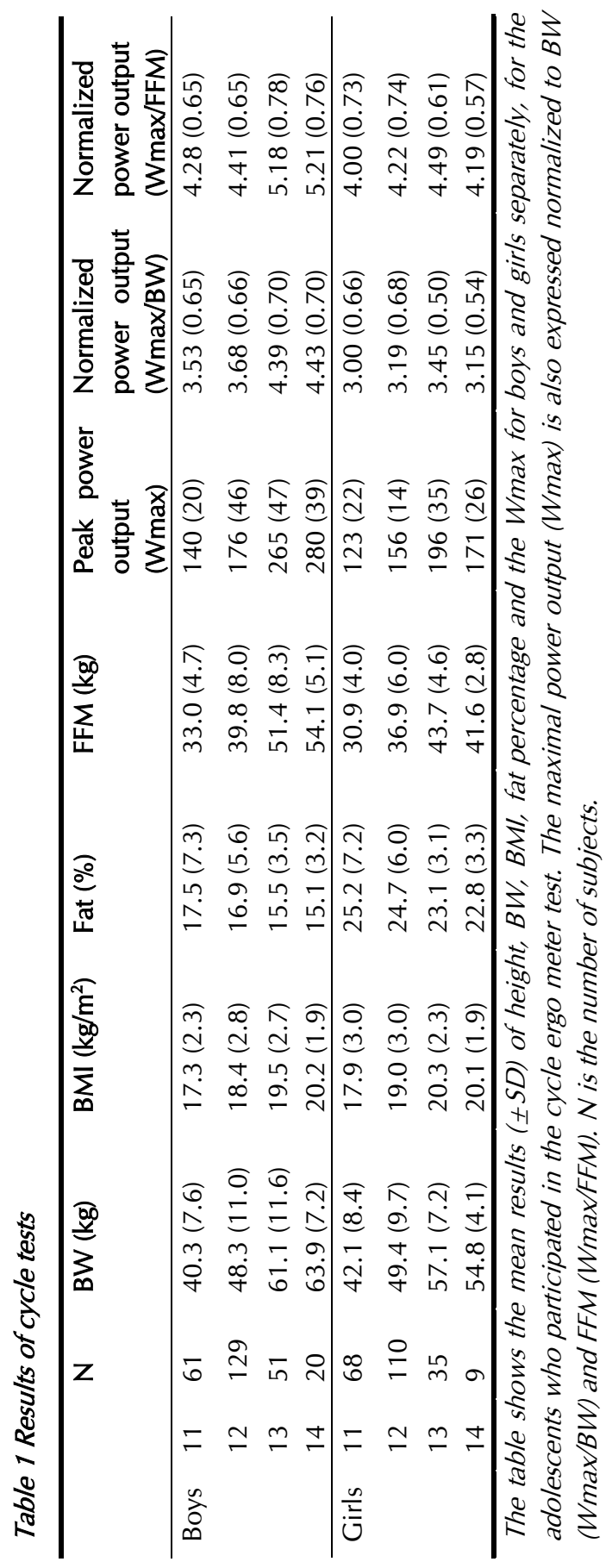




\section{DISCUSSION}

In the present study the latest data on performance capacity in 11 to 15 year old Dutch adolescent boys and girls are presented. Boys had, as expected a higher performance outcome in both the cycle test and the shuttle run test. Furthermore, in both genders an increase in body weight and a parallel increase in peak power output in a cycle test and the score on a shuttle run test were found to increase with age, although in girls the changes in shuttle run test with age was not significant.

The last performance capacity data using a cycle ergometer in Dutch children were collected in 1985 (84). Since both study populations were comparable, as far as age category is concerned, data of both studies in Dutch adolescents can be compared. Due to differences in methods of the present studies and previously published data, a statistical comparison was not possible. In the current study, the peak power output increased from $140 \pm 20$ watt in 11 year old boys to $280 \pm 39$ watt in the 14 year age group. In the girls the mean peak power output at the age of 11 was $123 \pm 23$ watt and increased to $171 \pm 26$ watt in the 14 years old age group. These values of peak power output are in line with the data as published by Gulmans et al. (1997) [10]. However, when expressing the peak power output normalized for body weight $(\mathrm{W} / \mathrm{kg}$ ) a $13 \%$ decrease was found over the 20 year period. A decrease in watt per kg body weight may impact weight bearing activities in daily life, like walking and running. In the present study, functional performance capacity was also measured by employing a shuttle run test. The results of the present study show that in 11 year old boys the maximal stage was $7.27 \pm 1.6$, leveling off around 9.5 stages at the age of 14 , which was a significant increase. In the girls at the age of 11 a maximal stage of $5.72 \pm 1.9$ was reached, stabilizing between 6.02 and 6.69 from the age of 12 . When comparing the present results with the data published by van Mechelen et al. (1987) (198), similar values were found. For a more valid comparison a small adaptation was applied because both tests started with a different running speed ( 8.0 and $8.5 \mathrm{~km} / \mathrm{h}$, respectively). Based on these adapted results, it can be concluded that, in both a cycle test and a shuttle run test, no changes were found over the investigated time spam of 20 years. This indicates that cardio-respiratory fitness in Dutch children did not change significantly over the last 20 years, which suggests that the physical demands on the adolescents in daily life were fairly constant during the last two decades. Simultaneously, body weight and fat mass did increase significantly and the prevalence of overweight and obesity has increased as well (96). Apparently, at present this does not yet affect absolute performance capacity in adolescents. It is, however, likely that a further increase in fat mass will impact physical performance capacity, 
which in turn may decrease the physical activity level, thereby initiating a downward spiral of lower activity and lower cardio-respiratory fitness. When the obesity pandemic proceeds, the effects on weight bearing activities may become more pronounced, which most probably will result in lower shuttle run results. Therefore the development of body composition, fitness and daily physical activity have to be closely monitored.

In addition to the national comparison, the Dutch results can be compared to the international performance capacity data. Although no recent cycle test data (Wmax) could be found, Olds et al. (2006) recently presented worldwide averages per age stratum for shuttle run results (138). The shuttle run scores achieved in the present study were about $9 \%$ higher compared to these worldwide averages. This suggests that Dutch children have a higher cardio-respiratory fitness compared to their counterparts from other countries. A drawback that hinders appropriate comparison is that the data used by Olds et al. (2006) were collected over a large time span from 1981 to 2003. It is hard to study the separate effect of country or changes over time, because the effect of these factors can hardly be separated. The problems in the comparison between different countries can only be solved when performance capacity tests will be more standardized in the future. To be able to validly monitor international trends in aerobic fitness performance capacity tests should be regularly repeated with standardized protocols within the different countries. Tomkinson (2003), however, tried to evaluate time trends in shuttle run score by means of pooled time comparison data of 11 countries over the period 1980-2000 (191). This study suggests a consistent decline in shuttle run score (191). This downward trend in shuttle run results cannot be confirmed by the data of the current study and with those collected in 1987 (198). A possible explanation for these conflicting data is that in the study of Tomkinson the level of physical activity in other countries decreased over time, while it is possible that the Dutch children maintained their physical activity level. Finally, it is valuable in future studies to include the measurement of pubertal development, because body composition is associated with the age of onset of puberty. Nevertheless, Mul et al. demonstrated that the age of pubertal onset in Dutch children is stable since 1980 (132). This suggests that differences over time in performance capacity are not likely explained by different pubertal development. International differences may, however, be disturbed by pubertal differences.

In conclusion, physical performance capacity as measured with an incremental cycle ergometer test and a shuttle run test did increase with age (except the shuttle run result in girls). When comparing the present data with data in Dutch children from 1985 and 1987, no changes in cardio- 
respiratory fitness were found, although percentage fat increased. Probably the increase in fat content did not impact physical activity level yet. This emphasizes the importance to monitor body composition, daily physical activity and fitness level in youngsters.

\section{ACKNOWLEDGEMENTS}

We would like to acknowledge Nadine Costongs, Mirjam Jaarsma, Inge Jansen, Mandy Bruggink, Richard Staats, and Danielle Cluijtmans for collecting the data and the participating schools and students for providing the data. 


\section{CHAPTER 7}

Effects of a 12 week practice based lifestyle program to counteract overweight in adolescents: an intervention study

Jantine D. Slinger, Eric van Breda, Jenny Brouns and Harm Kuipers Submitted for publication 


\section{ABSTRACT}

Background The prevalence of overweight in adolescents is rising worldwide and is one of the major contributing factors for insulin resistance and/or the metabolic syndrome (MetS). The results of a practice-based intervention program (Realfit) on anthropometric, metabolic and aerobic parameters are reported here.

Methods Thirty-two overweight $\left(\mathrm{BMI}=29.2 \pm 4.1 \mathrm{~kg} / \mathrm{m}^{2}\right)$ adolescents (age 12-18) were included in the study. Before, immediately after the 12 week intervention period and 20 weeks after completion of the intervention, anthropometric (BMI, body weight, hip- and waist circumference), metabolic (HOMA insulin resistance, blood pressure, triglycerides, HDL-cholesterol, glucose), and aerobic parameters (VO2max, time spent on physical activity, inactivity and sports), were assessed. General linear model statistics for repeated measures was used to test the development over time.

Results Immediately after the intervention mean body weight, BMI, hip circumference and blood pressure decreased $(p<0.05)$. The prevalence of the MetS decreased from 10 to 7 participants. Increases in $\mathrm{VO} 2 \mathrm{max}(\mathrm{P}=0.055)$ and decreases in physical inactivity $(\mathrm{P}=0.077)$ were consistent but did not reach the significance level. Twenty weeks after completion of the program most outcome parameters had reversed, however, the number of adolescents meeting the criteria of the MetS were further decreased $(n=5)$. Those participants with a lower baseline body weight and $\mathrm{BMI}$ were more responsive to the program and only these subjects were able to improve the level of insulin resistance.

Conclusions The practice-based program improved some of the outcome parameters on the short-term and decreased the prevalence of the MetS. Most results were reversed on the long-term, emphasizing the importance of long-term follow-up programs. 


\section{INTRODUCTION}

The prevalence of overweight and obesity in children and adolescents is increasing worldwide (113). Also, in the Netherlands the prevalence of overweight in early life is increasing rapidly; from 1997 to 2002-2004 the prevalence of overweight has doubled in both genders in most age categories, whereas in the same time frame the prevalence of obesity has increased even more (up to $800 \%$ in certain age categories) $(91,96)$. These numbers reflect the developing overweight pandemic in children and represent a complex and increasing problem which continues into adulthood $(50,137)$. Furthermore, it has been shown that overweight leads to an augmented health risk such as insulin resistance, decreased aerobic capacity, and elevated blood pressure (56). Moreover, overweight is the most important characteristic of the metabolic syndrome (MetS), which is associated with cardiovascular diseases and diabetes mellitus in later life $(99,208,221)$. Depending on the definition used, the prevalence of MetS varies between $5 \%$ in non-overweight adolescents to $30-60 \%$ in overweight adolescents $(42,44,77,101,208)$. Recently it has been shown that the prevalence of the MetS among children and adolescents is increasing worldwide at a rapid rate (94). Unfortunately, in children and adolescents consensus about a definition of the MetS is lacking, probably because growth has an effect on some of the markers (Fernandez, 2004). Recently, Jolliffe and Janssen (2007) published age-specific (12-20 years of age) adolescent MetS criteria that are linked to adult MetS (101). In the current study the definition and cut-values as proposed by the latter author were used (101).

In a recent review, Huang et al. (2007) showed that central obesity is a key factor in the development of MetS (94). Central obesity leads to dyslipidemia, hyperglycemia and insulin resistance, which in turn may lead to atherosclerosis and peripheral neuropathy. Interestingly, they also suggested that physical inactivity may be an important counteracting factor for the high prevalence of MetS in adolescents. Unfortunately, because of the lack of longitudinal studies there is only weak evidence that physical activity is causally related to the prevalence of MetS. In addition, it is not well understood how diet may affect MetS. Few studies have focused on the effects of a combination of increasing physical activity and improving the quality of the diet on metabolic outcome parameters.

In order to counteract the detrimental health effects of the upcoming pandemic of obesity numerous weight management programs for overweight adults and adolescents have been developed $(46,185)$. Most of these programs include stimulation of a healthy lifestyle through physical activity and healthy diet on the one hand, and behavioral modification at 
the other hand $(110,185)$. Best results were reached in programs were behavioral change was part of the program (110). Additionally, participation of parents during the program has been shown to have positive effects on the outcome of the program (110).

Most of the programs described in the literature were organized and developed in and around a research setting. However, practice-based programs often lack standardization and are less controlled and mostly dictated by practical location and setting. In the present study a practice based lifestyle program Realfit has been evaluated. Realfit is a twelve week program designed for overweight adolescents. The program focuses on weight loss and sports participation and aims to assist adolescents in developing a healthier lifestyle. However, the effectiveness has not been established yet. Therefore, the aim of this study was to evaluate the short and long-term effects of a practice based program Realfit on body composition, MetS, aerobic test performance, and physical activity in adolescents.

\section{METHODS}

\section{Study population and design}

The adolescents participating in the three Realfit programs between September 2005 and June $2006(n=36)$ were invited to participate in the evaluation study. All participants that participated in the group intervention programs were overweight or obese (41). Each intervention group consisted of 12 participants. Four of the participants dropped out during the program. From the 32 remaining participants (15 boys, 17 girls) 7 decided not to participate in the blood collection, but they all agreed to participate in the other measurements. Outcome parameters were measured at baseline, directly after the 12 week program and five months later. All subjects were informed about the nature and risks of the study before and gave their written informed consent to participate. The study was approved by the local medical ethical committee (Maastricht). All measurements were executed in compliance with the Helsinki Declaration.

Besides the intervention group ten adolescents in a control group have been investigated as well (133). The control group consisted of children on a waiting list for the Realfit intervention in 2005. In the control group body height and weight were measured from which BMI was calculated. 


\section{Intervention by the Realfit program}

Realfit is a 12 week intervention program intended for 12 to 18 years old overweight adolescents. The program is organized in several cities in the southern part of the Netherlands, while in this study the program applied in Maastricht was investigated. The aim of the program is to stimulate a healthy life style, healthy nutrition and physical activity. After an information session all interested adolescents are allowed to participate after paying 45 euros. During the program the participants followed a diverse fitness program once a week. There were seven individual consultations with a dietician specialized in adolescents (one intake, five consultations, one closing session). There were three group sessions with the dietician. The parents attended one group session with the dietician and two group sessions with a dietician and a pedagogue.

\section{Outcome parameters}

Anthropometry. To calculate the body mass index (BMI), height and body weight (BW) were determined bare footed in light clothes. Biceps, triceps, sub scapular and crista skin fold thickness was measured in triplicate (Holtain Ltd., Crymych, U.K.). Fat percentage was assessed from the sum of the four skin folds, according to Weststrate and Deurenberg (214). Fat-free mass (FFM) was calculated as '(1-fraction of FM)*BW'. Waist circumference was measured at the narrowest point below the ribs or halfway between the lowest ribs and the iliac crests. Hip circumference was measured the level of the anterior superior iliac spine, but when it could not be felt, at the broadest circumference below the waist. Waist/hip ratio was calculated as waist circumference divided by hip circumference.

Metabolic syndrome. The criteria for the MetS are met when at least three of the following criteria apply: increased waist circumference, increased systolic of diastolic blood pressure, decreased HDL concentrations, increasing triglyceride levels and increased glucose levels, all according to age specific cut-points as defined by Jolliffe and Janssen (2007) (101).Waist circumference has been measured as described above. Blood pressure was measured non-invasively in a seated position on the right arm with the Omron HEM-711AC Blood Pressure Monitor with an appropriate cuff (9). The participants were asked to sit quietly for five minutes before each measurement. The average of two measurements has been used for the analyses. After an overnight fast, blood was collected from a forearm vein and distributed over EDTA and serum tubes for obtaining serum and plasma. The tubes were centrifuged for 10 minutes. Serum and plasma were stored in small tubes at $-80^{\circ} \mathrm{C}$ until analysis. In the samples glucose, insulin, 
HDL cholesterol concentration and triglyceride concentrations were measured. Plasma Glucose (Glucose/HK,), Serum insulin (Insulin) serum $\mathrm{HDL}$ cholesterol (HDL-cholesterol, no pretreatment) and serum triacylglycerol (GPO-PAP) kits were all obtained from Roche Diagnostics, Basel, Switzerland. The concentrations were analyzed with the Roche Hitachi modular analyzer.

Insulin resistance. Additionally, insulin resistance was determined with the homeostasis model assessment for insulin resistance (HOMA-IR)(20), which has been shown to be valid in an overweight population (12) and was calculated from:

Fasting Insulin $(\mu \mathrm{U} / \mathrm{mL}) \times$ Fasting Glucose $(\mathrm{mmol} / \mathrm{L}) / 22.5(123)$.

Aerobic test performance. Aerobic test performance was assessed by the Astrand-Rhyming test and the results were expressed as maximal oxygen uptake (VO2max) (11). The test was performed on a cycle ergometer, at a pedaling rate of $70 \mathrm{rpm}$. The workload was adjusted to obtain a stable heart rate between 130 and $160 \mathrm{bpm}$. According to the test instructions, steadystate is reached when the difference in heart rate after five and six minutes is less than five bpm. If not, the test was continued until steady-state was reached. The maximal oxygen uptake was estimated from a nomogram based on sex, workload, and mean steady-state heart rate during exercise. A correction factor for age was used, and maximum oxygen uptake was expressed in $\mathrm{mL} / \mathrm{kg} / \mathrm{min}$.

Physical activity. Together with the researcher the adolescents filled out the SQUASH questionnaire (212). The SQUASH list investigates physical activity behavior during commuting activities, leisure time activities, household activities, and activities at work and school. The participants were asked to refer to an average week in the past months. Outcome parameters used were; time spent on physical activity, time spent in inactivity and time sport in sports, all expressed in minutes per week.

\section{Statistical analysis}

Results are shown as means ( \pm S.D.). Differences between the three measurements were calculated with the General Linear Model approach for repeated measures. When Mauchly's test indicated that the assumption of sphericity was violated, the degrees of freedom were corrected using Greenhouse-Geisser corrections. Pair wise comparisons for the main effects were corrected using a Bonferroni adjustment.

The comparison of the intervention group and the control group were evaluated by the calculation of change scores for body weight and BMI 
before and after the intervention. The differences between change scores in the intervention and control group were tested with independent t-tests.

To evaluate possible predictors for the success of the intervention, the group was divided into two subgroups. In the first subgroup the BMI decreased during the program and in the second subgroup the BMI increased during the program. An independent t-test was used to test baseline differences between these groups.

Ultimately, General Linear Models for repeated measures with a between subjects component were used to test the differences in the development of characteristics of the MetS between the subgroup who increased and the subgroup who decreased the BMI. For all tests $p<0.05$ is considered statistically significant.

\section{RESULTS}

The study population consisted of 32 adolescents ( 15 boys and 17 girls). The average age at baseline was $14.68( \pm 1.67)$ years. The average BMI at baseline was $29.19 \mathrm{~kg} / \mathrm{m}^{2}( \pm 4.10)$. The other baseline characteristics are shown table 1 (T0). This table also shows the characteristics at T1 (directly after the 12 week program) and T2 (5 months after the end of the program), and the $p$-value for the general linear model for repeated measures.

The results show that height, BW, BMI, hip circumference and systolic and diastolic blood pressure were significantly different between the moments of testing. Pair wise comparison showed that differences between T0 and T1 and between $\mathrm{T} 1$ and $\mathrm{T} 2$ were significant for BW. For BMI, hip circumference, and systolic and diastolic blood pressure only the development from T0 to T1 reached to level of significance. There was a trend in VO2max and time spent on physical inactivity. All other measured variables did not change significantly during and after the intervention period in the total intervention group.

When the prevalence of the MetS was evaluated, 10 out of the 25 participants (in which blood was collected), could be classified as having the MetS before the intervention started. Directly after the intervention 7 adolescents met the criteria for the MetS, and five months later, five adolescents again met the criteria of the MetS.

In the control group, body weight and BMI did not change significantly $(p>$ 0.05) during the time of the intervention. When these results were compared to the intervention group, it was shown that the BMI change during the 12 week intervention was $-0.85 \mathrm{~kg} / \mathrm{m}^{2}$ in the intervention group, whereas in the control group the change in $\mathrm{BMI}$ during the intervention was 


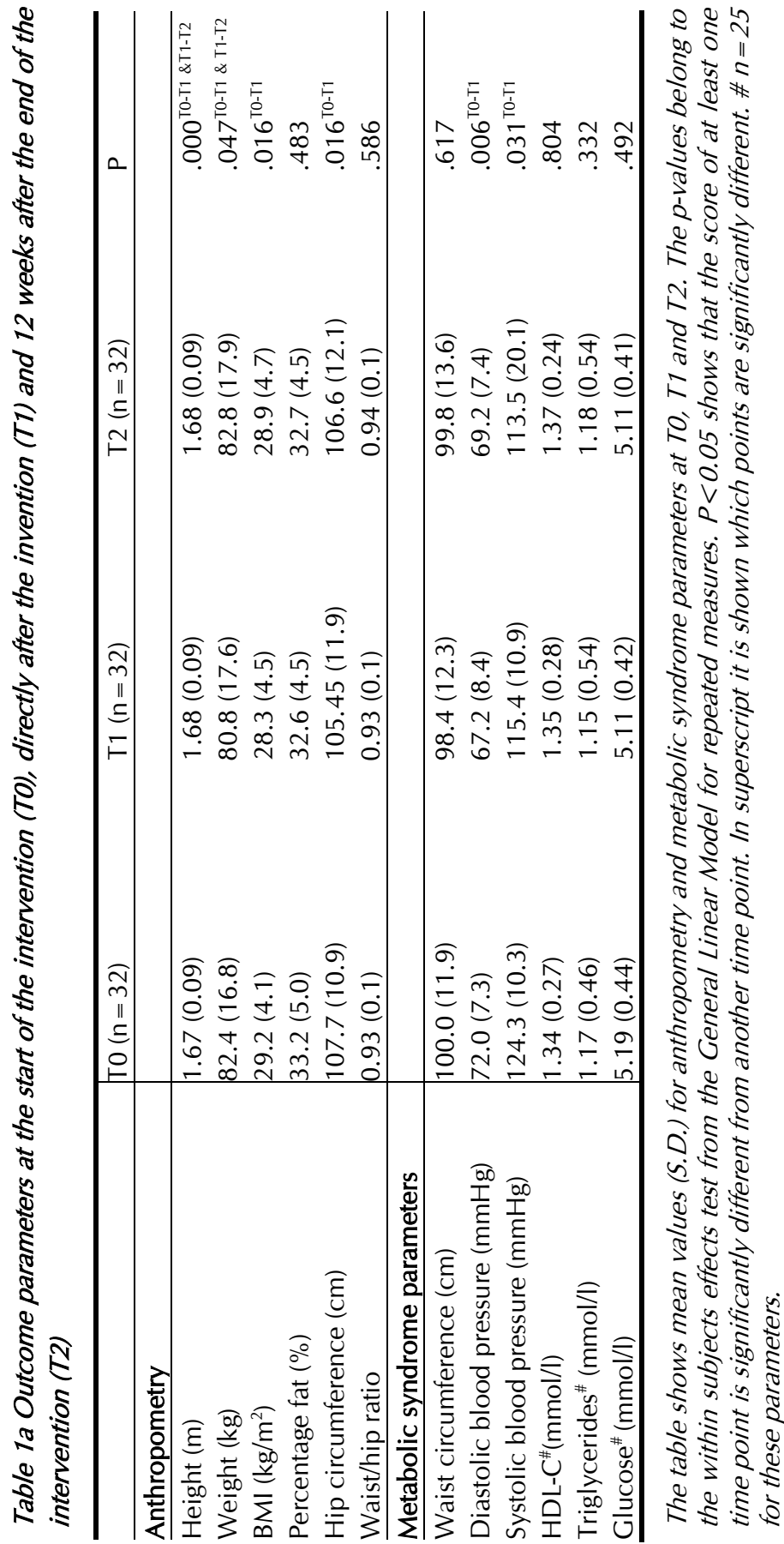




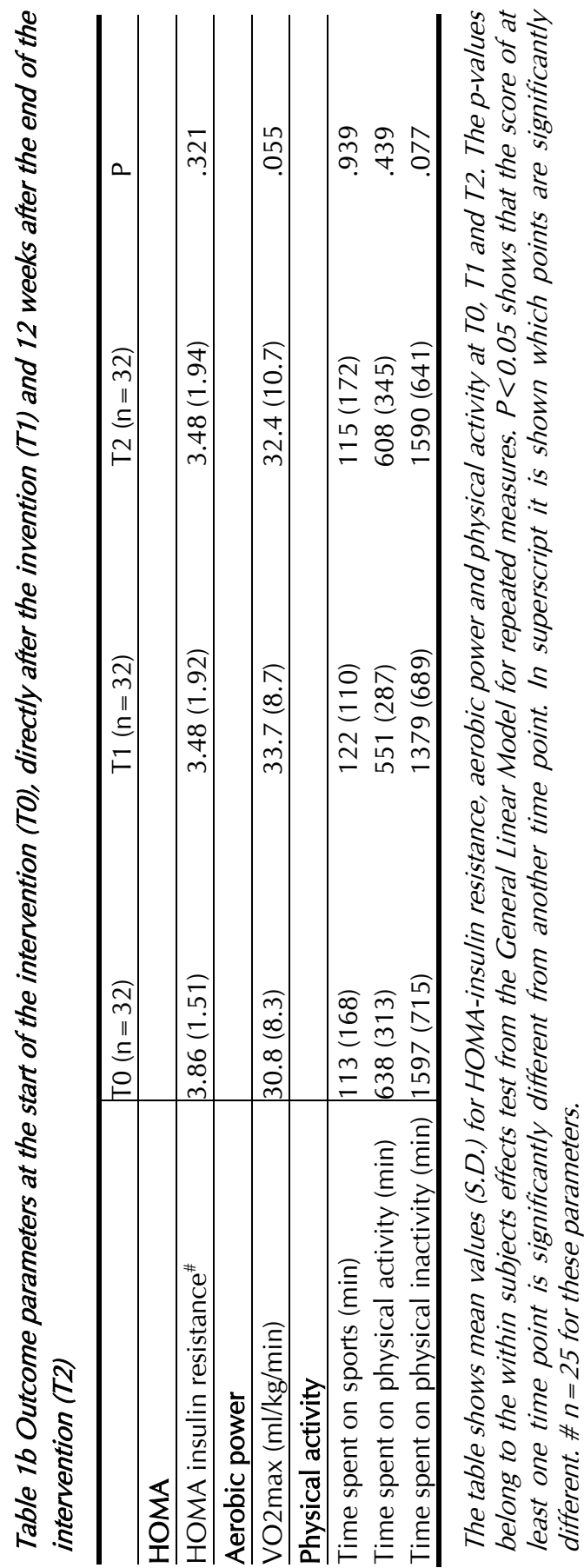


$-0.02 \mathrm{~kg} / \mathrm{m}^{2}$. The difference in BMl change scores was statistically significant $(p=0.048)$. Body weight increased with $1.31 \mathrm{~kg}$ in the control group, whereas the intervention group lost $1.56 \mathrm{~kg}(p=0.011$ for the difference between both groups). Baseline values for children who were able to decrease their BMI during the program $(n=25)$ were compared to the children who's BMI increased during the program $(n=7)$ in table 2. It was found that the participants who decreased their BMI had a significant lower $\mathrm{BMI}$, body weight and hip circumference at baseline compared to the participants who gained BMI during the intervention. After the intervention period, mean BMI increased in both subgroups, although these increases did not reach the level of statistical significance in both subgroups.

Table 2 Predictors for a decrease in BMI during the program

\begin{tabular}{llll}
\hline & $\begin{array}{l}\text { BMl increased } \\
(\mathrm{N}=7)\end{array}$ & $\begin{array}{l}\text { BMI decreased } \\
(\mathrm{N}=25)\end{array}$ & $\mathrm{P}$ \\
\hline BMI before intervention $\left(\mathrm{kg} / \mathrm{m}^{2}\right)$ & $32.1(4.5)$ & $28.4(3.7)$ & .030 \\
Body weight $(\mathrm{kg})$ & $94.8(19.2)$ & $78.9(14.7)$ & .024 \\
Hip circumference $(\mathrm{cm})$ & $117.1(15.1)$ & $105.1(7.9)$ & .008 \\
\hline
\end{tabular}

Parameters that showed significant differences at baseline between the group who decreased and increased the BMI during the program. There were no significant differences with regard to height, percentage fat, waist circumference.

Table 3 shows the results of the General Linear Model for repeated measures test in which differences in the development of the characteristics of the MetS and HOMA-IR and VO2max were tested for the group who decreased or increased the BMI during the program.

Only HOMA-IR had a significantly different pattern over time in the two groups. During the program insulin resistance was increasing in the subgroup that increased their BMI and decreased in the subgroup that decreased their BMI. Interestingly, after the end of the program, insulin resistance decreased again in the subgroup that increased the BMI during the program. 
REALFIT: A LIFESTYLE INTERVENTION FOR OVERWEIGHT ADOLESCENTS

Table 3 Comparison between successful or unsuccessful participation

\begin{tabular}{ll|llll}
\hline & BMI & T0 & T1 & T2 & P \\
\hline Diastolic blood pressure & $\uparrow$ & $75.4(7.1)$ & $67.1(6.9)$ & $73.1(8.1)$ & .252 \\
(mmHg) & $\downarrow$ & $70.9(7.5)$ & $67.2(8.1)$ & $68.0(7.8)$ & \\
Systolic blood pressure & $\uparrow$ & $125.9(12.1)$ & $117.4(10.9)$ & $104.3(15.2)$ & .173 \\
(mmHg) & $\downarrow$ & $123.8(13.8)$ & $114.7(10.8)$ & $116.4(16.4)$ & \\
Glucose $^{\#}(\mathrm{mmol} / \mathrm{l})$ & $\uparrow$ & $5.2(0.5)$ & $5.2(0.5)$ & $5.2(0.5)$ & .839 \\
& $\downarrow$ & $5.2(0.4)$ & $5.1(0.4)$ & $5.1(0.4)$ & \\
HDL-C $^{\#}(\mathrm{mmol} / \mathrm{l})$ & $\uparrow$ & $1.3(0.3)$ & $1.3(0.3)$ & $1.4(0.2)$ & .986 \\
& $\downarrow$ & $1.3(0.3)$ & $1.4(0.4)$ & $1.4(0.3)$ & \\
Triglycerides ${ }^{\#}(\mathrm{mmol} / \mathrm{l})$ & $\uparrow$ & $1.4(0.5)$ & $1.4(0.5)$ & $1.3(0.6)$ & .639 \\
& $\downarrow$ & $1.1(0.4)$ & $1.0(0.3)$ & $1.1(0.4)$ & \\
HOMA insulin resistance & $\uparrow$ & $4.2(2.0)$ & $4.8(2.2)$ & $3.7(1.2)$ & $.032^{\text {T0-T1 }}$ \\
& $\downarrow$ & $3.7(1.2)$ & $2.9(1.2)$ & $3.4(1.9)$ & \\
VO2max (ml/kg/min) & $\uparrow$ & $2.3(0.6)$ & $2.5(0.6)$ & $2.1(0.8)$ & .129 \\
& $\downarrow$ & $2.6(0.8)$ & $2.7(0.9)$ & $2.7(0.8)$ & \\
\hline
\end{tabular}

Development in characteristics of the MetS, HOMA for insulin resistance and VO2max in the groups who decreased $(\downarrow)$ and increased $(\uparrow)$ BMI during the program. The participants who decreased their BMI during the program were called successful, whereas the participants who increased their BMI were called unsuccessful. P-values are shown for the interaction between group and time.

\section{DISCUSSION}

The results of the present study show an overall short-term reduction of BW, $\mathrm{BMI}$, waist circumference and systolic and diastolic blood pressure and a slight reduction in the number of subjects with MetS in a group of overweight adolescents participating in Realfit. On the long-term most results are reversed. Comparing the intervention group with the control group, a significant decrease in BMI and body weight was observed in the subjects who participated in the Realfit program.

The overall results of the Realfit program are in line with the results of other short-term weight management programs. For instance, Eliakim et al. (2002) reported similar decreases in $\mathrm{BMI}$ and body weight using a slightly different 12 week program (63). In the current study in $25(78 \%)$ adolescents out of the intervention group BMI decreased significantly during the 12 week program, whereas in seven adolescents BMI increased during the program. In retrospective, an interesting finding of the present study was a difference in baseline anthropometric variables between the participants who increased their BMI (unsuccessful participants) and those who decreased the 
BMI during the program (successful participants). The unsuccessful subjects started with a higher BMI, body weight, and waist- and hip circumference. It is tempting to speculate that adolescents with a higher degree of overweight have more difficulty to reduce the level of overweight. These findings are in contrast with the findings of Eliakim et al. (2002) who reported that neither body weight, nor degree of obesity had an influence on BMI changes (63), and with the data published by Braet et al. (2006) who reported that in children, higher baseline adjusted BMI, and higher age are predictors of a successful program (25). In addition, Reinehr et al. (2003) showed that the only significant predictor of success for the treatment of obese children and adolescents was previous participation in exercise groups (151). At present there is no explanation for the discrepancy in these findings although it is possible that children with a high level of overweight have different personality characteristics, and may react differently to various programs (184, 189). Since statements about success predictors in the adolescent population are conflicting, more research is needed to unravel this complex interplay between successful and unsuccessful overweight/obese adolescents. It may be possible to increase the success rate by selection of potential participants based on the baseline level of overweight and/or possible other factors, such as personality characteristics.

The decrease in body weight, BMI, hip circumference and blood pressure in the participants was reversed 20 weeks after the end of the program. Such a regain in weight is not uncommon and is referred to as weight cycling (127, 135). A relapse to pre-intervention levels was also shown for insulin resistance. It was revealed that a population based program like Realfit is able to decrease the insulin resistance of the successful participants on the short-term. This is an important finding because type 2 DM and insulin resistance are the most frequently mentioned consequences of overweight (52). Unfortunately, five months after the end of the program, the participants were unable to maintain the decrease in insulin resistance. An explanation for this relapse is the absence of a proper follow-up program. After the current program, the participants do not meet as a group any more and only see the dietician sporadically. The duration of the current program is too short to be able to individually apply the proposed new lifestyle after the program. This emphasizes the importance of long-term follow-up supervision, as it has also been emphasized in a recent meta-analysis on effective weigh loss in overweight children (175). The follow-up could exsist of monthly group meetings under supervision of the dietican and should continue for at least one year, because it is known that adults who maintain weight loss for 1 year are more likely to continue (177).

One of the aims of the present study was to investigate whether the factors belonging to the MetS do change during the program. In the total group, 
blood pressure was the only characteristic of the MetS which changed significantly, the other characteristics did not change. Ten out of the 25 subjects $(40 \%)$ who participated in blood collection met the criteria of the MetS at the start of the program. This prevalence of MetS is in accordance with recent published data that reported MetS in 30-60\% in overweight children $(44,77,208)$. Immediately after the termination of the program the prevalence was reduced to $28 \%$ ( 7 out of 25 subjects). Despite the regain in weight and $\mathrm{BMI}, 20$ weeks after termination of the lifestyle program there was no increase in the prevalence of MetS (40 vs. 28 vs. $20 \%$ for preimmediately post and 20 weeks post program, respectively). This may seem contradictory but is probably explained by the finding that in most overweight adolescents the values of the parameters defining the MetS were just above the cut-off levels. To the best of our knowledge this is the first study that showed a reduction in the prevalence of the MetS after a practice based lifestyle intervention program.

Although the effects of comparable programs described in the literature are inconsistent, some factors may contribute to the success of a program. One is caloric intake during the intervention, and in some successful programs a maximal caloric intake of $1200 \mathrm{kcal}$ has been applied. Although very low caloric programs are successful in weight reduction, it has been questioned whether these programs are appropriate in growing children (63). The latter authors suggested that in the majority of overweight adolescents, weight maintenance rather than weight loss should be the major goal. This would imply that the focus of intervention programs for overweight adolescents should be shifted from diet to physical activity. Since more emphasis on physical activity will contribute to maintain muscle mass as well as function, which additionally may have impact on health (174). It also appears that successful practice based programs for childhood overweight and obesity require a multi-disciplinary approach and including lifestyle interventions, behavioral modifications and parental participation (218). In the program described in the present study the attention for and emphasize on these items was limited, with only three parental sessions and three group sessions about lifestyle changes. This may explain why in the Realfit program BMI regain was found five months after termination of the program.

On the short-term a tendency for increase in VO2max using a cycle ergometer Åstrand-Rhyming test was shown, although the increase did not reach the level of statistical significance $(p=0.055)$. This is in contrast with the results of Eliakim et al. (2002) who reported a significant increase in physical exercise capacity (endurance time during a treadmill test). However, exercise training in the program of Eliakim et al. (2002) was a major focus, whereas the Realfit program mainly focused on weight loss 
established through lifestyle changes. The present study showed that the time spent on physical activity and the time spent on sports was not significantly affected by Realfit.

In conclusion, it has been demonstrated that a practice based lifestyle program improved body composition and blood pressure on the short- term. Moreover the prevalence of the MetS decreased. Participants with a lower $\mathrm{BMI}$ at baseline seemed to be more responsive to the program. Successful participants also decreased their insulin resistance on the short-term. Most results were reversed on the long-term. Although some short-term beneficial effects of the program were found, long-term results may be further improved by adjustments to the program and long-term follow up.

\section{ACKNOWLEDGEMENTS}

We gratefully thank Sandra Mulkens for her assistance regarding the data of the control group. 


\section{CHAPTER 8}

General Discussion 


\section{General Discussion}

The main aim of the studies described in this thesis was to investigate associations between body composition (fat mass and fat free mass), aerobic fitness and insulin resistance in Dutch adolescents living in the province of Limburg. The background for this scientific interest comes from the worldwide growing prevalence of insulin resistance and type 2 diabetes (14, 217). Not only in adult life but also in childhood and adolescence do these health problems increase $(57,66,108,119,145,146)$. The most frequently reported underlying risk factors for developing insulin resistance and type 2 DM are overweight and/or obesity $(8,111,152,209)$. Because the prevalence of overweight has also been increasing rapidly in adolescents (96), a further rise in the prevalence of insulin resistance and type 2 DM can be expected. Finally, several researchers have pointed out that nowadays young people also spend less time on physical activities and sports $(54,61)$, which may increase the risk factors for insulin resistance and type 2 DM. Taking these alarming prevalence numbers into consideration, it is of scientific and societal interest to investigate how this upcoming epidemic can be stopped and preferably be prevented (13). Therefore, in this thesis the possible associations between insulin resistance, body composition and aerobic test performance have been studied in adolescents.

\section{Body composition}

Applying age-adjusted cut-off values for BMI as described by Cole et al. (2000) (41) in the adolescents that participated in the study as described in Chapter $4,13 \%$ could be classified as overweight and $4 \%$ as obese. This confirms data from a recent study in a comparable Dutch population (96). Although it is regularly reported in the media that nowadays one out of every eight children in the Netherlands is overweight (204), most recent studies $(96,195)$ showed that this prevalence number is currently exceeded in the adolescent group. In fact, an estimate of one overweight or obese individual in every six adolescents is realistic and thus emphasizes the importance of monitoring the prevalence regularly, because of its (negative) impact on health.

One has to bear in mind, however, that the interpretation of the BMI as parameter for overweight is more complex than it may seem at first sight $(72,210)$. Since obesity is considered as an excessive fat accumulation and the $\mathrm{BMI}$ concerns the total body weight, the BMI may not always give accurate information about the fat content of the body (72). For instance, 
when simply using BMI as an indicator for overweight, a higher number of adolescents who actually fail to have large fat stores are classified as overweight or obese. Moreover, it has been shown that $32 \%$ of females and $42 \%$ of males classified as obese by BMI did not have high adiposity or fat mass (156) and vice versa. In order to overcome misinterpretations when using the BMI method, fat percentage should be measured as an alternative or additional method (210). In population based studies the sum of skin fold thickness can be used to estimate percentage fat (214). Both BMI and percentage fat can subsequently be used for the calculation of, yet uncommon but more reliable Fat Mass Index (FMI) and Fat Free Mass Index (FFMI), as described in Chapter 5 of this thesis $(210,211)$. In brief, a person with a relatively high FFMI compared to the FMI (a solid person, see Chapter 5) is assumed to be healthier compared to a person with relatively small FFMI (a slender person). We, and others $(174,210)$ suggest that in future studies researchers use these combined parameters rather than using $\mathrm{BMI}$ and percentage fat alone.

Although this proposed method gives more accurate information about body build, in practice its use may not be trouble-free. At present there are only few health care workers who are properly instructed to perform skin fold measurements with the required accuracy. An alternative and promising measure may be found in the waist and hip circumference measurements $(92,156)$. These measures are not only easy to apply, but the circumferences may also be more directly associated with health risks (116), because the distribution of fat over the mid-waist of the body is associated with health as well $(125,130)$. Independent of the method used, age and gender specific cut-off values should be available and be used accordingly (41). At present there are no specific cut-off values for waist circumference for children and/or adolescents. This is one of the relevant issues which should be addressed in future studies.

In conclusion, although the BMI measurement should be interpreted with caution, BMI data show increasing numbers of overweight and obesity in Dutch children and adolescents. This emphasizes the importance of monitoring this development, and for more accurate results, overweight should preferably be monitored with enhanced methods.

\section{Metabolic derangements}

In $15 \%$ of a Dutch adolescent population a fasting whole blood capillary glucose concentration above the cut-off value of $5.6 \mathrm{mmol} / \mathrm{l}$ was found (Chapter 4). The current study was comparable to a study in 'healthy' 
American adolescents where $11 \%$ was found to have an elevated fasting glucose concentration (55). This suggests that the incidence of elevated fasting glucose levels in Dutch children exceeds that of their American counterparts. However, it has to be taken into account that the prevalence numbers in both studies may have been overestimated, because the data were obtained from a single measurement. In case of an elevated fasting glucose level the measurement should be repeated on another day (74). Therefore, in contrast to the study in American adolescents, in the current study the measurement was repeated in subjects with an initial elevated fasting glucose level. Extrapolating the confirmed prevalence to the present population, it is estimated that about $3 \%$ of the healthy adolescent population has a confirmed elevated fasting glucose concentration, which is fairly lower than the American estimation (55). Since it is likely that not all children were truly fasted during the first measurement in both studies, the current estimation of $3 \%$ after confirmation seems to be closer to reality. Therefore, at this stage, large scale screening for insulin resistance in the Dutch adolescents does not appear to be appropriate. However, the prevalence should regularly be monitored in high risk groups, in order to enable adequate action as quickly as possible.

Some risk factors have been shown to increase the susceptibility for impaired glucose tolerance, which is the first stage of insulin resistance. In the group described in Chapter 4 educational level was for the first time identified as risk factor for impaired glucose concentrations. The prevalence of elevated glucose concentration was significantly higher in the students enrolled at pre-vocational school level (VMBO; Voorbereidend Middelbaar Beroeps Onderwijs) compared to the students following pre-university education (HAVO; Hoger Algemeen Voortgezet Onderwijs/VWO; Voorbereidend Wetenschappelijk Onderwijs). This indicates that prevention programs should specifically focus on children participating in the lower Dutch education level. Since in the same chapter it was shown that a higher $\mathrm{BMI}$ is a risk factor for insulin resistance as well, prevention activities should also be targeted at overweight and obese adolescents. In the overweight population (Chapter 7 ) an alarming $40 \%$ of the children met the criteria which describe the metabolic syndrome as described by Jolliffe and Janssen (2007) (101). This, once again, emphasizes the importance of prevention programs for overweight/obese children.

In Chapter 3 it was shown that a positive family history for diabetes was a significant risk factor for insulin resistance at the age of 12 . At the age of 7 (Chapter 2$)$ this association was close to significance $(p=0.07)(173)$. This confirms previously published studies that showed that the risk for insulin resistance is higher in children with a positive family history for type 2 diabetes $(8,82)$. Consequently, health care professionals treating diabetic 
patients should be aware of an increased risk in the offspring of their patients, especially when other risk factors (overweight and lower educational level) are present as well.

Pubertal development has also been addressed as a risk factor for insulin resistance (8). However, this may be biased by normal growth and development since during puberty a temporary change in insulin resistance may occur. In the present study, also an increase in insulin resistance from the age of 7 to 12 was shown (Chapter 3). However, in smaller previous studies it has been shown that the increase in insulin resistance during puberty was only temporary, and returned to pre-pubertal levels after puberty $(16,81)$. When a change in insulin resistance can be confirmed in a substantial longitudinal study as being transient, puberty can be excluded as a risk factor for persistent insulin resistance. Besides this, after confirmation it may not be appropriate to use the same cut-off values across different pubertal stages. Therefore, in future studies specific attention should be paid to the definition of impaired glucose tolerance and insulin resistance in adolescents in various stages of puberty. Finally, in chapter 3 tracking of insulin resistance between the age 7 and 13 has been shown to be significant. In future studies it should be further investigated at what age insulin resistance is predictive for the development of type $2 \mathrm{DM}$.

In conclusion, prevention programs for insulin resistance in youngsters should focus on boys and girls at lower educational levels, with a positive family history, and with overweight or obesity.

\section{Physical fitness, body composition and health}

In the current thesis it was shown that physical fitness was not directly associated with insulin resistance, whereas in previous studies in subjects with a more unhealthy body composition a significant association could be shown $(4,98)$. These studies suggested that in an overweight population, physical activity and fitness may reduce the negative effects of being overweight on health (134). In the relatively healthy populations described in this thesis body composition was significantly associated with insulin resistance, but the tempering effect of physical fitness could not be shown.

Since physical fitness has been shown to be independently associated with body composition (Chapter 5), physical activity and fitness may be considered as factors that can prevent an unfavorable body composition. Body composition is mainly defined by two main compartments the fat mass and the fat free mass. Unfortunately, the focus is generally on the fat mass, while the fat free mass is often disregarded (216). The fat mass seems 
to be involved in the association by means of it production of adipokines like leptin and adiponectin $(8,173,178)$. From a health perspective, a highquality fat free mass is at least as important as a limited fat mass, because the fat free mass consists for a large part of muscle mass, which is not only one of the largest tissues in size and plays a pivotal role in the clearance of glucose and fat (171). It has been shown in adolescents that physical activity can on the one hand decrease the fat mass and on the other hand increase the fat free mass $(51,76,86)$. Either way, both a lower fat mass as well as a higher fat free mass may decrease the chances to develop insulin resistance, which suggests that physical activity and fitness may indirectly prevent children and adolescents from developing insulin resistance. Future studies should investigate the association between physical fitness and insulin resistance and focus not only on the fat mass but also on the fat free mass and the physical activity level.

Although there is a strong inverse association between fitness and body weight in adolescents, it is still unknown how fitness levels can be improved most effectively (23). Since physical fitness appears to be an important factor for preventing overweight and insulin resistance its assessment needs further scientific attention. Scientific studies have used diverse test protocols and methodologies which make comparisons between studies complex and difficult. In the present study aerobic capacity, as measured with a cycle ergometer test or a shuttle run test, was compared to data collected previously in the Dutch population in 1985 and 1987 (Chapter 6) $(84,198)$. When the test results were normalized to body weight a $13 \%$ lower aerobic capacity $(\mathrm{W} / \mathrm{kg})$ was found in the adolescents in the current study in comparison to the participants of the previous study (84). This decrease in fitness level normalised to body weight is more likely due to increases in body fat in youth, rather than deterioration in cardiovascular function (159). The current comparison was also complicated by differences in the methods used. This emphasizes that in future studies it is important to use standardized methods to measure performance capacity. For testing in a research setting cycle ergometers seem to be appropriate and convenient, but the used protocol should be standardised. For testing in a field situation in larger groups, the types of tests are even more variable which especially requires global standardization for field tests. We suggest using the shuttle run test as a field test and a cycle ergometer test as the standard test in the research setting. An expert panel should agree on the types of tests and the protocol which could be used best.

In addition to the former recommendation, the decrease in physical fitness level, which was in line with previous international studies $(139,192)$ emphasised the importance of the stimulation of an active lifestyle in Dutch adolescents and the regular monitoring of physical fitness and activity level. 
The current activity advice (one hour of physical activity a day) might be appropriate, but the environment does not seem to trigger adolescents to be active, which results in an average of $25 \%$ of the adolescents accomplishing this norm (166). This suggests that more attention should be paid to advices how to achieve an hour of physical activity a day and more efforts should be put in creating an activity friendly environment.

\section{Intervention and prevention}

Numerous intervention programs aim to decrease the level of overweight and obesity in children and adolescents. In Chapter 7 the results of one of these practice-based programs (Realfit) are described. It was shown that Realfit has some favorable effects on health related variables on the shortterm, whereas most of the positive effects were reversed on a longer term. Based on this it is postulated that the most important focus of any intervention program should be long-term maintenance of a healthy life style. To achieve this goal some requirements should be met: a) a low threshold for entering the program; b) participation should be fun; c) nutritional and physical activity information should be provided; d) the physical part should include endurance as well as resistance activities; e) to stimulate the group feeling weekly meetings should be organized for teaching a healthy lifestyle, and f) there should be long-term supervision. The last requirement is that the aims of the participant should match the aims of the program organizers. In Realfit the main aim of the program organizers was to teach the participants a healthy life style, whereas for the participants the most important reason for participation was weight loss and improvement of physical appearance. Fixing this mismatch is a major challenge in designing intervention programs.

Organizing a program that meets all the above mentioned requirements is expensive and even in the best organized programs it is most likely that some participants will drop out. Regarding success, pre-intervention body weight is also a predictive factor for success, because it was shown (Chapter 7) that a program like Realfit appears to be less effective in participants who started with a higher level of overweight. All these points emphasize the need to focus on overweight prevention rather than on overweight reduction. Prevention should aim at adopting a healthy life style, including healthy nutrition and sufficient physical activity. In 1998, the Dutch norm for health enhancing physical activity (NNGB; Nederlandse Norm Gezond Bewegen) was established based on the American standard (143), 
which recommends children to be moderately physically active for at least one hour a day. Unfortunately, according to Statistics Netherlands (CBS; Centraal Bureau voor de Statitiek) only $25 \%$ of the 12 to 18 year old adolescents was able to meet this norm in 2004 (166). This low number of sufficiently active adolescents in combination with the upcoming obesity epidemic, indicate that in prevention activities more emphasis should be placed on physical activity. One of the effective strategies may be to organize at least one daily hour of physical activity during school hours. This physical activity session can either be integrated in the school program or being offered as an extracurricular activity. Extra attention should be paid to active participation during these lessons, especially concerning the overweight children, because especially this group tends not to participate in an active way. To further develop such a strategy, political coordination and government support are needed.

Eucation is one of the tools to stimulate a healthy lifestyle,. In education activities it is important to include information about the risks associated with an unhealthy lifestyle. There appears to be a lack of knowledge in this field, which is nicely illustrated in a study from The Netherlands Nutrition Centre (Voedingscentrum) (202), where only 13\% of the people named diabetes as a possible consequence of overweight or obesity. Therefore, due to the upcoming pandemic of diabetes more emphasis should be put on early signs of the disease and on education about prevention strategies. It has been shown that educational programs have a low effectiveness when motivation of participants is low. Motivating people to participate in prevention programs especially the target groups is one of the major challenges for reversing the alarming trend for an unhealthy life style.

Although effective programs should be organized to decrease the level of overweight in affected children; priority should be given to the prevention of the problem. Physical activity should be considered as an important tool for prevention. In conclusion, there is urgent need that those responsible for young people's health, including parents, schools and practice health providers, emphasize the importance of effective interventions and encourage increased physical activity levels (106).

\section{Methodological considerations}

In depth knowledge of health related concepts is always important. It is even more important during childhood, because this is a crucial period in the (pre)development of certain disorders/diseases but it is also the period during which the foundations for a healthy lifestyle can be laid (176). It has 
to be noticed, though, that scientific studies in adolescents are not easy to undertake because adolescents may feel vulnerable and are less motivated than individuals of other age groups to participate in research. Reasons for non-participation are often a busy schedule, and another possible factor is that when studies involve physical examinations, some adolescents feel embarrassed. Finally, a large percentage of children and adolescents hesitate to participate in studies when blood collection is included. These issues result in a low participation rate and in future studies specific attention should be paid to increase motivation and to recruit participants in this age category. It has to be realized that most scientific studies that have included human volunteers as subjects, may have selection bias. Persons participating are usually preoccupied with the benefits of health intervention.

Additionally, in studies in children and adolescents the practical feasibility is important. Most standard techniques, like the hyperinsulinemic, euglycemic insulin clamp technique to measure insulin resistance are highly invasive and complex which makes them not easy to use in population based studies in adolescents $(107,129)$. In order to keep the response rate as high as possible in this group one should try to minimize the burden on the participants (129). In the study described in Chapter 4, it was decided to estimate insulin resistance by measuring fasting glucose concentration, by using the capillary blood glucose measurement. Even with this less invasive method the response rate was only $30 \%$. This left us and leaves many scientists, with a dilemma regarding the choice between more reliable and invasive methods or less reliable but also less invasive methods. For example, a venous puncture would result in more reliable results but the response rates are low or the capillary method results are less reliable data but response rates are higher.

Finally, although scientists are usually confronted with more methodological difficulties in studies with children and adolescents when compared to studies in adults, the results of these studies in youngsters are important because conclusions drawn from studies in adults cannot be extrapolated to children and adolescents.

\section{Recommendations}

The recommendations listed below are divided into recommendations for research purposes and recommendations for practical use. 


\section{Recommendations for research}

- The focus within obesity research should shift from intervention to prevention.

- More detailed studies regarding prevalence numbers of insulin resistance in adolescents should be undertaken in high risk groups; namely the overweight group, adolescents with a positive family history for diabetes and adolescents attending lower educational levels.

- Thorough information regarding the age at which insulin resistance can reliably predict a positive development of type 2 diabetes at adult age (tracking) is necessary.

- Since insulin resistance seems to increase temporarily during puberty, age specific cut-off values for insulin resistance and type 2 diabetes should be established.

- Besides the fat mass, more attention should be paid to the fat free mass in overweight people, because the positive effects of this type of mass may counteract possible negative effects of the fat mass.

- For the determination of overweight and obesity, waist circumference seems to be a promising method. Therefore, health related cut-off values should be established.

\section{Recommendations for practical use}

- Prevention programs should be evidence based and for that reason continuously evaluated and studied.

- The upcoming obesity pandemic should be monitored regularly, specifically in children and adolescents. Knowledge transfer about the overweight pandemic to the general public as well as to all health professionals should be addressed in order to develop adequate prevention and intervention actions.

- Health care workers should more explicitly focus on insulin resistance and type 2 diabetes. This is especially valid in individuals with one or more of the following characteristics: a positive family history for diabetes, overweight or obesity, and attending lower educational levels. 
- Intervention programs for overweight children and adolescents should aim more explicitly at long-term results (for example with monthly group sessions) and make sure that participants and organisers share the same intentions and aims.

- Also in field testing methods should be standardized, in particular in the determination of overweight as well as in the measurement of insulin resistance and physical fitness. 
REFERENCES 
1. Ahmed ML, Ong KK, Morrell DJ, et al. Longitudinal study of leptin concentrations during puberty: sex differences and relationship to changes in body composition. J Clin Endocrinol Metab 1999; 84:899-905.

2. Ajala MO, Oladipo OO, Fasanmade $\mathrm{O}$, et al. Laboratory assessment of three glucometers. Afr J Med Med Sci 2003; 32:279-82.

3. Allard P, Delvin EE, Paradis G, et al. Distribution of fasting plasma insulin, free fatty acids, and glucose concentrations and of homeostasis model assessment of insulin resistance in a representative sample of Quebec children and adolescents. Clin Chem 2003; 49:644-9.

4. Allen DB, Nemeth BA, Clark RR, et al. Fitness is a stronger predictor of fasting insulin levels than fatness in overweight male middle-school children. J Pediatr 2007; 150:383-7.

5. American-Diabetes-Association. Standards of medical care in diabetes-2006. Diabetes Care 2006; 29 Suppl 1:S4-42.

6. Andersen LB, Harro M, Sardinha LB, et al. Physical activity and clustered cardiovascular risk in children: a cross-sectional study (The European Youth Heart Study). Lancet 2006; 368:299-304.

7. Armstrong N, Welsman JR and Kirby BJ. Peak oxygen uptake and maturation in 12-yr olds. Medicine and Science in Sports and Exercise 1998; 30:165-9.

8. Arslanian S. Type 2 diabetes in children: clinical aspects and risk factors. Horm Res 2002; 57 Suppl 1:19-28.

9. Artigao LM, Llavador JJ, Puras A, et al. [Evaluation and validation of Omron Hem 705 CP and Hem 706/711 monitors for self-measurement of blood pressure]. Aten Primaria 2000; 25:96-102.

10. Arts $\mathrm{F}$, Kuipers, $\mathrm{H}$. The relation between power output, oxygen uptake and heart rate in male atheletes. Int J Sports Med 1994; 15:228-31.

11. Åstrand PO RK Textbook of Work Physiology: Physiological Bases of Exercise (ed 4). ed. I. Champaign, Human Kinetics. 2003.

12. Atabek ME and Pirgon O. Assessment of insulin sensitivity from measurements in fasting state and during an oral glucose tolerance test in obese children. J Pediatr Endocrinol Metab 2007; 20:187-95.

13. Atabek ME, Pirgon $\mathrm{O}$ and Kurtoglu S. Assessment of abnormal glucose homeostasis and insulin resistance in Turkish obese children and adolescents. Diabetes Obes Metab 2007; 9:304-10.

14. Baan C, Poos, M.J.J.D., [What is the prevalence of diabetes mellitus and how many people die from this disease?], in Volksgezondheid Toekomst Verkenning, Nationaal kompas Volksgezondheid., www.nationaalkompas.nl, Editor. 2005, RIVM: Bilthoven.

15. Ball GD, Shaibi GQ, Cruz ML, et al. Insulin sensitivity, cardiorespiratory fitness, and physical activity in overweight Hispanic youth. Obes Res 2004; 12:77-85. 
16. Ball GD, Huang TT, Gower BA, et al. Longitudinal changes in insulin sensitivity, insulin secretion, and beta-cell function during puberty. J Pediatr 2006; 148:16-22.

17. Bemelmans WJW, Hoogenveen, R.T., Visscher, T.L.S., Verschuren, W.M.M., Schuit, A.J., [Future developments in overweight. Estimated effects on public health], RIVM, Editor. 2004: Bilthoven.

18. Bloomgarden ZT. Type 2 diabetes in the young: the evolving epidemic. Diabetes Care 2004; 27:998-1010.

19. Blundell JE, Goodson S and Halford JC. Regulation of appetite: role of leptin in signalling systems for drive and satiety. Int J Obes Relat Metab Disord 2001; 25 Suppl 1:S29-34.

20. Bonora E, Targher G, Alberiche $\mathrm{M}$, et al. Homeostasis model assessment closely mirrors the glucose clamp technique in the assessment of insulin sensitivity: studies in subjects with various degrees of glucose tolerance and insulin sensitivity. Diabetes Care 2000; 23:57-63.

21. Bouchard C, Shephard, R.J., Physical actvity, fitness and health: the model and key concepts, In Physical activity, fitness and health. International Proceedings and Concensus Statement, C. Bouchard, Shephard, R.J., Stephens, T., Editor. 1994, Champaign III: Human Kinetics. p. 77-88.

22. Bouchard C, Shephard, R.J., Stephens, T., Sutton, J.R., McPherson, B.D., Exercise, fitness, and health: The Consensus Statement, In Exercise, fitness, and health. A consensus of current knowledge, C. Bouchard, Shephard, R.J., Stephens, T., Sutton, J.R., McPherson, B.D., Editor. 1990, Human Kinetics Publishers, Inc.: Champaign. p. 5.

23. Bovet $\mathrm{P}$, Auguste $\mathrm{R}$ and Burdette $\mathrm{H}$. Strong inverse association between physical fitness and overweight in adolescents: a large school-based survey. Int J Behav Nutr Phys Act 2007; 4:24.

24. Bradley RH and Corwyn RF. Socioeconomic status and child development. Annu Rev Psychol 2002; 53:371-99.

25. Braet C. Patient characteristics as predictors of weight loss after an obesity treatment for children. Obesity (Silver Spring) 2006; 14:148-55.

26. Brage $S$, Wedderkopp $N$, Ekelund $U$, et al. Objectively measured physical activity correlates with indices of insulin resistance in Danish children. The European Youth Heart Study (EYHS). Int J Obes Relat Metab Disord 2004; 28:1503-8.

27. Brage $S$, Wedderkopp $N$, Ekelund $U$, et al. Features of the metabolic syndrome are associated with objectively measured physical activity and fitness in Danish children: the European Youth Heart Study (EYHS). Diabetes Care 2004; 27:2141-8.

28. Brandao CM, Lombardi MT, Nishida SK, et al. Serum leptin concentration during puberty in healthy nonobese adolescents. Braz J Med Biol Res 2003; 36:1293-6. 
29. Braun B, Zimmermann MB, Kretchmer N, et al. Risk factors for diabetes and cardiovascular disease in young Australian aborigines. A 5-year followup study. Diabetes Care 1996; 19:472-9.

30. Braunschweig $\mathrm{CL}$, Gomez $\mathrm{S}$, Liang $\mathrm{H}$, et al. Obesity and risk factors for the metabolic syndrome among low-income, urban, African American schoolchildren: the rule rather than the exception? Am J Clin Nutr 2005; 81:970-5.

31. Brownson RC, Boehmer TK and Luke DA. Declining rates of physical activity in the United States: what are the contributors? Annual Review of Public Health 2005; 26:421-43.

32. Bruce RA, Kusumi $F$ and Hosmer D. Maximal oxygen intake and nomographic assessment of functional aerobic impairment in cardiovascular disease. Am Heart J 1973; 85:546-62.

33. Cammisotto PG and Bendayan M. Leptin secretion by white adipose tissue and gastric mucosa. Histol Histopathol 2007; 22:199-210.

34. Carlsson B, Ankarberg C, Rosberg S, et al. Serum leptin concentrations in relation to pubertal development. Arch Dis Child 1997; 77:396-400.

35. Choudhary AK, Donnelly LF, Racadio JM, et al. Diseases associated with childhood obesity. AJR Am J Roentgenol 2007; 188:1118-30.

36. Chu NF, Chang JB and Shieh SM. Plasma leptin, fatty acids, and tumor necrosis factor-receptor and insulin resistance in children. Obes Res 2003; 11:532-40.

37. Clarke HH. Academy approves physical fitness definition. Physical Fitness Newsletter 1979; 25:

38. Cohen B, Novick D and Rubinstein M. Modulation of insulin activities by leptin. Science 1996; 274:1185-8.

39. Colagiuri S, Sandbaek A, Carstensen B, et al. Comparability of venous and capillary glucose measurements in blood. Diab Med 2003; 20:953-6.

40. Cole TJ, Freeman JV and Preece MA. Body mass index reference curves for the UK, 1990. Arch Dis Child 1995; 73:25-9.

41. Cole TJ, Bellizzi MC, Flegal KM, et al. Establishing a standard definition for child overweight and obesity worldwide: international survey. Brit Med J 2000; 320:1240-3.

42. Cook $S$, Weitzman $M$, Auinger $P$, et al. Prevalence of a metabolic syndrome phenotype in adolescents: findings from the third National Health and Nutrition Examination Survey, 1988-1994. Arch Pediatr Adolesc Med 2003; 157:821-7.

43. Craig JG, van Holsbeeck $M$ and Zaltz I. The utility of MR in assessing Blount disease. Skeletal Radiol 2002; 31:208-13.

44. Cruz ML, Weigensberg MJ, Huang TT, et al. The metabolic syndrome in overweight Hispanic youth and the role of insulin sensitivity. J Clin Endocrinol Metab 2004; 89:108-13. 
45. Cumming GR, Everatt $\mathrm{D}$ and Hastman L. Bruce treadmill test in children: normal values in a clinic population. Am J Cardiol 1978; 41:69-75.

46. Curioni CC and Lourenco PM. Long-term weight loss after diet and exercise: a systematic review. Int J Obes (Lond) 2005; 29:1168-74.

47. Danadian K, Balasekaran G, Lewy V, et al. Insulin sensitivity in AfricanAmerican children with and without family history of type 2 diabetes. Diabetes Care 1999; 22:1325-9.

48. Dean H, Flett, B. Natural history of type 2 diabetes diagnosed in childhood: long term follow up in young adult years. Diabetes 2002; 51 (Suppl.2):

49. Demers J, Kane MP, Bakst G, et al. Accuracy of home blood glucose monitors using forearm blood samples: FreeStyle versus One Touch Ultra. Am J Health-Syst Phar 2003; 60:1130-5.

50. Deshmukh-Taskar P, Nicklas TA, Morales M, et al. Tracking of overweight status from childhood to young adulthood: the Bogalusa Heart Study. Eur J Clin Nutr 2006; 60:48-57.

51. DeStefano RA, Caprio S, Fahey JT, et al. Changes in body composition after a 12-wk aerobic exercise program in obese boys. Pediatr Diabetes 2000; 1:61-5.

52. Dietz WH. Overweight and precursors of type 2 diabetes mellitus in children and adolescents. J Pediatr 2001; 138:453-4.

53. DiMartino-Nardi J. Pre- and postpuberal findings in premature adrenarche. J Pediatr Endocrinol Metab 2000; 13 Suppl 5:1265-9.

54. Dollman J, Norton $\mathrm{K}$ and Norton L. Evidence for secular trends in children's physical activity behaviour. Br J Sports Med 2005; 39:892-7; discussion 897.

55. Duncan GE. Prevalence of diabetes and impaired fasting glucose levels among US adolescents: National Health and Nutrition Examination Survey, 1999-2002. Arch Ped Adol Med 2006; 160:523-8.

56. Ebbeling CB, Pawlak DB and Ludwig DS. Childhood obesity: public-health crisis, common sense cure. Lancet 2002; 360:473-82.

57. Ehtisham S, Barrett, T.G., Shaw, N.J. Type 2 diabetes mellitus in UK children - an emerging problem. Diabetic Medicine 2000; 17:867-871.

58. Eisenmann JC. Secular trends in variables associated with the metabolic syndrome of North American children and adolescents: a review and synthesis. Am J Hum Biol 2003; 15:786-94.

59. Eisenmann JC. Physical activity and cardiovascular disease risk factors in children and adolescents: an overview. Canadian Journal of Cardiology 2004; 20:295-301.

60. Eisenmann JC, Katzmarzyk PT, Perusse L, et al. Aerobic fitness, body mass index, and CVD risk factors among adolescents: the Quebec family study. Int J Obes (Lond) 2005; 29:1077-83. 
61. Ekeland E, Halland B, Refsnes KA, et al. [Are children and adolescents less physically active today than in the past?]. Tidsskr Nor Laegeforen 1999; 119:2358-62.

62. Eliakim A, Burke GS and Cooper DM. Fitness, fatness, and the effect of training assessed by magnetic resonance imaging and skinfold-thickness measurements in healthy adolescent females. American Journal of Clinical Nutrition 1997; 66:223-31.

63. Eliakim A, Kaven G, Berger I, et al. The effect of a combined intervention on body mass index and fitness in obese children and adolescents - a clinical experience. Eur J Pediatr 2002; 161:449-54.

64. Evans JM, Newton RW, Ruta DA, et al. Socio-economic status, obesity and prevalence of Type 1 and Type 2 diabetes mellitus. Diab Med 2000; 17:478-80.

65. Fagot-Campagna A, Burrows NR and Williamson DF. The public health epidemiology of type 2 diabetes in children and adolescents: a case study of American Indian adolescents in the Southwestern United States. Clin Chim Acta 1999; 286:81-95.

66. Fagot-Campagna A. Emergence of type 2 diabetes mellitus in children: epidemiological evidence. J Pediatr Endocrinol Metab 2000; 13 Suppl 6:1395-402.

67. Fagot-Campagna A, Pettitt DJ, Engelgau MM, et al. Type 2 diabetes among North American children and adolescents: an epidemiologic review and a public health perspective. J Pediatr 2000; 136:664-72.

68. Felson DT and Chaisson CE. Understanding the relationship between body weight and osteoarthritis. Baillieres Clin Rheumatol 1997; 11:671-81.

69. Ferreira I, Twisk JW, van Mechelen W, et al. Development of fatness, fitness, and lifestyle from adolescence to the age of 36 years: determinants of the metabolic syndrome in young adults: the amsterdam growth and health longitudinal study. Arch Intern Med 2005; 165:42-8.

70. Fiallo-Scharer R, Chase HP, Beck RW, et al. A multicenter study of the accuracy of the One Touch Ultra home glucose meter in children with type 1 diabetes. Diab Tech Ther 2003; 5:933-41.

71. Freedman DS, Ogden CL, Berenson GS, et al. Body mass index and body fatness in childhood. Current Opinion in Clinical Nutrition \& Metabolic Care 2005; 8:618-23.

72. Freedman DS, Wang J, Maynard LM, et al. Relation of BMI to fat and fatfree mass among children and adolescents. International Journal of Obesity (London) 2005; 29:1-8.

73. Freitas D, Maia J, Beunen G, et al. Socio-economic status, growth, physical activity and fitness: the Madeira Growth Study. Ann Hum Biol 2007; 34:107-22. 
74. Genuth S, Alberti KG, Bennett $P$, et al. Follow-up report on the diagnosis of diabetes mellitus. Diab Care 2003; 26:3160-7.

75. Gerver WJM, Bruin, de, R. Paediatric morphometrics. 2001, Maastricht: Datawyse / Universitaire Pers Maastricht.

76. Godina E, Khomyakova I, Purundzhan A, et al. Effect of physical training on body composition in Moscow adolescents. J Physiol Anthropol 2007; 26:229-34.

77. Golley RK, Magarey AM, Steinbeck KS, et al. Comparison of metabolic syndrome prevalence using six different definitions in overweight prepubertal children enrolled in a weight management study. Int J Obes (Lond) 2006; 30:853-60.

78. Goran M, Fields DA, Hunter GR, et al. Total body fat does not influence maximal aerobic capacity. Int J Obes Relat Metab Disord 2000; 24:841-8.

79. Goran MI. Metabolic precursors and effects of obesity in children: a decade of progress, 1990-1999. American Journal of Clinical Nutrition 2000; 73:158-171.

80. Goran MI, Bergman RN and Gower BA. Influence of total vs. visceral fat on insulin action and secretion in African American and white children. Obes Res 2001; 9:423-31.

81. Goran $\mathrm{MI}$ and Gower BA. Longitudinal study on pubertal insulin resistance. Diabetes 2001; 50:2444-50.

82. Goran MI, Coronges K, Bergman RN, et al. Influence of family history of type 2 diabetes on insulin sensitivity in prepubertal children. J Clin Endocrinol Metab 2003; 88:192-5.

83. Grinstein G, Muzumdar R, Aponte L, et al. Presentation and 5-year followup of type 2 diabetes mellitus in African-American and Caribbean-Hispanic adolescents. Horm Res 2003; 60:121-6.

84. Gulmans VA, de Meer K, Binkhorst RA, et al. Reference values for maximum work capacity in relation to body composition in healthy Dutch children. Eur Respir J 1997; 10:94-7.

85. Gungor N, Thompson T, Sutton-Tyrrell K, et al. Early signs of cardiovascular disease in youth with obesity and type 2 diabetes. Diabetes Care 2005; 28:1219-21.

86. Gutin B, Yin Z, Humphries MC, et al. Relations of moderate and vigorous physical activity to fitness and fatness in adolescents. Am J Clin Nutr 2005; 81:746-50.

87. Haines L, Wan KC, Lynn R, et al. Rising incidence of type 2 diabetes in children in the U.K. Diabetes Care 2007; 30:1097-101.

88. Hallal PC, Victora CG, Azevedo MR, et al. Adolescent physical activity and health: a systematic review. Sports Med 2006; 36:1019-30.

89. Haslam D. Obesity: a medical history. Obes Rev 2007; 8 Suppl 1:31-6. 
90. Hattori K, Tatsumi, N., Tanaka, S. Total body fat does not influence maximal aerobic capacity. American Journal of Human Biology 1997; 9:573-8.

91. Hirasing RA, Fredriks AM, van Buuren S, et al. [Increased prevalence of overweight and obesity in Dutch children, and the detection of overweight and obesity using international criteria and new reference diagrams]. Ned Tijdschr Gen 2001; 145:1303-8.

92. Hirschler V, Aranda C, Calcagno Mde L, et al. Can waist circumference identify children with the metabolic syndrome? Arch Pediatr Adolesc Med 2005; 159:740-4.

93. Huang KC, Lue BH, Yen RF, et al. Plasma adiponectin levels and metabolic factors in nondiabetic adolescents. Obes Res 2004; 12:119-24.

94. Huang TT, Ball GD and Franks PW. Metabolic syndrome in youth: current issues and challenges. Appl Physiol Nutr Metab 2007; 32:13-22.

95. Hung YJ, Chu NF, Wang SC, et al. Correlation of plasma leptin and adiponectin with insulin sensitivity and beta-cell function in children - the Taipei Children Heart Study. Int J Clin Pract 2006; 60:1582-7.

96. Hurk vd, K., Dommelen v, P., Wilde d, J.A., et al. [Overweight and obesity prevalence in 4-15 year old youth in the period 2002-2004], ed. TNO. 2006, Leiden.

97. Hussain A, Claussen B, Ramachandran A, et al. Prevention of type 2 diabetes: a review. Diabetes Res Clin Pract 2007; 76:317-26.

98. Imperatore G, Cheng YJ, Williams DE, et al. Physical activity, cardiovascular fitness, and insulin sensitivity among U.S. adolescents: the National Health and Nutrition Examination Survey, 1999-2002. Diabetes Care 2006; 29:1567-72.

99. Invitti C, Maffeis C, Gilardini L, et al. Metabolic syndrome in obese Caucasian children: prevalence using WHO-derived criteria and association with nontraditional cardiovascular risk factors. Int J Obes (Lond) 2006; 30:627-33.

100. Irving HM, Adlaf EM, Allison KR, et al. Trends in vigorous physical activity participation among Ontario adolescents, 1997-2001. Canadian Journal of Public Health 2003; 94:272-4.

101. Jolliffe CJ and Janssen I. Development of age-specific adolescent metabolic syndrome criteria that are linked to the Adult Treatment Panel III and International Diabetes Federation criteria. J Am Coll Cardiol 2007; 49:8918.

102. Kadiki OA, Reddy MR and Marzouk AA. Incidence of insulin-dependent diabetes (IDDM) and non-insulin-dependent diabetes (NIDDM) (0-34 years at onset) in Benghazi, Libya. Diabetes Res Clin Pract 1996; 32:165-73. 
103. Kasa-Vubu JZ, Lee CC, Rosenthal A, et al. Cardiovascular fitness and exercise as determinants of insulin resistance in postpubertal adolescent females. J Clin Endocrinol Metab 2005; 90:849-54.

104. Katzmarzyk PT, Leon AS, Wilmore $\mathrm{JH}$, et al. Targeting the metabolic syndrome with exercise: evidence from the HERITAGE Family Study. Med Sci Sports Exerc 2003; 35:1703-9.

105. Kawahara R, Amemiya T, Yoshino M, et al. Dropout of young non-insulindependent diabetics from diabetic care. Diabetes Res Clin Pract 1994; 24:181-5.

106. Khunti K, Stone MA, Bankart J, et al. Physical activity and sedentary behaviours of South Asian and white European children in inner city secondary schools in the UK. Fam Pract 2007; 24:237-44.

107. Kiess W, Reich A, Muller G, et al. Obesity in childhood and adolescence: clinical diagnosis and management. J Pediatr Endocrinol Metab 2001; 14 Suppl 6:1431-40.

108. Kiess W, Bottner, A., Raile, K., Kapellen, T., Muller, G., Galler, A., Paschke, Wabitsch, M. Type 2 diabetes mellitus in children and adolescents: a review from a european perspective. Hormone Research 2003; 59:77-84.

109. Kim CS, Park JS, Park J, et al. The relation between birth weight and insulin resistance in Korean adolescents. Yonsei Med J 2006; 47:85-92.

110. Kirk S, Scott BJ and Daniels SR. Pediatric obesity epidemic: treatment options. J Am Diet Assoc 2005; 105:S44-51.

111. Klein DJ, Aronson Friedman L, Harlan WR, et al. Obesity and the development of insulin resistance and impaired fasting glucose in black and white adolescent girls: a longitudinal study. Diabetes Care 2004; 27:378-83.

112. Klijn $\mathrm{PH}$, van der Baan-Slootweg $\mathrm{OH}$ and van Stel HF. Aerobic exercise in adolescents with obesity: preliminary evaluation of a modular training program and the modified shuttle test. BMC Pediatr 2007; 7:19.

113. Kosti RI and Panagiotakos DB. The epidemic of obesity in children and adolescents in the world. Cent Eur J Public Health 2006; 14:151-9.

114. Ku CY, Gower BA, Hunter GR, et al. Racial differences in insulin secretion and sensitivity in prepubertal children: role of physical fitness and physical activity. Obes Res 2000; 8:506-15.

115. LaMonte MJ, Barlow CE, Jurca $\mathrm{R}$, et al. Cardiorespiratory fitness is inversely associated with the incidence of metabolic syndrome: a prospective study of men and women. Circulation 2005; 112:505-12.

116. Lee S, Bacha F, Gungor N, et al. Waist circumference is an independent predictor of insulin resistance in black and white youths. J Pediatr 2006; 148:188-94. 
117. Leger LA, Mercier D, Gadoury C, et al. The multistage 20 meter shuttle run test for aerobic fitness. J Sports Sci 1988; 6:93-101.

118. Levitt Katz LE, Swami S, Abraham M, et al. Neuropsychiatric disorders at the presentation of type 2 diabetes mellitus in children. Pediatr Diabetes 2005; 6:84-9.

119. Libman I and Arslanian S. Type 2 diabetes in childhood: the American perspective. Horm Res 2003; 59 Suppl 1:69-76.

120. Likitmaskul S, Kiattisathavee $\mathrm{P}$, Chaichanwatanakul K, et al. Increasing prevalence of type 2 diabetes mellitus in Thai children and adolescents associated with increasing prevalence of obesity. J Pediatr Endocrinol Metab 2003; 16:71-7.

121. Lorente FO, Souville M, Griffet J, et al. Participation in sports and alcohol consumption among French adolescents. Addict Behav 2004; 29:941-6.

122. Macaluso CJ, Bauer UE, Deeb LC, et al. Type 2 diabetes mellitus among Florida children and adolescents, 1994 through 1998. Public Health Rep 2002; 117:373-9.

123. Matthews DR, Hosker JP, Rudenski AS, et al. Homeostasis model assessment: insulin resistance and beta-cell function from fasting plasma glucose and insulin concentrations in man. Diabetologia 1985; 28:412-9.

124. McArdle WD, Katch, F.I., Katch, V.L. Exercise physiology. Energy, nutrition, and human performance. 4th Edition ed. 1996, Baltimore: Williams \& Wilkins.

125. McCarthy HD. Body fat measurements in children as predictors for the metabolic syndrome: focus on waist circumference. Proc Nutr Soc 2006; 65:385-92.

126. McMurray RG, Harrell JS, Bangdiwala SI, et al. Tracking of physical activity and aerobic power from childhood through adolescence. Med Sci Sports Exerc 2003; 35:1914-22.

127. Montani JP, Viecelli AK, Prevot A, et al. Weight cycling during growth and beyond as a risk factor for later cardiovascular diseases: the 'repeated overshoot' theory. Int J Obes (Lond) 2006; 30 Suppl 4:S58-66.

128. Monzavi R, Dreimane D, Geffner ME, et al. Improvement in risk factors for metabolic syndrome and insulin resistance in overweight youth who are treated with lifestyle intervention. Pediatrics 2006; 117:e1111-8.

129. Monzillo LU and Hamdy O. Evaluation of insulin sensitivity in clinical practice and in research settings. Nutr Rev 2003; 61:397-412.

130. Moreno LA, Pineda I, Rodriguez G, et al. Waist circumference for the screening of the metabolic syndrome in children. Acta Paediatr 2002; 91:1307-12.

131. Mota J, Guerra S, Leandro C, et al. Association of maturation, sex, and body fat in cardiorespiratory fitness. American Journal of Human Biology 2002; 14:707-12. 
132. Mul D, Fredriks AM, van Buuren S, et al. Pubertal development in The Netherlands 1965-1997. Pediatr Res 2001; 50:479-86.

133. Mulkens S, Fleuren, D., Nederkoorn, C., Meijers, J. [Realtfit:a multidisciplinary group treatment for overweigth youth]. Gedragstherapie 2007; 40:27-48.

134. Nassis GP, Papantakou K, Skenderi K, et al. Aerobic exercise training improves insulin sensitivity without changes in body weight, body fat, adiponectin, and inflammatory markers in overweight and obese girls. Metabolism 2005; 54:1472-9.

135. National-Task-Force-on-the-Prevention-and-Treatment-of-Obesity. Weight cycling. in Jama. 1994.

136. Nikolic Z and Ilic N. Maximal oxygen uptake in trained and untrained 15year-old boys. British Journal of Sports Medicine 1992; 26:36-8.

137. Ogden $\mathrm{CL}$, Carroll MD and Flegal KM. Epidemiologic trends in overweight and obesity. Endocrinol Metab Clin North Am 2003; 32:741-60, vii.

138. Olds $\mathrm{T}$, Tomkinson $\mathrm{G}$, Leger $\mathrm{L}$, et al. Worldwide variation in the performance of children and adolescents: an analysis of 109 studies of the 20-m shuttle run test in 37 countries. J Sports Sci 2006; 24:1025-38.

139. Olds TS, Ridley K and Tomkinson GR. Declines in aerobic fitness: are they only due to increasing fatness? Med Sport Sci 2007; 50:226-40.

140. Olshansky SJ, Passaro DJ, Hershow RC, et al. A potential decline in life expectancy in the United States in the 21st century. N Engl J Med 2005; 352:1138-45.

141. Organisation $\mathrm{WH}$, Obesity: preventing and managing the global epidemic, in Report of a WHO consultation, Geneva, 3-5 June1997. 1998: Geneva.

142. Owen KR and McCarthy Ml. Genetics of type 2 diabetes. Curr Opin Genet Dev 2007;

143. Pate RR, Pratt M, Blair SN, et al. Physical activity and public health. A recommendation from the Centers for Disease Control and Prevention and the American College of Sports Medicine. Jama 1995; 273:402-7.

144. Perichart-Perera O, Balas-Nakash M, Schiffman-Selechnik E, et al. Obesity increases metabolic syndrome risk factors in school-aged children from an urban school in Mexico city. J Am Diet Assoc 2007; 107:81-91.

145. Pinhas-Hamiel O, Dolan LM, Daniels SR, et al. Increased incidence of noninsulin-dependent diabetes mellitus among adolescents. J Pediatr 1996; 128:608-15.

146. Pinhas-Hamiel $\mathrm{O}$ and Zeitler P. The global spread of type 2 diabetes mellitus in children and adolescents. J Pediatr 2005; 146:693-700.

147. Power C, Lake JK and Cole TJ. Body mass index and height from childhood to adulthood in the 1958 British born cohort. Am J Clin Nutr 1997; 66:1094-101. 
148. Proctor $\mathrm{MH}$, Moore LL, Gao D, et al. Television viewing and change in body fat from preschool to early adolescence: The Framingham Children's Study. Int J Obes Relat Metab Disord 2003; 27:827-33.

149. Ramachandran A, Snehalatha C, Satyavani K, et al. Type 2 diabetes in Asian-Indian urban children. Diabetes Care 2003; 26:1022-5.

150. Rami B, Schober E, Nachbauer E, et al. Type 2 diabetes mellitus is rare but not absent in children under 15 years of age in Austria. Eur J Pediatr 2003; 162:850-2.

151. Reinehr T, Brylak K, Alexy U, et al. Predictors to success in outpatient training in obese children and adolescents. Int J Obes Relat Metab Disord 2003; 27:1087-92.

152. Renders CM, Delemarre-van de Waal HA, Dekker JM, et al. [Insulin resistance and type 2 diabetes in overweight children]. Ned Tijdschr Geneeskd 2003; 147:2060-3.

153. Rhodes SK, Shimoda KC, Waid LR, et al. Neurocognitive deficits in morbidly obese children with obstructive sleep apnea. J Pediatr 1995; 127:741-4.

154. Richards RJ, Svec F, Bao W, et al. Steroid hormones during puberty: racial (black-white) differences in androstenedione and estradiol--the Bogalusa Heart Study. J Clin Endocrinol Metab 1992; 75:624-31.

155. Rizzo NS, Ruiz JR, Hurtig-Wennlof A, et al. Relationship of physical activity, fitness, and fatness with clustered metabolic risk in children and adolescents: the European youth heart study. J Pediatr 2007; 150:388-94.

156. Rodriguez G, Moreno LA, Blay MG, et al. Body composition in adolescents: measurements and metabolic aspects. Int J Obes Relat Metab Disord 2004; 28 Suppl 3:S54-8.

157. Roemmich JN, Clark PA, Berr SS, et al. Gender differences in leptin levels during puberty are related to the subcutaneous fat depot and sex steroids. Am J Physiol 1998; 275:E543-51.

158. Rotteveel J, Belksma EJ, Renders CM, et al. Type 2 diabetes in children in the Netherlands: the need for diagnostic protocols. Eur J Endocrinol 2007; 157:175-80.

159. Rowland TW. Evolution of maximal oxygen uptake in children. Med Sport Sci 2007; 50:200-9.

160. Ruige JB, Dekker JM, Nijpels G, et al. Hyperproinsulinaemia in impaired glucose tolerance is associated with a delayed insulin response to glucose. Diabetologia 1999; 42:177-80.

161. Rump P, Verstappen F, Gerver WJ, et al. Body composition and cardiorespiratory fitness indicators in prepubescent boys and girls. Internaltional Journal of Sports Medicine 2002; 23:50-4. 
162. Rump P, Mensink, R.P., Kester, A.D.M., Hornstra, G. Essential fatty acid composition of plasma phospholipids and birth weight: a study in term neonates. American Journal of Clinical Nutrition 2001; 73:797-806.

163. Rump P, Popp-Snijders, C., Heine, R.J., Hornstra, G. Components of the insulin resistance syndrome in seven-year-old children: relations with birth weights and the polyunsaturated fatty acid content of umbilical cord plasma phospholipids. Diabetologia 2002; 45:349-355.

164. Saland JM. Update on the metabolic syndrome in children. Curr Opin Pediatr 2007; 19:183-91.

165. Schober E, Holl RW, Grabert $M$, et al. Diabetes mellitus type 2 in childhood and adolescence in Germany and parts of Austria. Eur J Pediatr 2005; 164:705-7.

166. Schuit AJ, Leest, van, L.A.T.M., [How many people are physically active enough?], in Volksgezondheid Toekomst Verkenning, RIVM, Editor. 2005: Bilthoven.

167. Shaibi GQ, Cruz ML, Ball GD, et al. Cardiovascular fitness and the metabolic syndrome in overweight latino youths. Med Sci Sports Exerc 2005; 37:922-8.

168. Shaibi GQ, Ball GD, Cruz ML, et al. Cardiovascular fitness and physical activity in children with and without impaired glucose tolerance. Int J Obes (Lond) 2006; 30:45-9.

169. Shaibi GQ, Cruz ML, Weigensberg MJ, et al. Adiponectin independently predicts metabolic syndrome in overweight Latino youth. J Clin Endocrinol Metab 2007; 92:1809-13.

170. Shalitin S and Phillip M. Role of obesity and leptin in the pubertal process and pubertal growth--a review. Int J Obes Relat Metab Disord 2003; 27:869-74.

171. Sinacore DR and Gulve EA. The role of skeletal muscle in glucose transport, glucose homeostasis, and insulin resistance: implications for physical therapy. Phys Ther 1993; 73:878-91.

172. Sinha R, Fisch G, Teague B, et al. Prevalence of impaired glucose tolerance among children and adolescents with marked obesity. N Engl J Med 2002; 346:802-10.

173. Slinger JD, van Breda $\mathrm{E}$, Keizer $\mathrm{H}$, et al. Insulin resistance, physical fitness, body composition and leptin concentration in 7-8 year-old children. J Sci Med Sport 2007; In press.

174. Slinger JD, Verstappen, F.T., Van Breda, E., Kuipers, H. The effect of body build and BMI on aerobic test performance in school children (10-15 years). J Sports Sci \& Med 2006; 5:699-706.

175. Snethen JA, Broome ME and Cashin SE. Effective weight loss for overweight children: a meta-analysis of intervention studies. J Pediatr Nurs 2006; 21:45-56. 
176. Someshwar J, Someshwar S and Perkins KC. The obese adolescent. Pediatr Ann 2006; 35:180-6.

177. Sorof JM, Poffenbarger T, Franco K, et al. Isolated systolic hypertension, obesity, and hyperkinetic hemodynamic states in children. J Pediatr 2002; 140:660-6.

178. Stefan N, Bunt JC, Salbe AD, et al. Plasma adiponectin concentrations in children: relationships with obesity and insulinemia. J Clin Endocrinol Metab 2002; 87:4652-6.

179. Steinberger J, Steffen L, Jacobs DR, Jr., et al. Relation of leptin to insulin resistance syndrome in children. Obes Res 2003; 11:1124-30.

180. Strauss RS and Pollack HA. Epidemic increase in childhood overweight, 1986-1998. Jama 2001; 286:2845-8.

181. Sturm R. Childhood obesity - what we can learn from existing data on societal trends, part 2. Prev Chronic Dis 2005; 2:A20.

182. Sturm R. Childhood obesity - what we can learn from existing data on societal trends, part 1. Prev Chronic Dis 2005; 2:A12.

183. Sugerman HJ, Sugerman EL, DeMaria EJ, et al. Bariatric surgery for severely obese adolescents. J Gastrointest Surg 2003; 7:102-7; discussion 107-8.

184. Sullivan S, Cloninger CR, Przybeck TR, et al. Personality characteristics in obesity and relationship with successful weight loss. Int J Obes (Lond) 2007; 31:669-74.

185. Summerbell CD, Ashton V, Campbell KJ, et al. Interventions for treating obesity in children. Cochrane Database Syst Rev 2003; CD001872.

186. Svensson M, Sundkvist G, Arnqvist HJ, et al. Signs of nephropathy may occur early in young adults with diabetes despite modern diabetes management: results from the nationwide population-based Diabetes Incidence Study in Sweden (DISS). Diabetes Care 2003; 26:2903-9.

187. Swartz AM, Strath, S.J., Bassett, D.R., Moore, J.B., Redwine, B.A., Groer, M.R.N., Thompson, D.L. Increasing daily walking improves glucose tolerance in overweight women. Preventive Medicine 2003; 37:356-362.

188. Sygusch R. [Youth sport-youth health. An overview of the current state of research]. Bundesgesundheitsblatt Gesundheitsforschung Gesundheitsschutz 2005; 48:863-72.

189. Teixeira PJ, Going SB, Houtkooper LB, et al. Pretreatment predictors of attrition and successful weight management in women. Int J Obes Relat Metab Disord 2004; 28:1124-33.

190. Ten S and Maclaren N. Insulin resistance syndrome in children. J Clin Endo Metab 2004; 89:2526-39.

191. Tomkinson GR, Leger LA, Olds TS, et al. Secular trends in the performance of children and adolescents (1980-2000): an analysis of 55 studies of the 20m shuttle run test in 11 countries. Sports Med 2003; 33:285-300. 
192. Tomkinson GR, Olds TS, Kang SJ, et al. Secular trends in the aerobic fitness test performance and body mass index of korean children and adolescents (1968 - 2000). Int J Sports Med 2007; 28:314-20.

193. Ulmer H, Kelleher C, Diem G, et al. Long-term tracking of cardiovascular risk factors among men and women in a large population-based health system: the Vorarlberg Health Monitoring \& Promotion Programme. Eur Heart J 2003; 24:1004-13.

194. Urakami T, Kubota S, Nitadori Y, et al. Annual incidence and clinical characteristics of type 2 diabetes in children as detected by urine glucose screening in the Tokyo metropolitan area. Diabetes Care 2005; 28:187681.

195. van den Hurk K, van Dommelen P, van Buuren S, et al. Prevalence of overweight and obesity in the Netherlands in 2003, compared to 1980 and 1997. Arch Dis Child 2007;

196. Van Etten LM, Verstappen FT and Westerterp KR. Effect of body build on weight-training-induced adaptations in body composition and muscular strength. Medicine and Science in Sports and Exercise 1994; 26:515-21.

197. van Mechelen W, Hlobil $\mathrm{H}$ and Kemper HC. Validation of two running tests as estimates of maximal aerobic power in children. Eur J Appl Physiol Occup Physiol 1986; 55:503-6.

198. van Mechelen W, Lier, van W.H., Hlobil, H., Crolla, I., Kemper, H.C.G. Eurofit: handleiding met referentieschalen voor 12- tot en met 16-jarige jongens en meisjes in Nederland. 1991, Haarlem: De Vrieseborch.

199. Vanltallie TB, Yang MU, Heymsfield SB, et al. Height-normalized indices of the body's fat-free mass and fat mass: potentially useful indicators of nutritional status. American Journal of Clinical Nutrition 1990; 52:953-9.

200. Vasudev S, Mohan A, Mohan D, et al. Validation of body fat measurement by skinfolds and two bioelectric impedance methods with DEXA-the Chennai Urban Rural Epidemiology Study [CURES-3]. Journal of the Association of Physicians of India 2004; 52:877-81.

201. Visscher TLS, [How many people are overweight?], in Volksgezondheids Toekomst Verkenning, Nationaal Kompas Volksgezondheid., RIVM, Editor. 2006, www.nationaalkompas.nl: Bilthoven.

202. Voedingscentrum, [Health risks of being overweight are unknown in de Dutch population], www.voedingscentrum.nl, Editor. 2004.

203. Voedingscentrum, [Overweight in numbers], www.voedingscentrum.nl, Editor. 2007.

204. Vos C, Overheid onderschat probleem vetzucht, in Volkskrant. 2007: Amsterdam. p. $1 \& 7$.

205. Wang $\mathrm{Y}, \mathrm{Ge} \mathrm{K}$ and Popkin BM. Tracking of body mass index from childhood to adolescence: a 6-y follow-up study in China. Am J Clin Nutr 2000; 72:1018-24. 
206. Watanabe K, Nakadomo F and Maeda K. Relationship between body composition and cardiorespiratory fitness in Japanese junior high school boys and girls. Annals of Physiological Anthropology 1994; 13:167-74.

207. Weinzimer SA, Beck RW, Chase HP, et al. Accuracy of newer-generation home blood glucose meters in a Diabetes Research in Children Network (DirecNet) inpatient exercise study. Diabetes Tech Therapeut 2005; 7:67580; discussion 681-3.

208. Weiss R, Dziura J, Burgert TS, et al. Obesity and the metabolic syndrome in children and adolescents. N Engl J Med 2004; 350:2362-74.

209. Weiss R and Caprio S. The metabolic consequences of childhood obesity. Best Pract Res Clin Endocrinol Metab 2005; 19:405-19.

210. Wells JC. A critique of the expression of paediatric body composition data. Archives of Disease in Childhood 2001; 85:67-72.

211. Wells JC and Cole TJ. Adjustment of fat-free mass and fat mass for height in children aged $8 \mathrm{y}$. International Journal of Obesity Related Metabolic Disorders 2002; 26:947-52.

212. Wendel-Vos GC, Schuit AJ, Saris WH, et al. Reproducibility and relative validity of the short questionnaire to assess health-enhancing physical activity. J Clin Epidemiol 2003; 56:1163-9.

213. Westerstahl M, Barnekow-Bergkvist M, Hedberg G, et al. Secular trends in body dimensions and physical fitness among adolescents in Sweden from 1974 to 1995. Scandinavian Journal of Medicine and Science in Sports 2003; 13:128-37.

214. Weststrate JA and Deurenberg P. Body composition in children: proposal for a method for calculating body fat percentage from total body density or skinfold-thickness measurements. Am J Clin Nutr 1989; 50:1104-15.

215. WHO, Obesity: preventing and managing the global epidemic. Report of a WHO consultation, in World Health Organ Tech Rep Ser. 2000. p. i-xii, 1253.

216. WHO, Obesity and overweight, in Fact Sheet, www.who.int, Editor. 2006.

217. WHO, Diabetes, in Fact sheet, www.who.int, Editor. 2006.

218. Williams CL, Campanaro LA, Squillace M, et al. Management of childhood obesity in pediatric practice. Ann N Y Acad Sci 1997; 817:225-40.

219. Yamauchi T, Kim SN, Lu Z, et al. Age and gender differences in the physical activity patterns of urban schoolchildren in Korea and China. J Physiol Anthropol 2007; 26:101-7.

220. Yoshida Y, Hagura R, Hara Y, et al. Risk factors for the development of diabetic retinopathy in Japanese type 2 diabetic patients. Diabetes Res Clin Pract 2001; 51:195-203.

221. Yoshinaga M, Tanaka S, Shimago A, et al. Metabolic syndrome in overweight and obese Japanese children. Obes Res 2005; 13:1135-40. 
222. Young TK, Martens PJ, Taback SP, et al. Type 2 diabetes mellitus in children: prenatal and early infancy risk factors among native canadians. Arch Pediatr Adolesc Med 2002; 156:651-5. 
SUMMARY 
The prevalence of overweight and obesity has increased worldwide both in adults and in youth over the last decades, and this trend is expected to continue in the future. One of the most prominent health consequences of overweight is the development of insulin resistance, which may develop into full-blown type 2 Diabetes Mellitus (type 2 DM). Nowadays, the increased prevalence of overweight and insulin resistance in youth is suggested to be, at least partly, caused by a decrease in physical activity and/or fitness level. To be able to develop effective prevention programs for this group in the Netherlands, it is important to have in depth knowledge about physical fitness and its associations with body composition and insulin resistance in the Dutch youth population. Therefore, the general aim of the current thesis was to investigate possible associations between body composition (fat mass and fat free mass), aerobic fitness and insulin resistance in Dutch adolescents living in the province of Limburg.

For that purpose, in the second Chapter of the current thesis, possible associations between body composition, aerobic test performance and insulin resistance (measured by means of homeostatic model assessment for insulin resistance: HOMA-IR) were studied in a group of children who were on average 7 years old. The results showed that leptin (a hormone produced by fat tissue) appears to be an important factor associated with insulin resistance, whereas the influence of a positive family history on insulin resistance appeared, unexpectedly, only as a trend at this young age. Furthermore, we suggest that physical activity, and to a lesser extent physical fitness, may have an indirect protective role in the development of insulin resistance in young children by modulating body composition and leptin synthesis. Moreover, the importance of the indirect role of physical activity has also been mentioned in the same group at the age of 13 as described in Chapter 3. The difference in results between the two age groups was that at the age of 13 leptin concentration was not associated with insulin resistance.

Focusing on the development of relevant parameters in the group between the age of 7 and 13, the results showed that while the glucose concentration remained stable, HOMA-IR, insulin, and leptin concentrations increased over time. This suggests that the increase in insulin resistance was compensated by an increased insulin concentration. Additionally, a significant tracking was demonstrated for anthropometric parameters, HOMA-IR, insulin concentration and leptin concentration after adjustment for gender. This implies that the level of insulin resistance at the age of 13 can partly be predicted by its level at the age of 7 .

In Chapter 4 the prevalence of an elevated glucose concentration (which can be an early sign of insulin resistance) in the 12-16 year old population is presented. It is concluded that in a single measurement $15 \%$ of the Dutch 
adolescents showed elevated glucose levels, whereas about $3 \%$ of the population showed a confirmed (based on a second measurement) elevated glucose level. Based on the previously described consistent increase in body weight, we strongly think that the prevalence of impaired glucose concentration will continue to rise. Therefore, the prevalence of impaired glucose tolerance should regularly be monitored in order to initiate prevention activities, when appropriate. In the population studied in Chapter 4, the data suggest that the glucose concentration is related to age, educational level and BMI. In the population described in chapter 3 a positive family history of diabetes and an elevated fat mass index (fat mass/height ${ }^{2}$ ) have been identified as risk factors. This indicates that at the age studied extra attention should be given to the children who are at the pre-vocational education level, those with a positive family history and to those who have an above normal BMI or more specifically an elevated fat mass index.

Although body composition seems to be the most important link with insulin resistance, physical fitness has also been suggested to be, although indirectly, involved. Chapter 5 focused on the association between physical fitness and body build. Body build can be assessed by means of the fat mass index (fat mass/height ${ }^{2}$ ) and the fat free mass index (fat free mass/height ${ }^{2}$ ). Individuals with a high fat free mass index in relation to their fat mass index are considered to have a solid body build, whereas individuals with a relative low fat free mass are considered slender. In Chapter 5 we showed that in the age group of 11 to 15 years, aerobic test performance, as normalized to body weight, was superior in individuals with a solid body build over individuals with a slender body build. However, it has also been shown that the power output relative to fat free mass, as measured by a cycle ergometer test, was comparable over the three groups. This suggests that the muscle mass of adolescents with a more healthy body build (solid) are comparable with this mass in adolescents with a less healthy body build (slender) implying that the physical activity level between the groups may be comparable and that the differences in body build are mainly determined by genetic predisposition.

Comparing the current absolute aerobic test performance data with data from 15 years ago, in Chapter 6 we showed that aerobic performance capacity has not changed in Dutch adolescents during this period. However, current aerobic test performance normalized to body weight is lower than previous reference values for the Netherlands. Although such a decrease in fitness level normalized to body weight may be due to the increase in body fat in youth rather than deterioration in cardiovascular function, it may have other implications on health than the influence of extra body fat itself. Due to this suggested (indirect) association between 
physical fitness and several health risks, the level of physical fitness should regularly be evaluated. In these tests attention should be paid to standardization of fitness tests, because differences in currently used tests hinder proper comparison over time and comparison between countries.

With the ongoing rise in overweight and insulin resistance prevalence, several prevention programs have been launched aiming to prevent or reverse the associated upcoming health problems in youth. One of these programs is Realfit, which is a community based program for overweight adolescents as described in Chapter 7. The results of the study showed that Realfit had a positive effect on body weight, BMI, waist circumference and parameters related to the metabolic syndrome on the short-term (after 12 weeks of program). However, long-term results (20 weeks after the end of the program) showed that $\mathrm{BMI}$ and waist circumference remained at the post-program level, whereas body weight returned to baseline (pre-program) level. These findings emphasize the importance of long-term management, which often lacks in such programs because of organizational and/or financial reasons. In the group of successful participants (those who decreased their $\mathrm{BMI}$ ), a decrease in insulin resistance could be shown on the short-term indicating a potential preventive effect of community based intervention programs in overweight adolescents for the development of insulin resistance.

In Chapter 8 the most important results concerning body composition, metabolic derangements and physical fitness have been discussed. Implications for prevention and intervention are described and recommendations for practical use and for future research are listed. In conclusion, the data from the current thesis showed no direct association between physical fitness and insulin resistance in youth. However, it is likely that physical activity and good physical fitness may diminish the problems associated with overweight and insulin resistance in youth. With the current increase in prevalence of overweight and decrease in fitness level in adolescents a further increase in the prevalence of insulin resistance and type 2 DM in Dutch children and adolescents is anticipated with major social and economic consequences. It is, therefore, strongly recommended to focus, and develop, early preventive measures. 
SAMENVATTING 
De wereldwijde prevalentie van overgewicht en obesitas is zowel bij volwassenen als bij jongeren gestegen en het is de verwachting dat deze stijging zich in de toekomst zal voortzetten. Een van de belangrijkste gezondheidsrisico's van overgewicht is het ontwikkelen van insuline resistentie, wat een voorstadium van Diabetes Mellitus type 2 (type 2 DM) kan zijn. De gestegen prevalentie van overgewicht en insulineresistentie bij jongeren kan, op zijn minst gedeeltelijk, worden verklaard door een verminderd fysiek activiteitsniveau en een verminderde fitheid. Om effectieve preventieprogramma's voor deze doelgroep te kunnen ontwikkelen is het daarom belangrijk voldoende kennis te hebben van fysieke fitheid en de associaties met lichaamssamenstelling en insuline resistentie bij Nederlandse jongeren. Het algemene doel van de onderzoeken die zijn beschreven in dit proefschrift is dan ook het bestuderen van mogelijke relaties tussen lichaamssamenstelling (vet massa en vet vrije massa), aërobe capaciteit en insuline resistentie in Nederlandse jongeren uit de provincie Limburg.

In Hoofdstuk 2 van dit proefschrift worden mogelijke relaties tussen lichaamssamenstelling, aëroob vermogen en insuline resistentie (geschat d.m.v. homeostatische model meting voor insuline resistentie (HOMA-IR)) onderzocht in een groep kinderen van gemiddeld zeven jaar oud. De resultaten lieten zien dat leptine (een hormoon dat wordt geproduceerd door het vetweefsel) een belangrijke factor bleek te zijn, die is geassocieerd met insuline resistentie. De invloed van de familiaire achtergrond met betrekking tot diabetes bleek, tegen de verwachting in, geen significante relatie te vertonen met insuline resistentie op deze leeftijd. Verder hebben we in dit hoofdstuk gesuggereerd dat fysieke activiteit en in mindere mate fysieke fitheid door de invloed op lichaamssamenstelling en de aanmaak van leptine een indirecte beschermende rol speelt bij de ontwikkeling van insuline resistentie bij kinderen. Deze indirecte rol kwam ook naar voren wanneer dezelfde groep werd gemeten op 13 jarige leeftijd, zoals beschreven in Hoofdstuk 3. Het verschil tussen de resultaten op beide leeftijden is dat er op 13 jarige leeftijd geen significante associatie meer werd gevonden tussen leptine en insuline resistentie.

Wanneer we de ontwikkeling van de relevante parameters tussen het $7^{\mathrm{e}}$ en $13^{\mathrm{e}}$ levensjaar bekeken, bleek dat de glucose concentratie vergelijkbaar was, terwijl HOMA-IR en insuline en leptine concentraties stegen. Dit duidt er op dat gestegen insuline resistentie wordt gecompenseerd door verhoogde insuline concentraties. Daarnaast bleek dat na correctie voor geslacht de tracking (associatie tussen waarden van dezelfde parameter gemeten op verschillende momenten) van de anthropometrische variabelen, HOMA-IR, insuline concentratie en leptine concentratie, significant was tussen de twee meetmomenten. Dit impliceert dat het insuline resistentie 
niveau op 13 jarige leeftijd gedeeltelijk wordt voorspeld door het niveau op 7 jarige leeftijd.

In Hoofdstuk 4 wordt de prevalentie van een verhoogde glucose concentratie (wat een voorteken van insuline resistentie kan zijn) gepresenteerd voor 12 tot 16 jarigen. In een eenmalige meting werd bij $15 \%$ van de deelnemers een verhoogde glucose concentratie gevonden, terwijl bij ongeveer 3\% van de totale groep een bevestigde verhoging kon worden aangetoond bij een tweede meting. Gebaseerd op de eerder genoemde stijging in lichaamsgewicht in de doelgroep, kan worden verwacht dat de prevalentie van een verstoorde glucose concentratie in de toekomst ook verder zal stijgen. Daarom is het belangrijk om deze prevalentie regelmatig te meten om op het juiste moment preventieve activiteiten te starten. In de bestudeerde populatie tonen de data dat de gemeten glucose concentratie gerelateerd is aan leeftijd, opleidingsniveau en BMI. In de onderzoekspopulatie zoals beschreven in Hoofdstuk 3 werden een familiaire achtergrond van diabetes en een verhoogde vet massa index (vet massa/lengte ${ }^{2}$ ) geïdentificeerd als risicofactoren voor insuline resistentie. Dit suggereert dat in deze leeftijdscategorie extra aandacht moet worden besteed aan jongeren die het VMBO niveau volgen, jongeren met een positieve familiaire achtergrond en jongeren met een verhoogde BMI en meer specifiek een verhoogde vet massa index.

Hoewel lichaamssamenstelling de belangrijkste voorspeller van insuline resistentie blijkt te zijn, lijkt ook fysieke fitheid een indirecte rol te spelen. In Hoofdstuk 5 is de relatie tussen lichaamsbouw en fysieke fitheid beschreven. Lichaamsbouw kan o.a. worden gemeten door de vet massa index (vet massa/lengte ${ }^{2}$ ) en de vet vrije massa index (vet vrije massa/lengte ${ }^{2}$ ). De lichaamsbouw van personen met een hoge vet vrije massa index in verhouding tot de vet massa index kan 'solid' (gespierd) genoemd worden, terwijl de bouw van personen met een relatief lage vet vrije massa index 'slender' (weinig gespierd) genoemd wordt. In Hoofdstuk 5 hebben we laten zien dat in de categorie 11 tot 15 jarigen de aërobe capaciteit, genormaliseerd voor lichaamsgewicht, beter was voor de personen met een 'solid' lichaamsbouw. Wanneer we echter de capaciteit uitdrukken per kilogram vet vrije massa blijkt de capaciteit vergelijkbaar in de twee groepen. Dit suggereert dat de spiermassa van adolescenten met een gezonde of minder gezonde lichaamsbouw vergelijkbaar zijn, wat duidt op een vergelijkbaar activiteitsniveau. Wanneer we ervan uitgaan dat het activiteitsniveau vergelijkbaar is, lijken de verschillen in lichaamsbouw vooral gebaseerd te zijn op genetische aanleg.

Wanneer we de absolute aërobe capaciteit zoals gemeten in onze studies vergelijken met het niveau van vijftien jaar geleden, zoals omschreven in Hoofdstuk 6, blijkt dat deze absolute waarde niet is veranderd gedurende 
deze periode. Wanneer de aërobe capaciteit echter wordt genormaliseerd voor lichaamsgewicht blijkt de capaciteit te zijn gedaald. Deze daling kan waarschijnlijk meer worden toegeschreven aan een stijging van het lichaamsgewicht dan aan een daling van de aërobe capaciteit. Aangezien fitheid (indirect) gerelateerd lijkt te zijn aan verschillende gezondheidsrisico's, kan worden geadviseerd het fitheidniveau van de Nederlandse jeugd regelmatig te controleren. Bij het uitvoeren van deze testen moet overigens wel aandacht worden besteed aan de standaardisatie van de tests, aangezien verschillen in de gebruikte testen een vergelijking over de tijd of tussen landen bemoeilijkt.

Onder invloed van de toename van overgewicht en insuline resistentie, zijn verschillende programma's ontwikkeld die het voorkomen of terugdringen van de opkomende gezondheidsproblemen bij jongeren tot doel hebben. Een van deze programma's is Realfit, een programma dat is ontstaan vanuit de samenleving en zich richt op jongeren met overgewicht, zoals beschreven in Hoofdstuk 7. De resultaten laten zien dat Realfit op de korte termijn (na 12 weken programma) een positief effect had op lichaamsgewicht, BMI, middelomtrek en de parameters behorende bij het metabool syndroom. Op de lange termijn (20 weken na afloop van het programma) bleken BMI en middelomtrek ongeveer op hetzelfde niveau zijn gebleven als op de korte termijn, terwijl het lichaamsgewicht weer was gestegen tot het niveau bij aanvang. Deze resultaten benadrukken het belang van lange termijn begeleiding, terwijl juist deze begeleiding door organisatorische en/of financiële overwegingen in een programma vaak ontbreekt. In de groep succesvolle deelnemers (waarbij de BMI daalde tijdens het programma), daalde ook de insuline resistentie op de korte termijn. Dit duidt op een potentieel preventief effect van dergelijke programma's voor jongeren met overgewicht met betrekking tot het ontwikkelen van insuline resistentie.

In Hoofdstuk 8 worden de belangrijkste resultaten met betrekking tot lichaamssamenstelling, metabole problemen en fysieke fitheid bediscussieerd. Gevolgen voor preventie en interventie worden besproken en aanbevelingen voor de praktijk en voor verder onderzoek worden opgesomd. Concluderend kan worden gesteld dat de data gepresenteerd in dit proefschrift laten zien dat er geen directe relatie bestond tussen fysieke fitheid en insuline resistentie bij jongeren. Het mag echter worden verwacht dat fysieke activiteit en een goede fysieke fitheid de problemen die geassocieerd zijn met overgewicht bij jongeren zullen doen verminderen. Met de huidige toename in de prevalentie van overgewicht en de verminderde fitheid van adolescenten kan een verdere toename in de prevalentie van insuline resistentie en type 2 DM in deze groep worden 
verwacht, met de nodige sociale en economische gevolgen. Daarom wordt geadviseerd meer aandacht te besteden aan preventieve activiteiten. 
APPENDIX

Dankwoord

Curriculum Vitae

Publications

List of abbreviations 


\section{DANKWOORD}

Hoe sterk is de eenzame fietser

die kromgebogen over zijn stuur

tegen de wind

zich zelf een weg baant...

Als de fietser het echt wil zal 'ie zeker op de bestemming aankomen, maar met mensen die hem af en toe uit de wind houden, wijzen op de mooie dingen naast de weg of zelfs even aanmoedigen zal de rit een stuk plezieriger worden. Dat zelfde geldt voor mijn promotietraject, vandaar dit dankwoord.

De allerbelangrijkste groep mensen die ik wil bedanken zijn alle deelnemers aan dit onderzoek. Zonder jullie was er helemaal geen route geweest om te fietsen. Ik vind het stoer hoe jullie je angsten hebben overwonnen en de testjes hebben doorstaan!

Harm en Eric wil ik bedanken voor de begeleiding tijdens dit traject. Harm, jouw nuchtere kijk op onderzoek en het schrijfproces hebben mij vaak weer de juiste richting op gestuurd. Eric, een speciaal bedankje voor alle kerstbomen die je me hebt gestuurd de afgelopen jaren!

Beste leden van de beoordelingscommissie bedankt voor het kritisch lezen van het manuscript. Rob, ik wil jou en je collega's daarnaast ook bedanken voor het organiseren van de epi master. Alle co-auteurs wil ik bedanken voor het kritisch mee organiseren, schrijven en lezen van het manuscript.

Ik heb het geluk gehad tijdens dit project met veel externe organisaties te hebben kunnen samenwerken, die samen met mij de weg hebben aangelegd. De samenwerking met de Gemeente Maastricht, Groene kruis, Huis voor de Sport Limburg, Topfit, GGD, Sint Maartenscollege, VMBOWest en Trajectumcollege waren een waardevolle toevoeging voor het onderzoek, maar vooral het samenwerken met de mensen achter de organisaties maakte het een plezierige samenwerking. Hein, Frank, Jenny, Petra, Roel, Rob, Joris, Chantal, Hans, Harry, Jan; dank jullie wel!

Graag wil ik alle collega's van de afdeling Bewegingswetenschappen (Bart A, Bart P, Berber, Désirée, Ellen L, Ellen S, Esther, Frans, Gerrit, Gert, Hans K, Hans S, Harry, Herman, Janneke, Joan, Jons, Jos, Joyce, Katarina, Kenneth, Klien, Leon, Lex, Luc, Maarten, Matthijs, Maurice, Milou, Mireille, Rachel, Rene, Richard, Ron, Ronnie, Ruth, Stephan, Paul, en Tom) 
bedanken voor het creëren van een fijne plek! Het plezier van het fietsen wordt immers ook versterkt door een fijne omgeving.

Dat werk en vrije tijd niet gescheiden kunnen worden gezien blijkt uit de band met jou, Sofie. We zijn vier jaar geleden begonnen aan ons traject, en gedurende deze rit zijn we erachter gekomen dat we veel meer gemeen hebben dan alleen onze werkplek. Bedankt dat je mijn paranimf wilt zijn!

Lieve Marije, ik ben heel blij dat ook jij achter mij wilt staan tijdens de verdediging. Het is jammer dat de afstand tussen ons groter is dan in de jaren van ABBA, maar het is een goed gevoel dat er iemand is die er altijd voor me is. Bedankt voor wie je bent!

Alle vrienden en vriendinnen bedankt dat jullie mij regelmatig om me heen lieten kijken tijdens het traject, maar vooral bedankt dat jullie een stukje van je leven met mij willen delen!

Lieve vriendinnetjes, Eeke, Marleen en Hanneke, hoewel we nu echt bijna allemaal weg zijn komen we ook elk jaar een stukje dichterbij... Ik weet zeker dat we over tien jaar weer samen zijn in Maastricht! Hopelijk blijven we in ons leven veel meer delen dan alleen deze route!

Marco, Mariska, Ronald, Ellen en de voorzitter van de Genieters Gerwin, ik vind het heerlijk om met jullie van het leven te kunnen genieten! Hopelijk gaan we nog vaak samen naar de sneeuw, een weekendje weg of gewoon even een wijntje drinken!

Volleybal- en tennis vriendinnen, bedankt voor alle gezellig sport uurtjes maar vooral ook voor de leuke tijd daarnaast. Dat sport gezond is, lijkt me inmiddels wel duidelijk, maar het moet hier worden gezegd dat het ook heel gezellig kan zijn!

Bilbo en Isa, super bedankt voor jullie bijdrage aan het proefschrift! Fijn om wat expertise van jullie te kunnen lenen, het maakte het laatste deel van het proefschrift een stuk gemakkelijker en gezelliger!

Dan mijn schoonfamilie; Johan, Mariëlle, Coent, Sarah, Hanne, René, Cobie, Niels, Juliant, Ruben, Korina, Tjeerd, Elise, Arjen, Rutger, Carolien, Anton, Tom, Kees, Marjet, Ilse, Petra, Elco, Sophie, Adriaan en Jelma. Ik ben heel trots om bij zo'n grote maar vooral warme club te mogen horen! Schoonpap en -mam, ik heb bewondering voor de waardering en respect die jullie voor iedereen hebben! Dank jullie wel! 
De eerste meters die ik kromgebogen over mijn stuur tegen de wind maakte waren 'op de dijk' bij mijn ouders. Pap en mam, ik ben heel dankbaar voor de rustige en stimulerende omgeving die jullie voor me zijn. Bedankt voor de steun die jullie me al mijn hele leven geven!

Lieve Cees, ik vind het geweldig mijn leven met jou te delen! Bedankt dat je het grootse Rotterdam hebt verlaten, om mij deze kans te geven. Ik kijk er al naar uit met jou de toekomst in te rijden! 


\section{CURRICULUM VITAE}

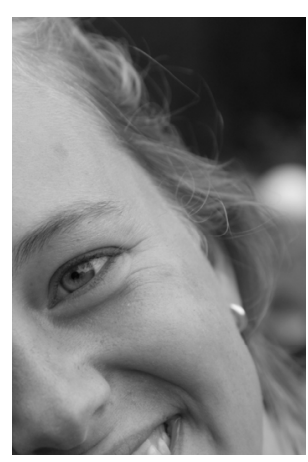

Jantine Slinger werd op 7 mei 1980 geboren in Dirksland. In 1998 behaalde zij het diploma Voorbereidend Wetenschappelijk Onderwijs aan de Rijksscholengemeenschap Goeree Overflakkee te Middelharnis. In aansluiting daarop startte zij met de opleiding Gezondheidswetenschappen aan de Universiteit Maastricht. In 2003 behaalde zij het doctoraaldiploma voor de afstudeerrichtingen Bewegingswetenschappen en Arbeid\&Gezondheid. De studie Arbeid\&Gezondheid werd afgesloten met een onderzoek naar de lange termijn effecten van een bewegingsinterventie bij de gemeente Enschede in opdracht van TNO-Arbeid. De specialisatie Bewegingswetenschappen werd afgesloten met een onderzoek naar de relatie tussen fysieke activiteit en fitheid bij schoolkinderen. In aansluiting op dit stageonderzoek kreeg zij van 2003 tot 2007 een aanstelling als promovenda binnen de vakgroep Bewegingswetenschappen. Het resultaat van het onderzoek dat in deze tijd is uitgevoerd ligt nu voor $u$ in de vorm van dit proefschrift. Naast de aanstelling als promovenda heeft zij in 2006 de master Epidemiologie cum laude afgerond. 


\section{PUBLICATIONS}

Slinger, J.D., Verstappen, F.T., Van Breda, E., Kuipers, H. The effect of body build and BMI on aerobic test performance in school children (10-15 years). J Sports Sci \& Med 2006; 5:699-706.

Slinger, J.D., Van Breda, E., Keizer, H., Rump, P., Hornstra, G., Kuipers, H. Insulin resistance, physical fitness, body composition and leptin concentration in 7-8 year-old children. J Sci Med Sport 2007; in press.

Slinger, J.D., Van Breda, E., Rump, P., Hornstra, G., Kuipers, H. Development of insulin resistance and related variables between 7 and 13 years of age: a longitudinal study. Submitted for publication.

Slinger, J.D., Van Breda, E., Kuipers, H. Recent performance capacity data for Dutch adolescents. Submitted for publication.

Slinger, J.D., Van Breda, E., Brouns, J., Kuipers, H. Effects of a 12 week practice based lifestyle program to counteract overweight in adolescents: an intervention study. Submitted for publication.

\section{Astracts}

Slinger, J., van Breda, E., Kuipers, H. Physical fitness and fasting whole blood glucose concentration in healthy adolescents. AIESEP World Congress. Jyvaskyla, Finland 2006

Slinger, J. 'Body build and aerobic capacity in children'. Fourth European Youth Heart Study Symposium. Odense, Denmark 2006

Slinger, J., Kruisifix, L. Validation of the PAM accelerometer in 11-15 year old children' Objective Measurement of Physical Activity. Odense, Denmark 2006

Slinger, J., van Breda, E., Kuipers, $\mathrm{H}$. Trends in aerobic test performance in Dutch adolescents; the influence of quantity versus quality of fat free mass. International Conference on Physical Activity and Obesity in Children. Toronto, Canada 2007

Slinger, J., van Breda, E., Kuipers, H. Serum leptin but not adiponectin changes during a 12 week community based diet and exercise intervention program. 24th Pediatric Work Physiology Meeting, Talinn, Estonia, 2007 


\section{LIST OF ABBREVIATIONS}

$\begin{array}{ll}\text { ANCOVA } & \text { Analysis of Covariance } \\ \text { BM } & \text { Body Mass } \\ \text { BMI } & \text { Body Mass Index } \\ \text { CBS } & \text { Statistics Netherlands (Centaal Bureau voor Statistiek) } \\ \text { CI } & \text { Confidence Interval } \\ \text { FFM } & \text { Fat Free Mass } \\ \text { FFMI } & \text { Fat Free Mass Index } \\ \text { FM } & \text { Fat Mass } \\ \text { FMI } & \text { Fat Mass Index } \\ \text { HOMA-IR } & \text { Homeostatic Model Assessment for Insulin Resistance } \\ \text { IFG } & \text { Impaired Fasting Glycaemia } \\ \text { M } & \text { Meters } \\ \text { MetS } & \text { Metabolic Syndrome } \\ \text { Min } & \text { Minutes } \\ \text { N } & \text { Aantal } \\ \text { NHANES } & \text { National Health and Nutrition Examination Survey } \\ \text { NNGB } & \text { Healthy Activity Norm (Nationale Norm Gezond Bewegen) } \\ \text { Kg } & \text { Kilogram } \\ \text { Mm } & \text { Milimeter } \\ \text { Type 2 DM } & \text { Type 2 Diabetes Mellitus } \\ \text { OR } & \text { Odds Ratio } \\ \text { P } & \text { P-value } \\ \text { PCOS } & \text { Polycystic Ovary Syndrome } \\ \text { R } & \text { Correlation Coefficient } \\ \text { R } & \text { Explained Variance } \\ \text { VO2max } & \text { Maximal Oxygen Consumption } \\ \text { W } & \text { Watt } \\ \text { Wmax } & \text { Maximal Achieved Wattage } \\ \text { WHO } & \text { World Health Organisation } \\ & \end{array}$


